\title{
Photovoltaics at the mesoscale: insights from quantum-kinetic simulation
}

\author{
Urs Aeberhard \\ IEK-5 Photovoltaik, Forschungszentrum Jülich, 52425 Jülich, Germany \\ E-mail: u.aeberhard@fz-juelich.de
}

\begin{abstract}
This Topical Review discusses insights into the physical mechanisms of nanostructure solar cell operation as provided by numerical device simulation using a state-of-the-art quantum-kinetic framework based on the non-equilibrium Green's function (NEGF) formalism. After a brief introduction to the field of nanostructure photovoltaics and an overview of the existing literature on theoretical description and experimental implementation of such devices, the quantum-kinetic formulation of photovoltaic processes is discussed in detail, together with more conventional modeling approaches such as global detailed balance theory and the semi-classical drift-diffusion-Poisson-Maxwell picture. Application examples provided subsequently include III-V semiconductor nanostructures ranging from ultra-thin absorbers to quantum well and quantum dot solar cell devices. The focus is on common features encountered in photovoltaic nanostructure architectures such as the impact of configurational parameters and operating conditions on device characteristics, and the pronounced deviations from the semiclassical bulk picture. Ultra-thin absorbers are investigated with focus on the effect of built-in fields and contact configuration on radiative rates and currents. For the case of single and multi-quantum-well $p-i-n$ devices, generation, recombination, and escape of carriers are discussed, and quantum well superlattice solar cells are considered with regard to charge carrier transport regimes ranging from band-like transport in miniband states to sequential tunneling between neighboring periods. Double quantum well structures are further studied in the context of tunnel junctions for multijunction solar cell. The investigation of quantum dots covers the fluorescence of colloidal nanoparticles for luminescent solar concentrators as well as the impact of configurational parameters on the photovoltaic properties of regimented quantum dot arrays, both in single-junction and intermediate-band configurations.
\end{abstract}




\section{Introduction}

Ever since the recognition of electricity production from renewable energy sources as a central element of a sustainable society, photovoltaic technology has been in the focus of global research and development efforts. Installed production and manufacturing capacities are increasing exponentially and are approaching the terawatt scale. However, in order to achieve the ambitious goals regarding the reduction in green house gases which is required to keep global warming below $2^{\circ} \mathrm{C}$, a massive replacement of fossil power plants by renewables is required. At the same time, renewable alternatives to photovoltaics (PV) such as biomass or wind energy become less available, either because of competing utilization as food or as resources in chemical industry or due to conflict with landscape and wildlife protection. On the other hand, there is still a huge potential for the installation of PV capacity, especially in the exploitation of existing rooftop area and in the integration into new buildings.

Currently, the market for PV modules is dominated by standard crystalline silicon technology. While this technology is mature and can be scaled to very large capacities owing to the abundance of the raw material, it is energy intensive in the production of the wafers and, with the current record of $26.6 \%$ [1] slowly approaching its theoretical efficiency limit of around $29 \%$ [2]. On the other hand, as the cost of a PV system is no longer dominated by the cost of the solar cell, but by components such as framing, support, wiring, etc. which scale with area, efficiency is also the key to a lower cost of PV technology. This calls for renewed efforts in the research on high-efficiency solar cell concepts, of which a large variety have been proposed over the years, with focus on reducing avoidable thermalization and transmission losses [3-5].

In many cases, the implementation of advanced solar cell architectures demands materials with tailored optoelectronic properties, such as adjustable band gaps in multi-junction architectures [6], reduced thermalization rates in hot carrier solar cells [7] or additional radiative subband transitions in intermediate band concepts [8]. This explains the widespread application of functional nanostructures with configuration-tunable properties in the field of third generation photovoltaics, most prominently in quantum well (QW) [9] and quantum dot (QD) [10] solar cell devices.

Due to the complexity of configuration parame- ter spaces and owing to the cost of nanotechnological fabrication, theoretical modelling and simulation play an important role in the design and optimization of nanostructure-based solar cells. However, the peculiar optoelectronic behavior of low dimensional materials which roots in deviations from bulk physics, questions the validity of the conventional semiclassical bulk picture of the photovoltaic processes if applied to photovoltaics at the mesoscale. On the other hand, while ab initio quantum-mechanical simulations of the nanostructure components provide an accurate description of the electronic and vibrational structure, the exceedingly high computational expense of such methods does not allow (yet) for addressing directly the carrier dynamics to assess local device characteristics. Hence, nanostructure solar cell device simulation conventionally relies on hybrid multiscale simulation approaches that combine quantum-mechanically computed microscopic rates with macroscopic and semiclassical transport equations [11]. In such treatments, while experimental characteristics are reasonably well reproduced by fitting a suitable range of parameters, it is difficult to obtain physical insight into the competition of different dynamical processes, such as, e.g., transport and recombination mediated by localized states, as the processes are not described on equal footing.

In this review, an alternative framework for theory and simulation is discussed, which aims at providing a unified microscopic picture of mesoscopic photovoltaics from the dynamical processes up to device characteristics, while reflecting the microscopic information on key features in the electronic, optical and vibrational structure. The theoretical formalism is based on the non-equilibrium quantum-statistical mechanics of interacting charge carriers, photons and phonons in selectively contacted (open) solid state systems and hence represents the most fundamental and generally valid description of solar cell device operation currently available. As parametrization of the underlying effective model Hamiltonians from first principles is possible, and at the same time, applications to entire solar cell devices with extension beyond the mesoscopic regime are excessively expensive from a computational point of view, an important function of this mesoscale approach will be to bridge and mediate between the pictures at micro- and macroscales in a comprehensive multi-scale simulation framework.

The review is organized as follows. In Sec. 2 the main physical processes governing the photovoltaic 
device operation in nanostructure-based high-efficiency solar cells are discussed in order to define the requirement for corresponding device models. The semiclassical formulation of such models is then introduced in Sec. 3, before the general theory and the numerical implementation of the quantum-kinetic picture is sketched in Sec. 4. In Sec. 5, which forms the main body of the paper, a wide range of applications of the quantum-kinetic theory and simulation framework is provided, from ultra-thin absorbers to quantumwell and quantum-dot solar cells in various device configurations.

\section{Physics of nanostructure-based solar cell device operation}

The main physical processes of photovoltaic device operation are the same at the mesoscale as at the macroscale: photogeneration, transport and extraction at carrier-selective contacts in competition with injection and recombination at finite separation of contact Fermi levels. However, the complex potential landscape in nanostructure-based solar cells gives rise to a number of sub-processes that are not present in bulk devices, as displayed in Fig. 1 for the band profile $\left(E_{\mathrm{C} / \mathrm{V}}\right.$ : conduction/valence band edge) of a prototype quantum photovoltaic device with selective contacts for electrons on the right and holes on the left, respectively. Regarding the generation and recombination processes in (a), in addition to transitions between extended continuum states (1), there are also transitions between confined states (2) or, in some cases, subband transitions (3), while radiative recombination proceeds primarily via the confined nanostructure states below the host continuum (4). (b) Transport processes to consider include carrier capture form continuum to confined states (1), tunneling between localized states (2), phonon-assisted thermal escape from localized to current-carrying extended states (3), and field-assisted direct tunneling escape (4). (c) Electron and hole extraction and injection processes at majority contacts under contact Fermi level splitting $\mu_{\mathrm{n}}-\mu_{\mathrm{p}}=q V_{\text {bias }}$ may be severely affected by the actual potential profile induced by non-ideal band alignment of window and contact passivation layer components. In practice, there are also non-radiative recombination channels associated with nanostructure states, however, the discussion is restricted here to the radiative limit.

\section{The semiclassical standard model of photovoltaic device operation}

While the purpose of this Topical Review is to present a theoretical treatment of nanostructure photovoltaics that reaches beyond the semiclassical bulk picture, it is
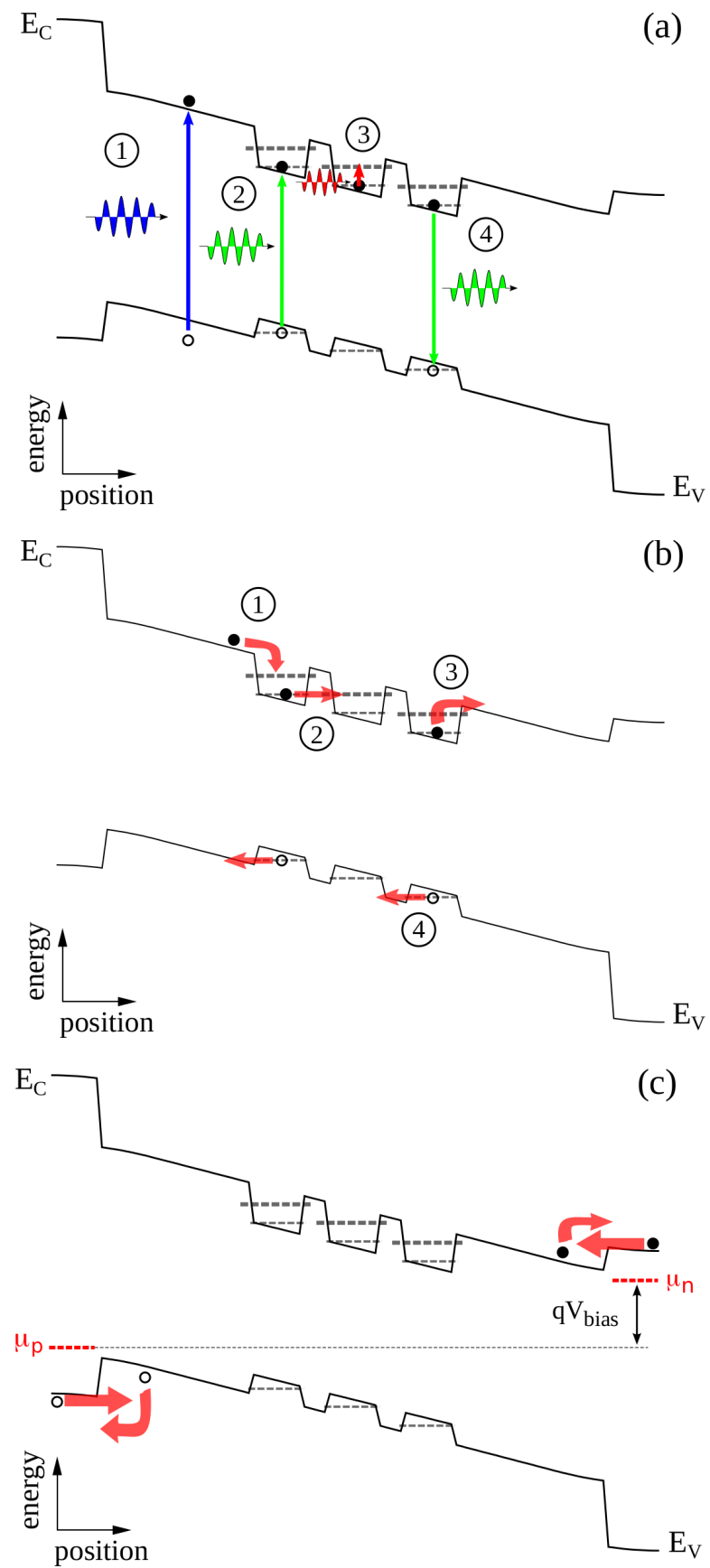

Figure 1. Physical processes governing photovoltaic device operation in prototypical nanostructure solar cell devices: (a) Radiative generation and recombination: (1) generation in the continuum, (2) generation in confined states, (3) radiative subband transition, (4) radiative recombination via confined states. (b) Transport: (1) carrier capture, (2) tunneling between confined states, (3) phonon-assisted thermal escape, (4) escape via field-assisted direct tunneling. (c) Carrier extraction and injection at contacts under a finite splitting of the chemical potentials $\mu_{\mathrm{n}, \mathrm{p}}$ by a voltage $V_{\text {bias }}$.

still instructive to quickly review the latter, as it will be used for validation of the quantum-kinetic treatment in cases where it is valid, and for comparison in 
order to detect deviations in non-bulk-like situations. Furthermore, the semiclassical bulk model keeps its relevance in the context of the multiscale simulation of extended devices, where it is used to describe the bulk components. Among the standard approaches, the two most widely used are considered here, which are, the global detailed balance picture on which most limiting efficiency calculations are based, and the drift-diffusion-Poisson form of the photovoltaic balance equation, which will both be considered in the following.

\subsection{Global detailed balance characteristics}

In the most elementary of the methods, the device characteristics are obtained based on external radiative properties only, by equating the extracted charge current density with the difference of absorbed and emitted photon fluxes [12],

$$
J(V)=-q\left\{\Phi_{\mathrm{abs}}\left(\phi_{0 \gamma}^{\swarrow}\right)-\Phi_{\mathrm{em}}(V)\right\},
$$

where $q$ is the elementary charge, $\phi_{0 \gamma}^{\swarrow}$ is the incident photon flux and $V$ is the voltage between contacts. In the global radiative detailed balance limit, the two quantities are related via their dependence on the absorptance of the device $[12,13]$,

$$
\begin{aligned}
\Phi_{\mathrm{abs}}\left(\phi_{0 \gamma}^{\swarrow}\right)= & \int d E_{\gamma} \phi_{0 \gamma}^{\swarrow}\left(E_{\gamma}\right) a\left(\Omega^{\swarrow}, E_{\gamma}\right), \\
\Phi_{\mathrm{em}}(V) \approx & \int d E_{\gamma} \int d \Omega a\left(\Omega, E_{\gamma}\right) \bar{\phi}_{\mathrm{bb}}\left(\Omega, E_{\gamma}\right) \\
& \times\left\{\exp \left(q V / k_{\mathrm{B}} T\right)-1\right\},
\end{aligned}
$$

where $E_{\gamma}$ is the photon energy, $a$ is the absorptance (at angle of incidence $\Omega^{\swarrow}$ ) - related to the absorption coefficient $\alpha$ and refractive index $n$ via some optical model for the light propagation, such as, e.g., LambertBeers law or the Transfer-Matrix Method (TMM) for coherent wave propagation - and $\bar{\phi}_{\mathrm{bb}}$ is the black-body radiation flux for emission into solid angle $\Omega$. In the above formulation, the model assumes perfect carrier extraction due to infinite mobility, corresponding to flat quasi-Fermi levels split by the voltage at the contacts, $\Delta \mu=q V$. The model can be extended to the case of finite mobility and non-radiative recombination by replacing in Eq. (3) the absorptance with the external quantum efficiency [14]. However, there are limits in the applicability of this reciprocity relation that stem from the voltage or illumination dependence of the external quantum efficiency which lead to the breakdown of the superposition principle [15].

\subsection{Semiclassical balance equations with local Fermi-Golden-Rule rates}

In order to capture effects of finite mobility, the transport of photogenerated or electronically injected charge carriers needs to be considered. For a detailed investigation of the impact of a specific nanostructured device component on the overall device characteristics, the local charge carrier dynamics has to be included in the model. In general, this is achieved by combining a drift-diffusion model for charge transport (upper/lower sign is for electrons/holes)

$$
\begin{gathered}
\mathbf{J}_{c}(\mathbf{r})=\mp q\left\{ \pm \rho_{c}(\mathbf{r}) \mu_{c}(\mathbf{r}) \nabla \varphi(\mathbf{r})-D_{c}(\mathbf{r}) \nabla \rho_{c}(\mathbf{r})\right\} \\
(c=e, h)
\end{gathered}
$$

with Fermi-Golden-Rule rates for the local carrier generation $(\mathcal{G})$ and recombination $(\mathcal{R})$

$$
\begin{aligned}
\mathcal{G}(\mathbf{r}) & =\int d E_{\gamma} \eta_{\text {gen }}\left(E_{\gamma}\right) \alpha\left(\mathbf{r}, E_{\gamma}\right) \phi_{\gamma}\left(\mathbf{r}, E_{\gamma}\right), \\
\mathcal{R}(\mathbf{r}) & =\mathcal{B}(\mathbf{r}) \rho_{e}(\mathbf{r}) \rho_{h}(\mathbf{r}), \\
\mathcal{B}(\mathbf{r}) & =n_{i}^{-2} \int d E_{\gamma} \alpha\left(\mathbf{r}, E_{\gamma}\right) \tilde{\phi}_{\mathrm{bb}}\left(E_{\gamma}\right),
\end{aligned}
$$

obtained from the local microscopic electronic structure information, in carrier-specific balance equations for charge continuity

$$
\mp q^{-1} \nabla \cdot \mathbf{J}_{c}(\mathbf{r})=\mathcal{G}(\mathbf{r})-\mathcal{R}(\mathbf{r}), \quad(c=e, h)
$$

coupled to the Poisson equation for the electrostatic potential $\varphi$,

$$
\epsilon_{0} \nabla \cdot\{\varepsilon(\mathbf{r}) \nabla \varphi(\mathbf{r})\}=q\left\{\rho_{e}(\mathbf{r})-\rho_{h}(\mathbf{r})-N_{\mathrm{dop}}(\mathbf{r})\right\} .
$$

In the above equations, $\mu$ is the mobility, $D$ the diffusion constant, $\rho\left(n_{i}\right)$ the (intrinsic) carrier density, $\phi_{\gamma}$ is the local photon flux due to the external illumination, $\eta_{\text {gen }}$ is the fraction of photons generating electron-hole pairs, $\tilde{\phi}_{\mathrm{bb}}[n]$ is the angleintegrated black-body flux for isotropic emission into medium with refractive index $n, \alpha$ is the absorption coefficient, $\tau$ is the carrier lifetime associated with the recombination process, $\varepsilon_{0}$ and $\varepsilon$ are the free space and relative permittivities, and $N_{\mathrm{dop}} \equiv N_{\mathrm{D}}^{+}-N_{\mathrm{A}}^{-}$is the density of ionized dopants. Conventionally, the carrier density is expressed in terms of an effective density of states $\mathcal{N}$ that reflects the electronic structure close to the band edge, and of the carrier distribution function, for which it is common to use Boltzmann statistics with quasi-Fermi level $E_{\mathrm{F}_{c}}(c=e, h)$ :

$$
\rho_{c}(\mathbf{r})=\mathcal{N}_{c}(\mathbf{r}) \exp \left\{\left[ \pm E_{\mathrm{F}_{c}}(\mathbf{r}) \mp E_{\mathrm{B}}(\mathbf{r})\right] / k_{\mathrm{B}} T\right\}
$$

where upper (lower) sign applies to electrons (holes), and $E_{\mathrm{B}}$ is the band edge energy. Solution of Eqns. (4), (8)-(10) provides the current-voltage characteristics as a function of quasi-Fermi levels $E_{\mathrm{F}_{e, h}}$ for electrons and holes and of the electrostatic potential $\varphi$. Modified versions of Expr. (8) have been used in cases with 
vanishing current between absorbers and/or absent coupling to contact states $[16,17]$. Such a hybrid approach is limited in validity by the assumptions underlying the drift-diffusion picture, i.e., bandlike transport with completely thermalized carrier distributions, which does not include any quantum effects such as confinement, tunneling or ballistic transport on very short length scales. Moreover, in most cases, the model used for the electronic structure relies on the flat band bulk picture, which is not applicable in nanostructure regions.

\section{Non-equilibrium quantum statistical mechanics formulation of photovoltaic processes}

The challenges of describing photovoltaic device operation under consideration of quantum effects are manifold. Firstly, one has to treat an open quantum system, which in principle requires a description based on scattering states rather than the eigenstates provided by the solution of Schrödinger's equation for the closed system. Due to the essential inclusion of light-matter interaction and the sizable effects of electron-phonon coupling under the standard condition of room temperature operation, a mixed state representation is indicated. Among the suitable theories, the non-equilibrium Greens function (NEGF) formalism is most versatile and powerful and has found wide-spread application in the modeling of nanostructure-based quantum opto-electronic devices such as photodetectors based on QW [18] and QD [19], QW lasers [20], quantum cascade lasers [21, 22], and QW LEDs [23]. In the field of nanostructure photovoltaics, applications of the NEGF formalism so far include carbon nanotube photodiodes [24], multiQW and QW superlattice solar cells [25-27], nanowire solar cells [28], QD superlattice solar cells [29, 30], ultra-thin absorber devices [31,32], and QW tunnel junctions for multi-junction solar cells [33].

Powerful and versatile as it is, the NEGF formalism has its foundations in advanced concepts of many-body and quantum-field theories which are not widely used in photovoltaics. For this reason, while trying to remain self-contained, the discussion of the general theory is limited here to what is relevant for the examples in the application section. For a more detailed and complete presentation of the NEGF theory of quantum photovoltaic devices, the reader is referred to Ref. [34].

\subsection{Theoretical formalism}

In contrast to the semiclassical picture, the quantumstatistical mechanics description does not aim to match complex reality by adjusting a range of parameters of a simplified model. Instead, a simplified physical universe containing the relevant degrees freedom and interactions is constructed and subjected to the external conditions of device operation, from which the physical device behaviour emerges in its full complexity. Hence, while the basic structure of the material and its inherent interactions are modeled on the basis of microscopic information, the device characteristics are produced by the basic physical laws prevailing in the model universe.

4.1.1. Hamiltonian Mathematically, the microscopic model universe relevant for photovoltaic device simulation is represented by the model Hamiltonian for a system of interacting electrons, photons and phonons:

$$
\begin{aligned}
\hat{H} & =\hat{H}_{e}+\hat{H}_{p}+\hat{H}_{\gamma}+\hat{H}_{I}, \\
\hat{H}_{I} & =\hat{H}_{e \gamma}+\hat{H}_{e p}+\hat{H}_{p \gamma},
\end{aligned}
$$

where $\hat{H}_{I}$ describes the mutual interactions of charge carriers, photons and phonons. The electronic part $\hat{H}_{e}=\hat{H}_{e 0}+\hat{H}_{e e}$ contains the non-interacting part $\hat{H}_{e 0}$ and the electron-electron interaction part $\hat{H}_{e e}$. The non-interacting term provides the kinetic energy and contains the potential for the interaction of valence electrons with the ion cores, and in most practical implementations includes also the Hartree term of the Coulomb interaction corresponding to the solution of Poisson's equation that considers carrier-carrier interactions on a mean-field level. Similarly, $\hat{H}_{p}=$ $\hat{H}_{p 0}+\hat{H}_{p p}$, where the first term contains the kinetic energy of the lattice and the second term encodes the phonon-phonon interaction that is relevant, e.g., for anharmonic decay of vibrational modes [35]. The photon term, on the other hand, contains only the freefield contribution $H_{\gamma 0}$.

In the quantum-statistical mechanics picture used here, the quantized electronic, vibrational and optical degrees of freedom are expressed in terms of the corresponding field operators, namely, the fermion field operator $\hat{\Psi}$ for the charge carriers, the quantized vector potential $\hat{\mathbf{A}}$ for the transverse photons and the ionic lattice displacement field $\hat{\mathcal{U}}$ for the phonons. On the one hand, the bosonic fields enter directly in the elements of the interaction Hamiltonian $\hat{H}_{I}$. The electron-photon Hamiltonian for minimal coupling and using Coulomb gauge is given by

$$
\hat{H}_{e \gamma}(\mathbf{r}, t)=\frac{e}{m_{0}} \hat{\mathbf{A}}(\mathbf{r}, t) \cdot \hat{\mathbf{p}}
$$

where $\hat{\mathbf{p}} \equiv-i \hbar \nabla_{\mathbf{r}}$ is the momentum operator. The electron-phonon term is expressed as

$$
\hat{H}_{e p}(\mathbf{r}, t)=\sum_{\mathbf{L}, \boldsymbol{\kappa}} \hat{\mathcal{U}}(\mathbf{L}+\boldsymbol{\kappa}, t) \cdot \nabla V_{e i}[\mathbf{r}-(\mathbf{L}+\boldsymbol{\kappa})],
$$


where $V_{e i}$ denotes the electron-ion potential, $\mathbf{L}$ is the equilibrium lattice vector and $\boldsymbol{\kappa}$ the basis vector within the unit cell [38]. Direct interaction of light with the lattice $\left(\hat{H}_{p \gamma}\right)$ is not considered here.

4.1.2. Non-equilibrium Green's functions On the other hand, the field operators define the single-particle Green's functions (GF) $\ddagger$

$$
\begin{aligned}
G(\underline{1}, \underline{2})= & -\frac{i}{\hbar}\left\langle\hat{\Psi}(\underline{1}) \hat{\Psi}^{\dagger}(\underline{2})\right\rangle_{\mathcal{C}}, & & \text { (electrons) } \\
\mathcal{D}_{\mu \nu}^{\gamma}(\underline{1}, \underline{2})= & -\frac{i}{\hbar} \frac{1}{\mu_{0}}\left[\left\langle\hat{A}_{\mu}(\underline{1}) \hat{A}_{\nu}(\underline{2})\right\rangle_{\mathcal{C}}\right. & & \\
& \left.-\left\langle\hat{A}_{\mu}(\underline{1})\right\rangle_{\mathcal{C}}\left\langle\hat{A}_{\nu}(\underline{2})\right\rangle_{\mathcal{C}}\right], & & \text { (photons) }(16) \\
\mathcal{D}_{\alpha \beta}^{p}(\underline{\tilde{1}}, \underline{\tilde{2}})= & -\frac{i}{\hbar}\left\langle\hat{\mathcal{U}}_{\alpha}(\underline{\tilde{1}}) \hat{\mathcal{U}}_{\beta}(\underline{\tilde{2}})\right\rangle_{\mathcal{C}}, & & \text { (phonons) }(17)
\end{aligned}
$$

where $\mu, \nu$ and $\alpha, \beta$ are cartesian indices and $\langle\ldots\rangle_{C}$ denotes the contour-ordered operator average peculiar to non-equilibrium quantum statistical mechanics [36, 37] for arguments $\underline{1}=\left(\mathbf{r}_{1}, \underline{\mathrm{t}}_{1}\right)$ with $\underline{\mathrm{t}}_{1}$ on the Keldysh contour [37]. The photon GF describes the fluctuations with respect to the macroscopic average responsible for spontaneous emission. In the case of the lattice displacement field, the continuous spatial coordinate $\mathbf{r}$ is replaced by the discrete ion position vectors $\mathbf{R}=$ $\mathbf{L}+\boldsymbol{\kappa} \equiv \mathbf{L} \boldsymbol{\kappa}$. The associated four-vector is $\tilde{1} \equiv$ $\left(\mathbf{L}_{1} \boldsymbol{\kappa}_{1}, \underline{\mathrm{t}}_{1}\right)$. The GFs are determined as the solutions of corresponding Dyson's equations [20,38,39],

$$
\int_{\mathcal{C}} d \underline{3}\left[G_{0}^{-1}(\underline{1}, \underline{3})-\Sigma(\underline{1}, \underline{3})\right] G(\underline{3}, \underline{2})=\delta(\underline{1}-\underline{2}),
$$

$$
\int_{\mathcal{C}} d \underline{3}\left[\left(\overleftrightarrow{\mathcal{D}}_{0}^{\gamma}\right)^{-1}(\underline{1}, \underline{3})-\overleftrightarrow{\boldsymbol{\Pi}}^{\gamma}(\underline{1}, \underline{3})\right] \overleftrightarrow{\mathcal{D}}^{\gamma}(\underline{3}, \underline{2})=\overleftrightarrow{\boldsymbol{\delta}}(\underline{1}-\underline{2})
$$

$$
\int_{\mathcal{C}} d \underline{3}\left[\left(\mathcal{D}_{0}^{p}\right)^{-1}(\underline{\tilde{1}}, \underline{\tilde{3}})-\boldsymbol{\Pi}^{p}(\underline{\tilde{1}}, \underline{\tilde{3}})\right] \mathcal{D}^{p}(\underline{\tilde{3}}, \underline{\tilde{2}})=\boldsymbol{\delta}(\underline{\tilde{1}}-\underline{\tilde{2}}),
$$

where the integration is $\int_{\mathcal{C}} d \underline{1} \equiv \int_{\mathcal{C}} d \underline{\mathrm{t}}_{1} \int d^{3} r_{1}$. The GFs $G_{0}, \mathcal{D}_{0}^{\gamma}$ and $\mathcal{D}_{0}^{p}$ are the propagators for non-interacting electrons, photons and phonons, respectively, $\leftrightarrow$ denotes transverse degrees of freedom and boldface expresses tensorial quantities. The electronic selfenergy $\Sigma$ encodes the renormalization of the charge carrier GFs due to the interactions with photons and phonons and other carriers, and enables thus the description of charge carrier generation, recombination and relaxation processes. It is responsible for the appearance of excitonic effects in the carrier spectrum and leads to band-gap renormalization

$\ddagger$ The spin of charge carriers is not treated explicitly here - where required, a factor of two is added to account for summation over spin, while in general, a spinless representation is assumed. under high excitation [40]. Charge injection and extraction at contacts is considered via an additional boundary self-energy term reflecting the openness of the system. The photon and phonon self-energy tensors $\overleftrightarrow{\boldsymbol{\Pi}}^{\gamma}$ and $\boldsymbol{\Pi}^{p}$ describe the renormalization of the optical and vibrational excitation modes due to the interaction with the electronic system, i.e. absorption and emission of photons and phonons, leading to phenomena such as photon recycling or hot carrier effects and including excitonic signatures in the bosonic spectra.

4.1.3. Interaction self-energies Formally, the selfenergies in the above equations follow from the expansion of the Green's functions in terms of the perturbation interactions that govern the propagation of the system on the complex time contour $\S$. In the case of the electronic GF, this corresponds to the expansion of the exponential term in

$$
G\left(\mathbf{r}, t ; \mathbf{r}^{\prime}, t^{\prime}\right)=-\frac{i}{\hbar}\left\langle e^{-\frac{i}{\hbar} \int_{C} d s \hat{\mathcal{H}}_{I}(s)} \hat{\Psi}(\mathbf{r}, t) \hat{\Psi}^{\dagger}\left(\mathbf{r}^{\prime}, t^{\prime}\right)\right\rangle_{C},
$$

with respect to

$$
\hat{\mathcal{H}}_{I}(t)=\int d^{3} r \hat{\Psi}^{\dagger}(\mathbf{r}, t) \hat{H}_{I} \hat{\Psi}(\mathbf{r}, t)
$$

which allows for the identification of the self-energy term in

$G(\underline{1}, \underline{2})=G_{0}(\underline{1}, \underline{2})+\int_{C} d \underline{3} \int_{C} d \underline{4} G_{0}(\underline{1}, \underline{3}) \Sigma(\underline{3}, \underline{4}) G(\underline{4}, \underline{2})$.

To first order in the interaction, finite contributions are obtained only for fields with macroscopic average, as in the case of the coupling to classical external illumination, which provides the instantaneous selfenergy for stimulated processes [42]:

$$
\Sigma_{e \gamma}^{\delta}(\underline{1})=\frac{e}{m_{0}}\langle\hat{\mathbf{A}}(\underline{1})\rangle_{\mathcal{C}} \cdot \hat{\mathbf{p}}(1) \equiv \frac{e}{m_{0}} \overline{\mathbf{A}}(\underline{1}) \cdot \hat{\mathbf{p}}(1),
$$

where $\overline{\mathbf{A}}$ is the classical vector potential of the external illumination. As this singular self-energy induces offdiagonal terms with respect to band indices, a band decoupling procedure is applied in order to arrive at a diagonal self-energy [42,43]: \|

$$
\Sigma^{\alpha}\left(\underline{1}, \underline{1}^{\prime}\right)=\Sigma^{\delta}(\underline{1}) G^{\alpha}\left(\underline{1}, \underline{1}^{\prime}\right) \Sigma^{\delta}\left(\underline{1}^{\prime}\right), \quad \alpha=R, A, \lessgtr,
$$

$\S$ Alternatively, they can be derived using functional derivatives, as, e.g., in Ref. [41]

$\|$ The GF in (25) do not consider the effects of interband coupling; however, as the occupation of electrons in the valence band and of holes in the conduction band are only marginally modified under the standard conditions of photovoltaic device operation (i.e., low illumination intensity), it is usually safe to use the full GFs. 
where GF and self-energy are associated with different bands coupled by the singular interaction terms. To second order in the interaction, on the level of the self-consistent Born approximation (SCBA), the self-energies for electron-photon and electron-phonon interaction are obtained in the following general form corresponding to the Fock terms of the diagrammatic expansion:

$$
\begin{aligned}
\Sigma_{e \gamma}(\underline{1}, \underline{2})= & i \hbar \mu_{0}\left(\frac{e}{m_{0}}\right)^{2} \sum_{\alpha, \beta} \hat{p}_{\alpha}\left(1,1^{\prime}\right) G(\underline{1}, \underline{2}) \\
& \times\left.\hat{p}_{\beta}(2) \mathcal{D}_{\alpha \beta}^{\gamma}\left(\underline{2}, \underline{1^{\prime}}\right)\right|_{1^{\prime}=1},
\end{aligned}
$$

for the electron-photon self-energy $[20,40]$, where $\mu_{0}$ is the vacuum permeability and

$$
\hat{p}_{\alpha}\left(1,1^{\prime}\right) \equiv\left\{\hat{p}_{\alpha}(1)-\hat{p}_{\alpha}\left(1^{\prime}\right)\right\} / 2,
$$

and

$$
\begin{aligned}
\Sigma_{e p}(\underline{1}, \underline{2})= & i \hbar \sum_{\mathbf{L}, \boldsymbol{\kappa}} \sum_{\mathbf{L}^{\prime}, \boldsymbol{\kappa}^{\prime}} \sum_{\alpha, \beta} \mathcal{F}_{\alpha}\left(\mathbf{r}_{1}-\mathbf{L} \boldsymbol{\kappa}\right) \mathcal{F}_{\beta}\left(\mathbf{r}_{2}-\mathbf{L}^{\prime} \boldsymbol{\kappa}^{\prime}\right) \\
& \times \mathcal{D}_{\alpha \beta}^{p}\left(\mathbf{L} \boldsymbol{\kappa}, \underline{\mathrm{t}}_{1} ; \mathbf{L}^{\prime} \boldsymbol{\kappa}^{\prime}, \underline{\mathrm{t}}_{2}\right) G(\underline{1}, \underline{2}),
\end{aligned}
$$

for the electron-phonon self-energy [38,44], with $\mathcal{F}=$ $\nabla V_{e i}$. Similarly, the random phase approximation of the photon self-energy is obtained in terms of electronic Green's functions and momentum operator elements as follows $[20,40]: \uparrow$

$$
\begin{aligned}
\Pi_{\alpha \beta}(\underline{1}, \underline{2})= & -2 i \hbar \mu_{0}\left(\frac{e}{m_{0}}\right)^{2} \hat{p}_{\alpha}\left(1,1^{\prime}\right) G(\underline{1}, \underline{2}) \\
& \times\left.\hat{p}_{\beta}(2) G\left(\underline{2}, \underline{1}^{\prime}\right)\right|_{1^{\prime}=1},
\end{aligned}
$$

A discussion of the phonon self-energy as used for the description of anharmonic decay can be found in Ref. [35].

4.1.4. Contacts It was shown in Sec. 2 that extraction and injection of charge carriers at (selective) contacts count among the fundamental processes of photovoltaic device operation. In order to describe these processes in the present microscopic picture, the treatment of contacts in the NEGF theory of quantum transport is adopted [45-47]. There, contacts are described as reservoirs with an equilibrium population of carriers characterized by a fixed chemical potential. In the treatment of the complete system consisting of device region and reservoirs, the effect of the latter is condensed into respective self-energies that describe the renormalization of the device state and the lifetime broadening induced by coupling to the contacts. In this way, the physical extension of the contact region that needs to be consider explicitly can be reduced to the

9 There is a factor of two for spin. zones of actual coupling. Conventionally, the resulting self-energy is expressed as

$$
\Sigma^{B}=\tau^{\dagger} g^{B} \tau
$$

where $\tau$ encodes the elements of the coupling Hamiltonian between contact and device, and $g^{B}$ is the surface Green's function of the semi-infinite reservoir. The problem of the coupling to reservoirs then reduces to the calculation of the contact $\mathrm{GF} g^{B}$, which can be achieved via surface GF methods using decimation techniques $[48,49]$, conformal maps [50] or complex band methods [51-54]. Similar surface GF approaches can also be used for photons $[41,55]$ or phonons $[35,56,57]$ in systems that are open in the optical or vibrational sense, respectively.

4.1.5. Steady-state device characteristics The nonequilibrium Green's functions provide any kind of physical information on the electronic, optical and vibrational properties of the system that can be expressed via single particle operators. However, before any characteristics can be computed, the Green's functions first have to be determined in the physical space of real time arguments. For this purpose, equations (18) - (20) and the selfenergy expressions are reformulated in terms of contour-ordered quantities via Green's functions that are labeled according to the sequence of their time arguments on the contour, which introduces the labels " $<$ " and " $>$ " for components that are ordered chronologically or anti-chronologically, but with different sign of the imaginary part of the time arguments, in addition to chronological and antichronological components ("Keldysh formalism") [37]. The latter are usually replaced by the more familiar retarded ("R") and advanced ("A") components. The transformation to real-time functions and integrations can be facilitated by following the so-called Langreth rules [58].

For investigations focused on steady-state operation, the time arguments are replaced by the difference $t-t^{\prime}$, which is then Fourier-transformed to energy space. In the case of the electron density, for instance, this yields

$$
\begin{aligned}
n(\mathbf{r}, t) & =-\lim _{\mathbf{r}^{\prime} \rightarrow \mathbf{r}} \lim _{t^{\prime} \rightarrow t^{+}} i \hbar \operatorname{tr}\left\{G^{<}\left(\mathbf{r}, t ; \mathbf{r}^{\prime}, t^{\prime}\right)\right\} \\
\rightarrow n(\mathbf{r}) & =-\lim _{\mathbf{r}^{\prime} \rightarrow \mathbf{r}} \lim _{t^{\prime} \rightarrow t^{+}} i \hbar \operatorname{tr}\left\{G^{<}\left(\mathbf{r}, \mathbf{r}^{\prime} ; t-t^{\prime}\right)\right\} \\
& =-\lim _{\mathbf{r}^{\prime} \rightarrow \mathbf{r}} \lim _{t^{\prime} \rightarrow t^{+}} i \int \frac{d E}{2 \pi} e^{\frac{i}{\hbar} E\left(t-t^{\prime}\right)} \operatorname{tr}\left\{G^{<}\left(\mathbf{r}, \mathbf{r}^{\prime} ; E\right)\right\} \\
& =-i \int \frac{d E}{2 \pi} \operatorname{tr}\left\{G^{<}(\mathbf{r}, \mathbf{r} ; E)\right\}
\end{aligned}
$$

where the trace is over spin, orbital indices etc. Similarly, the steady-state electron current acquires the 
form

$\mathbf{j}_{e}(\mathbf{r})=-\lim _{\mathbf{r}^{\prime} \rightarrow \mathbf{r}} \frac{\hbar}{2 m_{0}}\left(\nabla_{\mathbf{r}}-\nabla_{\mathbf{r}^{\prime}}\right) \int \frac{d E}{2 \pi} \operatorname{tr}\left\{G^{<}\left(\mathbf{r}, \mathbf{r}^{\prime} ; E\right)\right\}$.

Expressions (34) and (35) also define spectral densities and currents in terms of the Green's functions. Another spectral quantity of interest is the local density of states (LDOS) determined by the retarded component via

$$
\mathscr{D}(\mathbf{r}, E)=-\frac{1}{\pi} \operatorname{tr}\left\{\Im G^{R}(\mathbf{r}, \mathbf{r} ; E)\right\} .
$$

The microscopic counterpart of the net (volume) rate term in the steady-state continuity equation for charge carriers - Eq. (8) - which is at the heart of the semiclassical theory of photovoltaic device operation is obtained upon application of the divergence operator on (35):

$$
\begin{aligned}
& \mathcal{R}_{\text {tot }}^{\mathrm{el}}(\mathbf{r})=-\int \frac{d E}{2 \pi \hbar} \int d^{3} r^{\prime} \operatorname{tr}\left[\Sigma^{R}\left(\mathbf{r}, \mathbf{r}^{\prime} ; E\right) G^{<}\left(\mathbf{r}^{\prime}, \mathbf{r} ; E\right)\right. \\
& +\Sigma^{<}\left(\mathbf{r}, \mathbf{r}^{\prime} ; E\right) G^{A}\left(\mathbf{r}^{\prime}, \mathbf{r} ; E\right)-G^{R}\left(\mathbf{r}, \mathbf{r}^{\prime} ; E\right) \Sigma^{<}\left(\mathbf{r}^{\prime}, \mathbf{r} ; E\right) \\
& \left.-G^{<}\left(\mathbf{r}, \mathbf{r}^{\prime} ; E\right) \Sigma^{A}\left(\mathbf{r}^{\prime}, \mathbf{r} ; E\right)\right] .
\end{aligned}
$$

The integrand defines again the net energy-resolved local scattering rate. If the energy range of integration is restricted, only processes that connect energies within that window cancel upon integration, while processes with initial or final energies outside the window provide finite contributions to the net rate. This is the case for instance in optical interband transitions, if the rate is evaluated for a specific carrier species. The steady-state Green's function components in the above expressions are obtained from the corresponding Dyson and Keldysh equations resulting from the transformed expressions (18)-(20):

$$
\begin{aligned}
& G^{R(A)}\left(\mathbf{r}, \mathbf{r}^{\prime} ; E\right)=G_{0}^{R(A)}\left(\mathbf{r}, \mathbf{r}^{\prime} ; E\right) \\
&+\int d^{3} r_{1} \int d^{3} r_{2}\left[G_{0}^{R(A)}\left(\mathbf{r}, \mathbf{r}_{1} ; E\right)\right. \\
&\left.\times \Sigma^{R(A)}\left(\mathbf{r}_{1}, \mathbf{r}_{2} ; E\right) G^{R(A)}\left(\mathbf{r}_{2}, \mathbf{r}^{\prime} ; E\right)\right], \\
& G^{\lessgtr}\left(\mathbf{r}, \mathbf{r}^{\prime} ; E\right)= \int d^{3} r_{1} \int d^{3} r_{2}\left[G^{R}\left(\mathbf{r}, \mathbf{r}_{1} ; E\right)\right. \\
&\left.\times \Sigma^{\lessgtr}\left(\mathbf{r}_{1}, \mathbf{r}_{2} ; E\right) G^{A}\left(\mathbf{r}_{2}, \mathbf{r}^{\prime} ; E\right)\right] .
\end{aligned}
$$

The representation in energy space of the contact or boundary self-energy components in the above equations reflect the density of states and occupation of the reservoirs:

$$
\begin{aligned}
\Sigma^{\mathrm{B},<}(\cdot ; E) & =i f\left(\mu_{\mathrm{B}}, E\right) \Gamma^{\mathrm{B}}(\cdot ; E), \\
\Sigma^{\mathrm{B},>}(\cdot ; E) & =-i\left[1-f\left(\mu_{\mathrm{B}}, E\right)\right] \Gamma^{\mathrm{B}}(\cdot ; E), \\
\Gamma^{\mathrm{B}}(\cdot ; E) & \equiv i\left[\Sigma^{\mathrm{B}, R}(\cdot ; E)-\Sigma^{\mathrm{B}, A}(\cdot ; E)\right] \\
& =i\left[\Sigma^{\mathrm{B},>}(\cdot ; E)-\Sigma^{\mathrm{B},<}(\cdot ; E)\right],
\end{aligned}
$$

where $f\left(\mu_{\mathrm{B}}, E\right)$ is the Fermi-Dirac distribution for chemical potential $\mu_{\mathrm{B}}$, and the contact broadening function $\Gamma^{\mathrm{B}}$ is a measure for the life-time reduction due to coupling to the contact. The real part of the retarded self-energy, on the other hand, describes an energy shift induced in the device states. These two effects - energy shift and broadening - are characteristic for any self-energy. The interaction self-energies in equations (39) are

$$
\begin{array}{r}
\sum_{e \gamma}^{\lessgtr(\mathrm{coh})}\left(\mathbf{r}, \mathbf{r}^{\prime} ; E\right)=\left(\frac{e}{m_{0}}\right)^{2} \sum_{\mu \nu} A_{\mu}\left(\mathbf{r}, E_{\gamma}\right) p^{\mu}(\mathbf{r}) \\
\times G^{\lessgtr}\left(\mathbf{r}, \mathbf{r}^{\prime} ; E \mp E_{\gamma}\right) A_{\nu}^{*}\left(\mathbf{r}^{\prime} ; E_{\gamma}\right) p^{\nu *}\left(\mathbf{r}^{\prime}\right),
\end{array}
$$

for the coherent coupling to the incident radiation at energy $E_{\gamma}$, and

$$
\begin{aligned}
& \sum_{e \gamma}^{\lessgtr(\text { inc })}\left(\mathbf{r}, \mathbf{r}^{\prime} ; E\right)=i \hbar \mu_{0}\left(\frac{e}{m_{0}}\right)^{2} \sum_{\mu \nu} \lim _{\mathbf{r}^{\prime \prime} \rightarrow \mathbf{r}} \frac{1}{2}\left\{\hat{p}^{\mu}(\mathbf{r})\right. \\
& \left.\quad-\hat{p}^{\mu}\left(\mathbf{r}^{\prime \prime}\right)\right\} \lim _{\mathbf{r}^{\prime \prime \prime} \rightarrow \mathbf{r}^{\prime}} \hat{p}^{\nu}\left(\mathbf{r}^{\prime \prime \prime}\right) \int \frac{d E^{\prime}}{2 \pi \hbar}\left[\mathcal{D}_{\mu \nu}^{\gamma, \lessgtr}\left(\mathbf{r}^{\prime \prime}, \mathbf{r}^{\prime \prime \prime} ; E^{\prime}\right)\right. \\
& \left.\quad \times G\left(\mathbf{r}, \mathbf{r}^{\prime} ; E-E^{\prime}\right)\right]
\end{aligned}
$$

for incoherent coupling to electromagnetic field fluctuations. Similarly, the self-energy for coupling to phonons reads

$$
\begin{aligned}
\Sigma_{e p}^{\lessgtr}\left(\mathbf{r}, \mathbf{r}^{\prime} ; E\right) & =i \hbar \sum_{\mathbf{L}, \boldsymbol{\kappa}} \sum_{\mathbf{L}^{\prime}, \boldsymbol{\kappa}^{\prime}} \sum_{\alpha, \beta} \mathcal{F}_{\alpha}(\mathbf{r}-\mathbf{L} \boldsymbol{\kappa}) \\
& \times \mathcal{F}_{\beta}\left(\mathbf{r}^{\prime}-\mathbf{L}^{\prime} \boldsymbol{\kappa}^{\prime}\right) \int \frac{d E^{\prime}}{2 \pi \hbar}\left[\mathcal{D}_{\beta \alpha}^{p, \lessgtr}\left(\mathbf{L} \boldsymbol{\kappa}, \mathbf{L}^{\prime} \boldsymbol{\kappa}^{\prime} ; E^{\prime}\right)\right. \\
& \left.\times G^{\lessgtr}\left(\mathbf{r}, \mathbf{r}^{\prime} ; E-E^{\prime}\right)\right] .
\end{aligned}
$$

For the weak coupling considered here, the renormalization of the quasiparticle energies due to the real part of the retarded self-energy component can be neglected, and the retarded component can be approximated by $\Sigma^{R}=\frac{1}{2}\left(\Sigma^{>}-\Sigma^{<}\right)$.

Expressions (45) and (46) relate the electronic quantities to optical and vibrational properties by explicit dependence on the respective Green's functions. While this relation is given here in its most general form, its is often possible to use simplified treatments for either the optical or the vibrational properties, e.g., in cases where these degrees of freedom behave bulklike and/or remain at equilibrium. This is commonly the case for the phonon properties, which will thus not be treated beyond the level of an equilibrium bulk propagator. For the photons, on the other hand, explicit consideration of photon confinement effects is relevant for any kind of optically thin solar cell device, which affects most of the high-efficiency architectures discussed here. Hence, a first step in the direction of a unified quantum theory of photovoltaics at the 
nanoscale consists in the consideration of electrons and photons with the same degree of rigor.

In effect, similar expressions for physical characteristics can be derived for photonic quantities in terms of photon $\mathrm{GF} \mathcal{D}^{\gamma}$, e.g., for the LDOS

$$
\mathscr{D}_{\gamma}(\mathbf{r} ; E)=-\frac{2 E}{\pi \hbar^{2} c^{2}} \operatorname{tr}\left\{\Im \mathcal{D}^{\gamma, R}(\mathbf{r}, \mathbf{r} ; E)\right\}
$$

the Poynting vector $(\alpha \in\{x, y, z\})$

$$
\begin{aligned}
& \left\langle S_{\alpha}(\mathbf{r})\right\rangle=\int_{0}^{\infty} d E E \sum_{\beta \neq \alpha} \lim _{\mathbf{r}^{\prime} \rightarrow \mathbf{r}}\left[\nabla _ { \mathbf { r } ^ { \prime } } ^ { \beta } \left\{\mathcal{D}_{\beta \alpha}^{\gamma,>}\left(\mathbf{r}, \mathbf{r}^{\prime} ; E\right)\right.\right. \\
& \left.+\mathcal{D}_{\beta \alpha}^{\gamma,<}\left(\mathbf{r}, \mathbf{r}^{\prime} ; E\right)-\mathcal{D}_{\alpha \beta}^{\gamma,>}\left(\mathbf{r}^{\prime}, \mathbf{r} ; E\right)-\mathcal{D}_{\alpha \beta}^{\gamma,<}\left(\mathbf{r}^{\prime}, \mathbf{r} ; E\right)\right\} \\
& -\nabla_{\mathbf{r}^{\prime}}^{\alpha}\left\{\mathcal{D}_{\beta \beta}^{\gamma,>}\left(\mathbf{r}, \mathbf{r}^{\prime} ; E\right)+\mathcal{D}_{\beta \beta}^{\gamma,<}\left(\mathbf{r}, \mathbf{r}^{\prime} ; E\right)\right. \\
& \left.\left.-\mathcal{D}_{\beta \beta}^{\gamma,>}\left(\mathbf{r}^{\prime}, \mathbf{r} ; E\right)-\mathcal{D}_{\beta \beta}^{\gamma,<}\left(\mathbf{r}^{\prime}, \mathbf{r} ; E\right)\right\}\right] /(4 \pi \hbar)
\end{aligned}
$$

and the net radiative rate

$$
\begin{aligned}
& \mathcal{R}_{\mathrm{tot}}^{\mathrm{opt}}=\int \frac{d E}{2 \pi \hbar} \sum_{\mu, \nu} \int d^{3} r \int d^{3} r^{\prime}\left[\mathcal{D}_{\mu \nu}^{\gamma,<}\left(\mathbf{r}, \mathbf{r}^{\prime} ; E\right)\right. \\
& \left.\times \Pi_{\nu \mu}^{\gamma,>}\left(\mathbf{r}^{\prime}, \mathbf{r} ; E\right)-\mathcal{D}_{\mu \nu}^{\gamma,>}\left(\mathbf{r}, \mathbf{r}^{\prime} ; E\right) \Pi_{\nu \mu}^{\gamma,<}\left(\mathbf{r}^{\prime}, \mathbf{r} ; E\right)\right] .
\end{aligned}
$$

Again, the GF in the above expressions are obtained from the steady-state version of Eq. (19): For the dyadic photon GF the integral form of the Dyson equation becomes (summation over repeated indices)

$$
\begin{aligned}
\mathcal{D}_{\mu \nu}^{\gamma, R}\left(\mathbf{r}, \mathbf{r}^{\prime} ; E\right) & =\mathcal{D}_{0, \mu \nu}^{\gamma, R}\left(\mathbf{r}, \mathbf{r}^{\prime} ; E\right) \\
& +\int d^{3} r_{1} \int d^{3} r_{2}\left[\mathcal{D}_{0, \mu \alpha}^{\gamma, R}\left(\mathbf{r}, \mathbf{r}_{1} ; E\right)\right. \\
& \left.\times \Pi_{\alpha \beta}^{\gamma, R}\left(\mathbf{r}_{1}, \mathbf{r}_{2} ; E\right) \mathcal{D}_{\beta \nu}^{\gamma, R}\left(\mathbf{r}_{2}, \mathbf{r}^{\prime} ; E\right)\right]
\end{aligned}
$$

and the kinetic equation for the correlation functions reads

$$
\begin{aligned}
& \mathcal{D}_{\mu \nu}^{\gamma, \lessgtr}\left(\mathbf{r}, \mathbf{r}^{\prime} ; E\right)=\int d^{3} r_{1} \int d^{3} r_{2} \mathcal{D}_{\mu \alpha}^{\gamma, R}\left(\mathbf{r}, \mathbf{r}_{1} ; E\right) \\
& \times\left[\Pi_{0, \alpha \beta}^{\gamma, \lessgtr}\left(\mathbf{r}_{1}, \mathbf{r}_{2} ; E\right)+\Pi_{\alpha \beta}^{\gamma, \lessgtr}\left(\mathbf{r}_{1}, \mathbf{r}_{2} ; E\right)\right] \mathcal{D}_{\beta \nu}^{\gamma, A}\left(\mathbf{r}_{2}, \mathbf{r}^{\prime} ; E\right) .
\end{aligned}
$$

The steady-state form of the photon self-energy energy terms are:

$$
\begin{aligned}
& \Pi_{\alpha \beta}^{\gamma, \lessgtr}\left(\mathbf{r}, \mathbf{r}^{\prime} ; E\right)=i \hbar \mu_{0}\left(\frac{e}{m_{0}}\right)^{2} \lim _{\mathbf{r}^{\prime \prime} \rightarrow \mathbf{r}} \frac{1}{2}\left\{\hat{p}_{\alpha}(\mathbf{r})-\hat{p}_{\alpha}\left(\mathbf{r}^{\prime \prime}\right)\right\} \\
& \times \lim _{\mathbf{r}^{\prime \prime \prime} \rightarrow \mathbf{r}^{\prime}} \hat{p}_{\beta}\left(\mathbf{r}^{\prime \prime \prime}\right) \int \frac{d E^{\prime}}{\pi \hbar} G^{\lessgtr}\left(\mathbf{r}, \mathbf{r}^{\prime} ; E^{\prime}\right) G^{\gtrless}\left(\mathbf{r}^{\prime \prime \prime}, \mathbf{r}^{\prime \prime} ; E^{\prime}-E\right)
\end{aligned}
$$

for the influence of absorption and emission in the material, and

$$
\begin{aligned}
& \Pi_{0, \mu \nu}^{\gamma, \lessgtr}\left(\mathbf{r}, \mathbf{r}^{\prime} ; E\right)=\int d^{3} r_{1} \int d^{3} r_{2} \sum_{\alpha \beta}\left[\left\{\mathcal{D}_{0, \mu \alpha}^{\gamma, R}\right\}^{-1}\left(\mathbf{r}, \mathbf{r}_{1} ; E\right)\right. \\
& \left.\times \mathcal{D}_{0, \alpha \beta}^{\gamma, \lessgtr}\left(\mathbf{r}_{1}, \mathbf{r}_{2} ; E\right)\left\{\mathcal{D}_{0, \beta \nu}^{\gamma, A}\right\}^{-1}\left(\mathbf{r}_{2}, \mathbf{r}^{\prime} ; E\right)\right]
\end{aligned}
$$

for the self-energy components related to the solution of the homogeneous problem, i.e., to incident fluctuations that are independent from the state of the absorber $[59-61]^{+}$.

The formalism as presented here is incomplete insofar as it contains only the self-energies for interactions of charge carriers with photons and phonons. Many more interactions have been studied in the literature on NEGF, such as ionized impurity scattering and alloy-disorder scattering [44], or electron-electron interaction [20, 40, 41, 62, 63]. However, these terms do either not change the qualitative picture of photovoltaic device operation, as in the case of the former two effects, or cannot be handled numerically without severe simplification.

\subsection{Numerical implementation}

4.2.1. General algorithm The central task of any solar cell device simulation based on NEGF is the numerical evaluation of the Dyson and Keldysh equations (38)-(39) and (50)-(51) for the electron and photon Green's functions and of the self-energy terms such as (45), (46) and (52) entering these equations. This general solution procedure is sketched in Fig. 2 for the electronic part. The global parameters that remain constant concern the electronic structure of the constituent materials, including doping profiles, and the device geometry. The external conditions for a specific simulation run are given by the bias voltage, i.e., the separation of the chemical potentials at the contacts which controls the level of carrier injection, and the external illumination spectrum. The computation of the GF then proceeds in two nested self-consistency loops. In the outer loop, the electrostatic potential entering the electronic Hamiltonian is obtained by solving Poisson's equation with the electronic density provided by the GF expression (34). After a suitable choice of an initial potential to set the band profile, the contact selfenergy components can be determined. Since they depend on the electrostatic potential, they have to be updated after each outer iteration step. The second self-consistency loop represents the iterative solution of the equations for Green's functions and self-energies. It should be noted at this point that this iteration is a consequence of the choice of a self-consistent self-energy and alternative conserving schemes exist [64-67]. However, the self-consistent approach not only ensures current conservation after convergence, but in addition provides an insightful way to monitor the evolution of the system towards the steady state [68]. In Fig. 2, only the electronic part is shown,

\footnotetext{
+ Please note that the quantities in (53) are not the standard inverse of the non-interacting photon GF, as integration of their product yields the transverse delta function.
} 


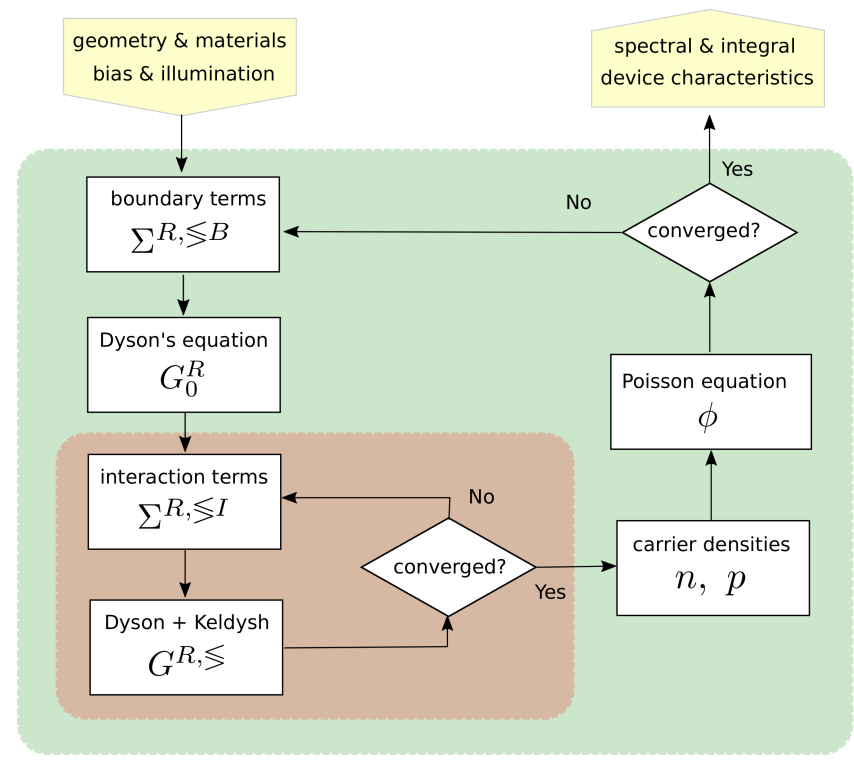

Figure 2. Computational scheme for the evaluation of device characteristics for a given device configuration and operating point determined by external illumination and bias voltage. The general algorithm consists of an outer loop for the self-consistent evaluation of the charge density by coupling to Poisson's equation, and the inner self-consistency iteration of Green's functions and interaction self-energies. Physical quantities such as currents and rates can be obtained in a post-processing step after convergence is reached. Reproduced with permission from Ref. [68].

as with the current-voltage characteristics it provides the key quantities to assess the solar cell device performance. However, if processes such as photon recycling become important, the renormalization of the photon propagator due to occupation of photon modes as a result of spontaneous emission needs to be included in the inner self-consistency iteration via solution of Eqs. (50) and (51) for a photon self-energy (52) that is updated with the actual electronic GF. Once the self-consistency iteration has converged, the physical quantities of interest can all be computed from the GF in a post-processing step using the expressions of the previous section.

4.2.2. Choice of basis In Sec. 4.1, the representation was kept in real space to be as general as possible. However, numerical solution of the NEGF problem requires the application of a discretization scheme for functions and operators, in order to turn the integrodifferential equations into a system that can be solved by optimized linear algebra methods. Conventionally, this is either achieved by application of a real space scheme such as the finite-difference or finite element methods, or by a suitable choice of a finitesize basis such as provided by localized atomic-like orbitals or Wannier functions. The suitability of a given approach strongly depends on the spatial extension and dimensionality of the system, and on the requirements in terms of interactions and necessary phase-space resolution. As a general feature of optoelectronic applications, electronic structure has to be considered on the level of a multiband model, as interband processes are essential for the device operation. Furthermore, the solar spectrum potentially enables optical transitions in a energy range from $0 \mathrm{eV}$ to more than $4 \mathrm{eV}$. For most conventional absorber materials with band gaps around the optimum value predicted by the Shockley-Queisser limit, this means that a large fraction of the Brillouin zone contributes to carrier generation, which favors full-zone models such as tight-binding over k-point expansions such as $\mathbf{k} \cdot \mathbf{p}$ methods. In the presence of complex interfaces or materials with strong compositional or structural disorder, atomistic models are indispensable. Last but not least, microscopic details beyond the envelope function approximation appear in the scattering selfenergies, such as dipole matrix elements or electronphonon coupling constants, which ideally should be evaluated in a consistent fashion.

4.2.3. Numerical challenges The NEGF formalism, while figuring among the most advanced and powerful methods for optoelectronic device simulation, provides its unique insight at the expense of formidable computational cost. The main factors for the immense requirement of computational resources are, on the one hand, the large number and domain of arguments to be resolved - two spatial arguments plus energy - owing to the microscopic nature of the approach, and, on the other hand, the complexity of the self-consistent solution procedure. While the size of the GF is demanding in terms of memory, the coupling in energy, and, in the case of planar structures, transverse momentum which originates in the self-consistency iteration prevents straightforward partition of the problem for solution on large-scale distributed memory supercomputers. In effect, the efficient parallelization of the NEGF approach to the simulation of nano-electronic devices featuring inelastic interactions is under active investigation [69]. In view of the slow convergence of the self-consistency iteration process in the case of strong interactions or the presence of localized states [68], massive parallelization is imperative to enable predictive simulations of realistically sized structures under full spectrum illumination. On the other hand, current implementations of the NEGF formalism for photovoltaic devices limit its applications to structures of mesoscopic spatial extension and with simplified electronic structure models. 
4.2.4. Common pitfalls Due to to the formal and numerical complexity and the computational expense of the approach, a variety of approximations, simplifications and coarse-graining methods are commonly used in implementations of the NEGF formalism. Here, we discuss potential pitfalls arising from three different origins: numerical resolution, parameter ranges, and non-locality. A useful discussion of related issues can also be found in Ref. [70].

Numerical resolution refers to the step size in the spatial, momentum and energy grids. Insufficient refinement in those meshes can lead to incomplete resolution of local features in real, momentum and energy spaces, such as the resonances arising due to localization induced by heterostructure potentials or quantized interactions. Failure to resolve such sharp features can lead to large errors in the physical properties computed based on the spectral quantities, and to problems with the convergence of the selfconsistency iteration. Most importantly, it can result in a violation of current conservation, which is one of the most stringent consistency tests for any NEGF implementation.

Not only the resolution of the grids is important, but also the extent of the meshes. Both energy and momentum meshes need to be chosen large enough as to contain all states and associated transitions of interest. For instance, the momentum range required to describe heavy holes in GaAs within a given energy window around the valence band maximum is much larger than that required for electrons in the same window around the conduction band minimum.

While the above issues are present in any simulation based on (steady-state) NEGF, there is an additional complexity arising in simulations of systems with transitions between (sub-)bands, which has to do with the consideration of non-locality. As soon as objects depend on off-diagonal GF elements, such as in the case of electron-photon interaction selfenergies and interband polarization functions, it is no longer safe to neglect non-locality, i.e., a larger number of off-diagonal elements of the GF need to be evaluated. While in the case of intraband scattering, e.g., with optical phonons, the underestimation of coupling strength due to a diagonal approximation can be mitigated by a simple rescaling of the self-energy, this is not possible in the case of interband scattering, as the spectral shape of the joint density of states is very sensitive to the non-locality range [42].

NEGF implementations should therefore always be tested against established semiclassical approaches for situations where the latter are valid, which in general is the case for bulk-like media at low-field conditions. For the simulation of solar cell devices, it is often useful to compute the absorption coefficient and the absorptance based on the GF and use this information together with the photon flux to asses the correctness of the photocurrent and the radiative dark current obtained by NEGF, based on the relations presented in Sec. 3.1.

\section{Numerical results for selected applications}

\subsection{Ultra-thin absorbers}

While epitaxially grown solar cells made of highquality III-V semiconductor materials reach very high conversion efficiencies, the fabrication process is expensive and energy intensive, and raw materials are not in general earth-abundant. One approach to mitigate these problems consists in a severe reduction of material usage by strongly limiting the absorber thickness and compensating for the reduced absorption length via nanophotonic light trapping that enables coupling to resonant waveguide modes. Indeed, almost complete absorption of the incident light could be shown both theoretically $[71,72]$ and experimentally [73-75] for absorber thicknesses in the far subwavelength regime, which sparked a growing activity in ultra-thin solar cell development [76-79]. While the optics of these devices requires special consideration due to the need for extreme absorption enhancement, the ultra-thin solar cells are in general treated as bulk-like from an electronic point of view. However, for active region extension of far below $100 \mathrm{~nm}$, any variation in the band profile away from flat band conditions represents a strong deviation from the bulk situation. For an absorber thickness of $60 \mathrm{~nm}$ and doped regions of $20 \mathrm{~nm}$, the built-in field sustained by the (fully ionized) doping density of $10^{18} \mathrm{~cm}^{-3}$ ranges from around $50 \mathrm{kV} / \mathrm{cm}$ at open circuit voltage to more than $100 \mathrm{kV} / \mathrm{cm}$ at short circuit conditions. Furthermore, contact regions span a significant fraction of the whole device. The question therefore arises whether these solar cells indeed behave like ordinary bulk devices.

To shed light on the photovoltaic characteristics at mesoscale absorber dimensions, a prototypical ultrathin solar cell was modeled as a GaAs $p-i$ - $n$ device with carrier selective contacts formed by electron and hole blocking layers (EBL/HBL) with band offsets $\Delta E_{\mathrm{C} / \mathrm{V}}$, as schematically depicted in Fig. 3(a) [80]. Since this thin film device is prototypical for a planar architecture with quasi-infinite in-plane dimensions, some space is dedicated in the following to reformulate the NEGF approach in a representation that is suitable for quasi1D slab systems.

In layer structures with homogeneous transverse dimensions, the steady-state equations for the Green's functions can be simplified by using a Fourier transform with respect to transverse coordinates. For 


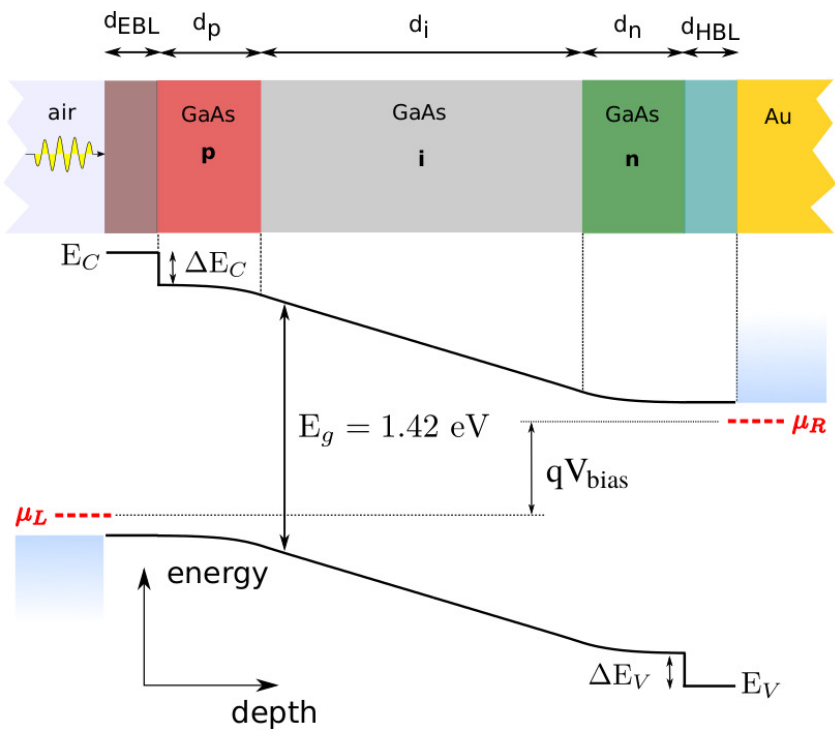

Figure 3. Layer structure and band profile of an ultra-thin GaAs solar cell with electron/hole blocking layers (EBL/HBL) and a gold back reflector. Due to the reduced thickness, the intrinsic absorber region is subjected to very strong built-in electrical fields. The monochromatic illumination is incident from the left assuming perfect in-coupling. The gold mirror is considered explicitly only in the optical simulation. Adapted with permission from Ref. [80].

the charge carriers, the resulting slab representation reads

$$
G\left(\mathbf{r}, \mathbf{r}^{\prime} ; E\right)=\mathcal{A}^{-1} \sum_{\mathbf{k}_{\|}} G\left(\mathbf{k}_{\|}, z, z^{\prime}, E\right) e^{i \mathbf{k}_{\|} \cdot\left(\mathbf{r}_{\|}-\mathbf{r}_{\|}^{\prime}\right)},
$$

where $\mathbf{k}_{\|}$is the transverse quasi-momentum and $\mathcal{A}$ is the slab cross section area. This representation is appropriate here since we consider only the case of quasi-1D planar device architectures. For the photon GF components, the corresponding steady-state slab representation is

$$
\mathcal{D}_{\mu \nu}^{\gamma}\left(\mathbf{r}, \mathbf{r}^{\prime} ; E\right)=\int \frac{d^{2} q_{\|}}{(2 \pi)^{2}} \mathcal{D}_{\mu \nu}^{\gamma}\left(\mathbf{q}_{\|}, z, z^{\prime}, E\right) e^{i \mathbf{q}_{\|} \cdot\left(\mathbf{r}_{\|}-\mathbf{r}_{\|}^{\prime}\right)} .
$$

In the evaluation of physical quantities, integration over transverse momentum needs to be performed, e.g., for the carrier density (upper/lower sign for electrons/holes: $s=c / v$ )

$$
\rho_{s}(z)=\mp i \mathcal{A}^{-1} \sum_{\mathbf{k}_{\|}} \int \frac{d E}{2 \pi} G_{s}^{\lessgtr}\left(\mathbf{k}_{\|}, z, z, E\right),
$$

or the charge carrier current:

$$
\begin{aligned}
j_{s}(z)= & \mp \lim _{z^{\prime} \rightarrow z} \frac{e \hbar}{m_{0}}\left(\partial_{z}-\partial_{z^{\prime}}\right) \\
& \times \mathcal{A}^{-1} \sum_{\mathbf{k}_{\|}} \int \frac{d E}{2 \pi} G_{s}^{\lessgtr}\left(\mathbf{k}_{\|}, z, z^{\prime}, E\right) .
\end{aligned}
$$

In a simple decoupled-band model of the absorber material, the self-energy expression associated with the coherent generation of electrons in the conduction band via transitions mediated by photons of energy $E_{\gamma}$ reads (c/v: conduction/valence band) [42]

$$
\begin{aligned}
\Sigma_{e \gamma, c}^{<\text {(gen })} & \left(\mathbf{k}_{\|}, z, z^{\prime}, E\right)=\left(\frac{e}{m_{0}}\right)^{2} \sum_{\mu} p_{c v}^{\mu}(z) \\
& \times p_{c v}^{\mu *}\left(z^{\prime}\right) G_{v}^{<}\left(\mathbf{k}_{\|}, z, z^{\prime}, E-E_{\gamma}\right) \\
& \times \int \frac{d^{2} q_{\|}}{(2 \pi)^{2}} A_{\mu}\left(\mathbf{q}_{\|}, z, E_{\gamma}\right) A_{\mu}^{*}\left(\mathbf{q}_{\|}, z^{\prime}, E_{\gamma}\right),
\end{aligned}
$$

where $p_{c v}^{\mu}, \mu \in\{x, y, z\}$ are interband momentum matrix elements, and $\mathbf{A}$ is the (modal) vector potential, with $\mathbf{q}_{\|}$denoting the in-plane photon momentum. A similar expression exists for the self-energy of generation of holes in the valence band, with " $<$ " and "c" replaced by ">" and "v", and an energy argument $E+E_{\gamma}$ in the GF. The self-energy encoding radiative recombination of electrons in the conduction band due to the incoherent coupling to field fluctuations acquires the form [42]

$$
\begin{aligned}
& \Sigma_{e \gamma, c}^{>(\mathrm{rec})}\left(\mathbf{k}_{\|}, z, z^{\prime}, E\right)=\left(\frac{e}{m_{0}}\right)^{2} \sum_{\mu, \nu} p_{c v}^{\mu}(z) p_{c v}^{\nu *}\left(z^{\prime}\right) \\
& \times \int d E_{\gamma}\left[G_{v}^{>}\left(\mathbf{k}_{\|}, z, z^{\prime}, E-E_{\gamma}\right)\right. \\
& \left.\times \int \frac{d^{2} q_{\|}}{(2 \pi)^{2}} i \hbar \mu_{0} \mathcal{D}_{\mu \nu}^{\gamma,>}\left(\mathbf{q}_{\|}, z, z^{\prime}, E_{\gamma}\right)\right] .
\end{aligned}
$$

Again, a corresponding in-scattering self-energy for the recombination of holes in the valence band is obtained by the above replacements. If the influence of resonator modes can be neglected, it is common to adopt the approximation of equilibrium free field modes, which amounts to using

$$
\int \frac{d^{2} q_{\|}}{(2 \pi)^{2}} \mathcal{D}_{0, \mu \nu}^{\gamma,>}\left(\mathbf{q}_{\|}, z, z^{\prime}, E_{\gamma}\right)=-\frac{i n_{0} E_{\gamma}}{3 \pi \hbar c_{0}} \delta_{\mu \nu}
$$

in (59). The electron-phonon scattering self-energies are used in the form $(s=c, v)$

$$
\begin{aligned}
\Sigma_{e p, s}^{\lessgtr}\left(\mathbf{k}_{\|}, z, z^{\prime}, E\right)=\frac{1}{V} \sum_{\Lambda, \mathbf{Q}}\left|U_{\lambda, \mathbf{k}_{\|}-\mathbf{Q}_{\|}}\right|^{2} e^{-i Q_{z}\left(z-z^{\prime}\right)} \\
\times\left[N_{\Lambda \mathbf{Q}} G_{s}^{\lessgtr}\left(\mathbf{Q}_{\|}, z, z^{\prime}, E \mp \hbar \Omega_{\Lambda \mathbf{Q}}\right)\right. \\
\left.+\left(N_{\Lambda \mathbf{Q}}+1\right) G_{s}^{\lessgtr}\left(\mathbf{Q}_{\|}, z, z^{\prime}, E \pm \hbar \Omega_{\Lambda \mathbf{Q}}\right)\right],
\end{aligned}
$$

where the propagator of the non-interacting equilibrium phonon mode $(\Lambda, \mathbf{Q})$ for polarization $\Lambda$ and wave vector $\mathbf{Q}$ was introduced in (46), with phonon mode occupation number given by the Bose-Einstein distribution $N_{\Lambda \mathbf{Q}}=\left(e^{\hbar \Omega_{\Lambda \mathbf{Q}} / k_{\mathrm{B}} T}-1\right)^{-1}$. This is a valid approximation in the case where the vibrational properties 
are not drastically modified with respect to the equilibrium bulk properties, as in the planar architectures considered here. In the case of optical phonons, the spectrum can be approximated by a single characteristic frequency $\Omega_{\mathrm{op}}$. For polar materials, the screened Fröhlich coupling is used [81]

$$
\left|U_{\Lambda, \mathbf{Q}}\right|^{2}=\frac{\beta Q^{2}}{\left(Q^{2}+Q_{0}^{2}\right)^{2}}, \quad \beta=\frac{e^{2} \hbar \Omega_{\mathrm{op}}}{2 \varepsilon_{0}}\left(\frac{1}{\epsilon_{\infty}}-\frac{1}{\varepsilon}\right),
$$

where $\varepsilon$ is the static dielectric constant and $\varepsilon_{\infty}$ the high frequency dielectric constant. Static screening is introduced via the inverse screening length $Q_{0}$, for which we take the inverse Debye screening length [82],

$$
Q_{0}=\left(\frac{\rho_{s} e^{2}}{\varepsilon \varepsilon_{0} k_{\mathrm{B}} T}\right)^{1 / 2},
$$

where $\rho_{s}$ is the carrier density. For acoustic phonons, deformation potential scattering is assumed, which amounts to the coupling

$$
\left|U_{\Lambda, \mathbf{Q}}\right|^{2}=\frac{D_{\mathrm{ac}}^{2} \hbar Q}{2 \rho_{M} c_{s}},
$$

where $D_{\text {ac }}$ is the deformation potential constant, $\rho_{M}$ is the semiconductor density and $c_{s}$ is the speed of sound. Furthermore, for low energy (elastic) scattering and high temperatures, the expression for the equilibrium phonon propagator can be simplified using $N_{\Lambda \mathrm{Q}} \approx$ $N_{\Lambda \mathbf{Q}}+1 \approx k_{\mathrm{B}} T /\left(\hbar \Omega_{\Lambda \mathbf{Q}}\right) \approx k_{\mathrm{B}} T /(\hbar c Q)$. Finally, diagonal coupling is assumed in real space.

The components of the photon self-energy describing the renormalization of the photon GF due to interaction with the electronic system in terms of interband transitions become

$$
\begin{aligned}
\Pi_{\mu \nu}^{\gamma, \lessgtr}\left(\mathbf{q}_{\|}, z, z^{\prime}, E_{\gamma}\right)= & \mu_{0}\left(\frac{e}{m_{0}}\right)^{2} p_{c v}^{\mu *}(z) p_{c v}^{\nu}\left(z^{\prime}\right) \\
& \times \mathcal{P}_{c v}^{\lessgtr}\left(\mathbf{q}_{\|}, z, z^{\prime}, E_{\gamma}\right)
\end{aligned}
$$

with the interband polarization function (including a factor of two for the summation over spin)

$$
\begin{aligned}
\mathcal{P}_{c v}^{\lessgtr}\left(\mathbf{q}_{\|}, z, z^{\prime}, E_{\gamma}\right) & =-\frac{i \hbar}{\mathcal{A}} \sum_{\mathbf{k}_{\|}} \int \frac{d E}{\pi \hbar}\left[G_{c}^{\lessgtr}\left(\mathbf{k}_{\|}, z, z^{\prime}, E\right)\right. \\
& \left.\times G_{v}^{\gtrless}\left(\mathbf{k}_{\|}-\mathbf{q}_{\|}, z^{\prime}, z, E-E_{\gamma}\right)\right] .
\end{aligned}
$$

The self-energy components related to incident fluctuations that are independent from the state of the absorber are given by

$$
\begin{aligned}
& \Pi_{0, \mu \nu}^{\gamma, \lessgtr}\left(\mathbf{q}_{\|}, z, z^{\prime}, E\right)=\int d z_{1} \int d z_{2}\left\{\left[\mathcal{D}_{0}^{\gamma, R}\right]_{\mu \alpha}^{-1}\left(\mathbf{q}_{\|}, z, z_{1}, E\right)\right. \\
& \left.\times \mathcal{D}_{0, \alpha \beta}^{\gamma, \lessgtr}\left(\mathbf{q}_{\|}, z_{1}, z_{2}, E\right)\left[\mathcal{D}_{0}^{\gamma, A}\right]_{\beta \nu}^{-1}\left(\mathbf{q}_{\|}, z_{2}, z^{\prime}, E\right)\right\} .
\end{aligned}
$$

In the NEGF picture, the absorption coefficient as derived from the net absorption rate can be expressed in terms of the photon self-energy as follows: [83]

$$
\begin{aligned}
\alpha_{\mu}\left(\mathbf{q}_{\|}, z, E_{\gamma}\right) \approx & \frac{\hbar c_{0}}{2 n_{r}\left(\mathbf{q}_{\|}, z, E_{\gamma}\right) E_{\gamma}} \\
& \times \int d z^{\prime} \operatorname{Re}\left[i \hat{\Pi}_{\mu \mu}\left(\mathbf{q}_{\|}, z^{\prime}, z, E_{\gamma}\right)\right]
\end{aligned}
$$

In order to reproduce the bulk absorption coefficient at flat band conditions, it is essential to consider the non-locality of the electronic states in the derivation of (68), i.e., the off-diagonal matrix elements $\left(z \neq z^{\prime}\right)$ of the charge carrier Green's function in the interband polarization function (66) [42]. In terms of a classical incident radiation field and the photon self-energy, the absorptance for a given polarization of a slab of thickness $d=z_{d}-z_{0}$ acquires the form [42]

$$
\begin{aligned}
a_{\mu}\left(\mathbf{q}_{\|}, z_{d}, E_{\gamma}\right)= & \frac{i}{\hbar \mu_{0}} \Phi_{\mu}^{-1}\left(\mathbf{q}_{\|}, z_{0}, E_{\gamma}\right) \int_{z_{0}}^{z_{d}} d z \\
& \times \int_{z_{0}}^{z_{d}} d z^{\prime}\left[A_{\mu}\left(\mathbf{q}_{\|}, z, E_{\gamma}\right) A_{\mu}^{*}\left(\mathbf{q}_{\|}, z^{\prime}, E_{\gamma}\right)\right. \\
& \left.\times \Pi_{\mu \mu}^{>}\left(\mathbf{q}_{\|}, z^{\prime}, z, E_{\gamma}\right)\right]
\end{aligned}
$$

where $\Phi$ is the modal photon flux (units: $s^{-1}$ ). In terms of the photon GF, the absorptance is given by

$$
\begin{aligned}
a_{\mu \nu}\left(\mathbf{q}_{\|}, E_{\gamma}\right)= & -\int d z \int d z^{\prime}\left[\hat{\mathcal{D}}_{v, \mu \nu}^{\gamma}\left(\mathbf{q}_{\|}, z, z^{\prime}, E_{\gamma}\right)\right. \\
& \left.\times \hat{\Pi}_{\nu \mu}\left(\mathbf{q}_{\|}, z^{\prime}, z, E_{\gamma}\right)\right]
\end{aligned}
$$

where $\hat{\mathcal{D}}_{v}$ is the spectral function of vacuum-induced incident fluctuations [61]. Finally, the local volume emission rate of light derives from the radiative recombination rate in the following modal form [84]:

$$
\begin{aligned}
r^{\mu}\left(\mathbf{q}_{\|}, z, E_{\gamma}\right)= & \sum_{\nu} \int d z^{\prime}\left[(2 \pi \hbar)^{-1} \mathcal{D}_{\mu \nu}^{\gamma,>}\left(\mathbf{q}_{\|}, z, z^{\prime}, E_{\gamma}\right)\right. \\
& \left.\times \Pi_{\nu \mu}^{<}\left(\mathbf{q}_{\|}, z^{\prime}, z, E_{\gamma}\right)\right]
\end{aligned}
$$

For the evaluation of luminescence, the propagation of light inside the cell is required. This information is encoded in the NEGF version of the Poynting vector component $S_{z}(z)=\int d E_{\gamma} \mathcal{S}_{z}\left(z, E_{\gamma}\right)$ for the energy flux normal to the slab surface, which in terms of the photon Green's functions is given by [60]

$$
\begin{aligned}
\mathcal{S}_{z}\left(z, E_{\gamma}\right)= & \frac{E_{\gamma}}{2 \pi \hbar} \int \frac{d^{2} q_{\|}}{(2 \pi)^{2}} s_{z}\left(\mathbf{q}_{\|}, z, E_{\gamma}\right), \\
s_{z}\left(\mathbf{q}_{\|}, z, E_{\gamma}\right)= & -\lim _{z^{\prime} \rightarrow z} \partial_{z^{\prime}} \operatorname{Re} \sum_{\mu=x, y}\left[\mathcal{D}_{\mu \mu}^{\gamma,>}\left(\mathbf{q}_{\|}, z, z^{\prime}, E_{\gamma}\right)\right. \\
& \left.+\mathcal{D}_{\mu \mu}^{\gamma,<}\left(\mathbf{q}_{\|}, z, z^{\prime}, E_{\gamma}\right)\right] .
\end{aligned}
$$


The spectral rate of photon emission normal to the left surface (at $z=z_{0}$ ) into modes coupling to normally incident light is then provided by

$$
\mathfrak{R}_{\mathrm{em}}^{\perp, \text { Poynt }}\left(E_{\gamma}\right)=-s_{z}\left(\mathbf{0}, z_{0}, E_{\gamma}\right) /(2 \pi \hbar)
$$

with the modal Poynting vector computed directly from the photon Green's functions via (73).

NEGF characteristics of ultrathin absorber devices are evaluated for a two-band effective mass Hamiltonian (see Ref. [32] for details on material parameters and discretization) and compared to currentvoltage curves obtained from the semi-classical driftdiffusion-Poisson model under consideration of identical electronic structure for the bulk materials. As can be seen in Fig. 4 for the case of perfectly selective contacts $\left(\Delta E_{\mathrm{C}, \mathrm{V}} \rightarrow \infty\right)$, discrepancies between the predictions of the two pictures are present both in the dark and under monochromatic illumination with photons of energy close to the band gap $\left(E_{\gamma}=1.44 \mathrm{eV}\right)$. The voltage dependence of the photocurrent and the increase in dark saturation current in the NEGF simulation can be traced back to the field dependence of absorption and emission characteristics: Fig. 5(a) shows the sizable deviation of the NEGF absorption coefficient and emitted photon flux evaluated at the center of the intrinsic region from the corresponding quantities based on the flat band bulk model and the generalized Kirchhoff law [85]. The strong variation of the electronic structure with the operating point eventually leads to the breakdown of the photovoltaic reciprocity relation [14] between external photovoltaic quantum

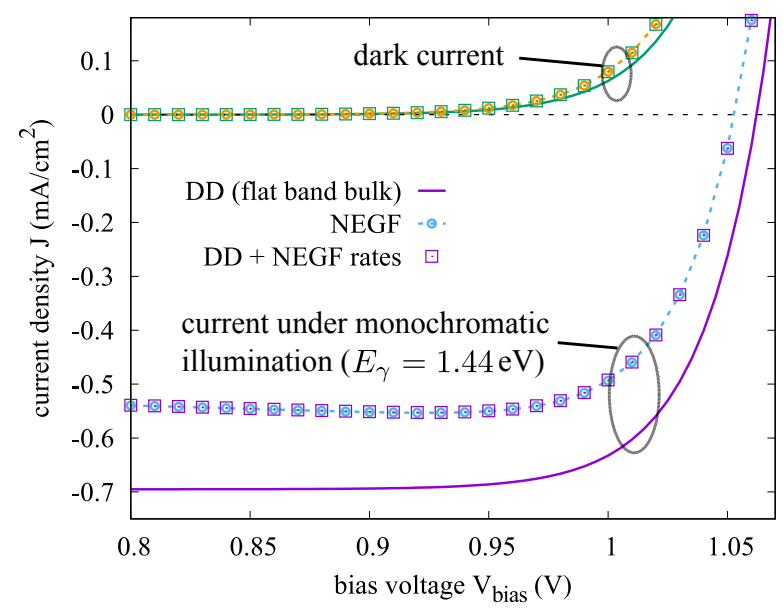

Figure 4. Comparison of NEGF (dashed line with full symbols) and drift-diffusion (full line) characteristics under monochromatic illumination with photon energy close to the band gap $\left(E_{\gamma}=1.44 \mathrm{eV}\right)$ reveals discrepancies in both dark and photocurrents. Use of the rates as derived from NEGF within the drift-diffusion picture of transport reproduces the full NEGF characteristics, which points at band-like transport with unit photocarrier extraction efficiency. Adapted with permission from Ref. [32]. efficiency - or, in the case of the radiative limit, the absorptance - and the photon flux emitted from the surface of the solar cell. Figure 5(b) displays the absorptance of normally incident light (right axis) as well as the photon flux emitted normal to the left surface close to the maximum power voltage. The absorptance $a_{\mathrm{NEGF}}$ computed based on the full NEGF treatment (Eq. (70), [42]) is validated with a transfer-matrix method $\left(a_{\text {TMM }}\right)$. Application of the generalized Kirchhoff law [13] reproduces the emission spectrum, if the bias dependence of the absorptance is considered, while the generalized Kirchhoff spectrum produced based on the absorptance at zero bias is strongly broadened and red-shifted as compared to the NEGF spectrum. Due to the consideration of reabsorption in the NEGF treatment of light propagation inside the cell, the emission computed from the total radiative recombination rate (dash-dotted line) exceeds the emission at the left surface as evaluated from the NEGF-based Poynting vector using Expr. (74) [15].

However, field effects alone cannot explain the entire discrepancy between the semiclassical bulk characteristics and the NEGF result: even if fields are considered in the absorption coefficient via the use of Airy functions [32], the level of dark- and photocurrent generation is different in the two cases. Spatial resolution of generation and recombination reveals a sizable impact of contact regions [80], as displayed in Fig. 6. In the configuration corresponding to perfectly carrier selective contacts $\left(\Delta E_{\mathrm{C}, \mathrm{V}} \rightarrow \infty\right)$, the photocurrent is perfectly rectified, which is shown in the spectral current flow in Fig. 6(a). However, infinite barriers cannot be realized in practice. On the other hand, complete absence of blocking layers causes the appearance of dark and photocurrent leakage through minority carrier contacts (positive current component in the energy range above the top of the band edge at the $p$-contact), which strongly reduces the open circuit voltage, as shown in Fig. 6(b). Introduction of realistic blocking layers with suitable band offsets, such as $\mathrm{Al}_{40} \mathrm{Ga}_{60} \mathrm{As}$ for the EBL and $\mathrm{In}_{49} \mathrm{Ga}_{51} \mathrm{P}$ for the HBL, with both $\Delta E_{\mathrm{C}}$ and $\Delta E_{\mathrm{V}}$ on the order of $350 \mathrm{meV}$, successfully suppresses the leakage current, recovering the ideal $V_{\mathrm{OC}}$ to a large extent, which can also be seen in Fig. 6(b). However, nonzero barriers at majority carrier contacts induce carrier quantization in the absorber and non-bulk-like extraction at the contacts. More dramatically, the finite-height barriers modify the density of states close to the contacts, which results in a dramatic reduction of the short circuit current due to suppression of absorption in these non-classical regions and, in consequence, of the absorptance of the entire device at photon energies close to the band gap as compared to the device with open contacts [Fig. 6(c)]. 

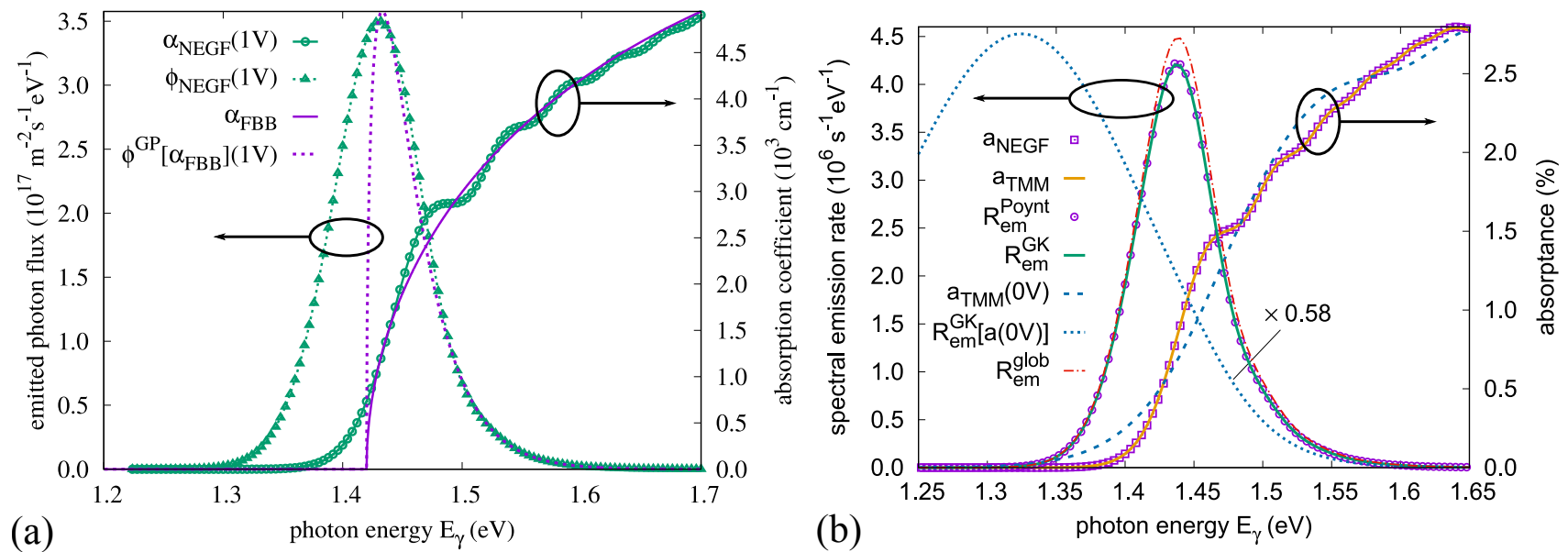

Figure 5. (a) The effects of the strong built-in fields modify both the local values of the absorption coefficient and the emitted photon flux with respect to the corresponding flat band bulk (FBB) properties, where the emission $\phi^{\mathrm{GP}}$ is evaluated using the generalized Planck law. Typical field effects include the appearance of sub-gap tails and oscillations at higher energies in the absorption coefficient, which results in a pronounced broadening and red-shift of the emission spectra. Adapted with permission from Ref. [85]. (b) The field dependence of local properties propagates to a bias dependence of the global absorptance and normal emission at the cell surface. The relation of the two quantities obeys the generalized Kirchhoff law only if the bias dependence is considered. The NEGF absorptance $a_{\text {NEGF }}$ derived in terms of the photon GF agrees with the absorptance $a_{\mathrm{TMM}}$ computed using the transfer-matrix method. Since reabsorption of internally emitted light is included in the NEGF treatment of light propagation, the normally emitted photon flux is lower than the total integrated rate of emission towards the left surface (dash-dotted line). Adapted with permission from Ref. [15].

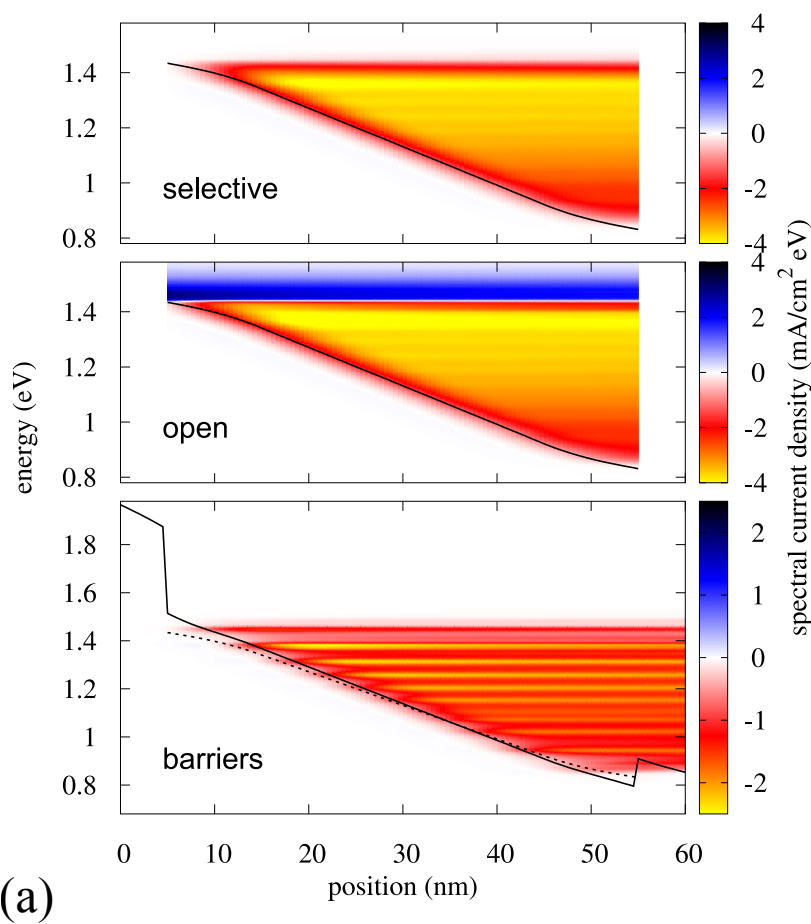

(b)

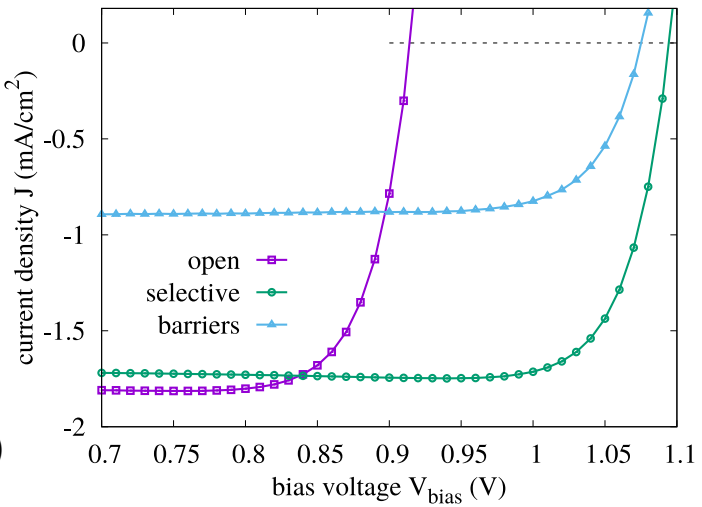

(a)

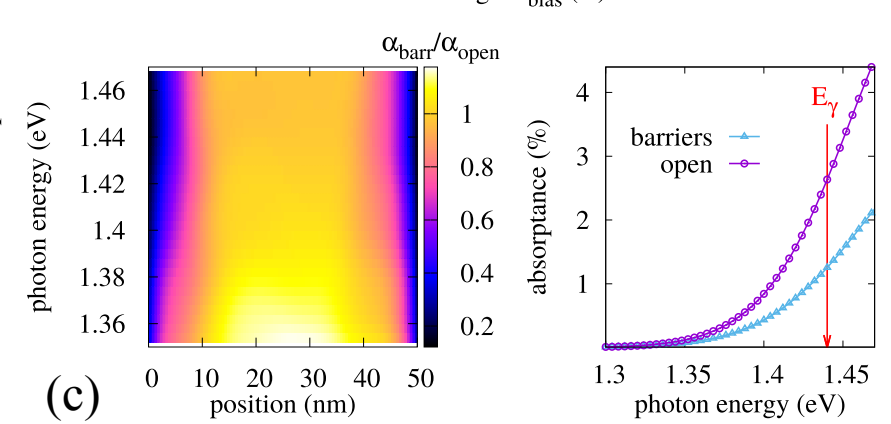

Figure 6. (color online) Impact of contact configuration on the electron component of dark- and photocurrents in ultrathin-absorber solar cells at applied forward bias voltage: (a) For perfectly selective contacts $\left(\Delta E_{\mathrm{C}, \mathrm{V}} \rightarrow \infty\right)$, photocurrent is completely rectified, while leakage currents appear for open minority contacts $\Delta E_{\mathrm{C}, \mathrm{V}}=0$ due to extraction of injected carriers, which results in a severe $V_{\mathrm{OC}}$ loss. (b) While blocking layers successfully prevent leakage of minority carriers, they also degrade the short circuit current. (c) The loss in $J_{\mathrm{SC}}$ can be attributed to the appearance of non-classical interface regions with suppressed absorptance in the vicinity of blocking layers. Reproduced with permission from Ref. [80]. 


\subsection{Quantum well solar cells}

Quantum wells have been investigated for photovoltaic applications mainly in III-V semiconductor material systems and for epitaxial growth. There are two main types of QW solar cells: multi-quantum well (MQW) devices and quantum well superlattice (QWSL) solar cells, which will both be considered in the following. As a special application of quantum well structures in photovoltaic devices, double quantum well tunnel junction (DQW TJ) architectures for multi-junction solar cells are discussed.

\subsubsection{Multi-quantum well solar cells Multi-quantum} well solar cells count among the earliest, prototypical and most successful nanostructure-based solar cell devices. The developments started in the early days of epitaxial growth of optoelectronic devices based on III-V semiconductor heterostructures from alloys of GaAs with $\mathrm{Al}$, In and P [87-92], and have since spread to many new material systems such as, dilute nitrides [93, 94] and nitride alloys [95-100], or antimonides [101-103] and even Si and Ge alloys [104]. While initially MQW solar cells have been claimed to hold the promise of fundamentally higher conversion efficiencies as compared to single junction bulk devices [9], thermodynamical arguments soon showed that that this is not the case [12]. However, MQW absorbers turned out to be of great interest for multi-junction solar cells, where they provide absorber components with largely tunable band gaps for ideal performance optimization [105-111]. The main difference of MQW solar cells to bulk devices is the presence of largely localized states in the absorber region. These states contribute to the generation of electron-hole pairs by extending the absorption range of the bulk host material to longer wavelengths, but do not participate in the transport of free carriers to the contacts: for this purpose, photogenerated carriers first need to be transferred to extended states in the continuum. On the other side, the localized states lead to an increase in recombination - and, in consequence, dark current - due to larger overlap of electron and hole wave functions and local carrier accumulation. Hence, carrier escape and capture processes mediating between QW and continuum states are among the main aspects of MQW solar cell operation to be investigated. Indeed, these processes have been the subject of numerous experiments and modelling studies [112-115]. The common understanding of the relevant regime at room temperature is predominance of thermionic emission [116] over tunneling escape [117], at least up to moderate built-in electric fields $[112,118,119]$. While the conventional models supporting this picture are based on consideration of individual scattering processes between discrete states of idealized QW potentials and a bulk-like continuum with predefined occupation, the real density of states and occupation function reflect the conditions of operation in terms of bias voltage and the corresponding potential profile, and the actual illumination spectrum. The NEGF approach, in contrast, captures the general picture and can therefore be used to validate (or falsify) the more approximate treatments.

For the operation of MQW, consideration of an isolated single QW (SQW) provides most of the relevant information, up to the effects on the extraction process of recapture in subsequent QW. In the following, the role of the QW depth and of the built-in field in carrier escape and capture is investigated for the situation where the interactions are restricted to electron-photon and electron-phonon scattering, i.e., carrier-carrier scattering is neglected, which is reasonable at low excitation intensity, but might be no longer justifiable in systems with high optical concentration.

Carrier photogeneration in SQW is assessed by consideration of the excess carrier density $\delta n=n-n_{0}$, where $n_{0}$ is the carrier density in the dark, for different energy of the monochromatic illumination. As shown in Fig. 7(a) for the case of a $5 \mathrm{~nm}$ wide AlGaAs-GaAs SQW with band offsets $\Delta E_{\mathrm{C}}=$ $200 \mathrm{meV}$ and $\Delta E_{\mathrm{V}}=150 \mathrm{meV}$ (see Ref. [86] for a complete list of parameters), excitation at the absorption edge of the $\mathrm{QW}\left(E_{\gamma}=1.45 \mathrm{eV}\right)$ leads to a spectral density with pronounced phonon satellites, while excitation at higher energies generates carriers also above the ground state, which results in a broadened spectral excess carrier density. The associated spectral current flow displayed in Fig. 7(b) reflects the photogenerated carrier density and reveals a transition in the photocarrier escape mechanism from phonon-assisted tunneling to direct escape $[86,120]$. For further assessment of the photocarrier escape in SQW, the carrier extraction efficiency is evaluated as the ratio $\eta_{\text {ext }}=J_{\mathrm{sc}} / J_{\mathrm{abs}}$ of short-circuit current $\left(J_{\mathrm{sc}}\right)$ and generation current $\left(J_{\text {abs }}\right)$ at low carrier lifetime in $5 \mathrm{~nm}$ wide GaAs-InGaAs SQW for different values of the built-in field, the band offsets $\Delta E_{\mathrm{C}, \mathrm{V}}$ and photon energy $E_{\gamma}$. The result displayed in Fig. 8 illustrates the beneficial impact of a built-in field on the carrier escape. The effect is more pronounced at large band offsets corresponding to strong confinement, for which thermal escape is ineffective, and persists even at higher excitation energies, which points at carrier relaxation competing with extraction. For shallow QW, on the other hand, fast extraction leads to insensitivity to the field condition, and to absence of relaxation of carrier generated at high energies.

Photovoltaic device characteristics can be ob- 
(a)
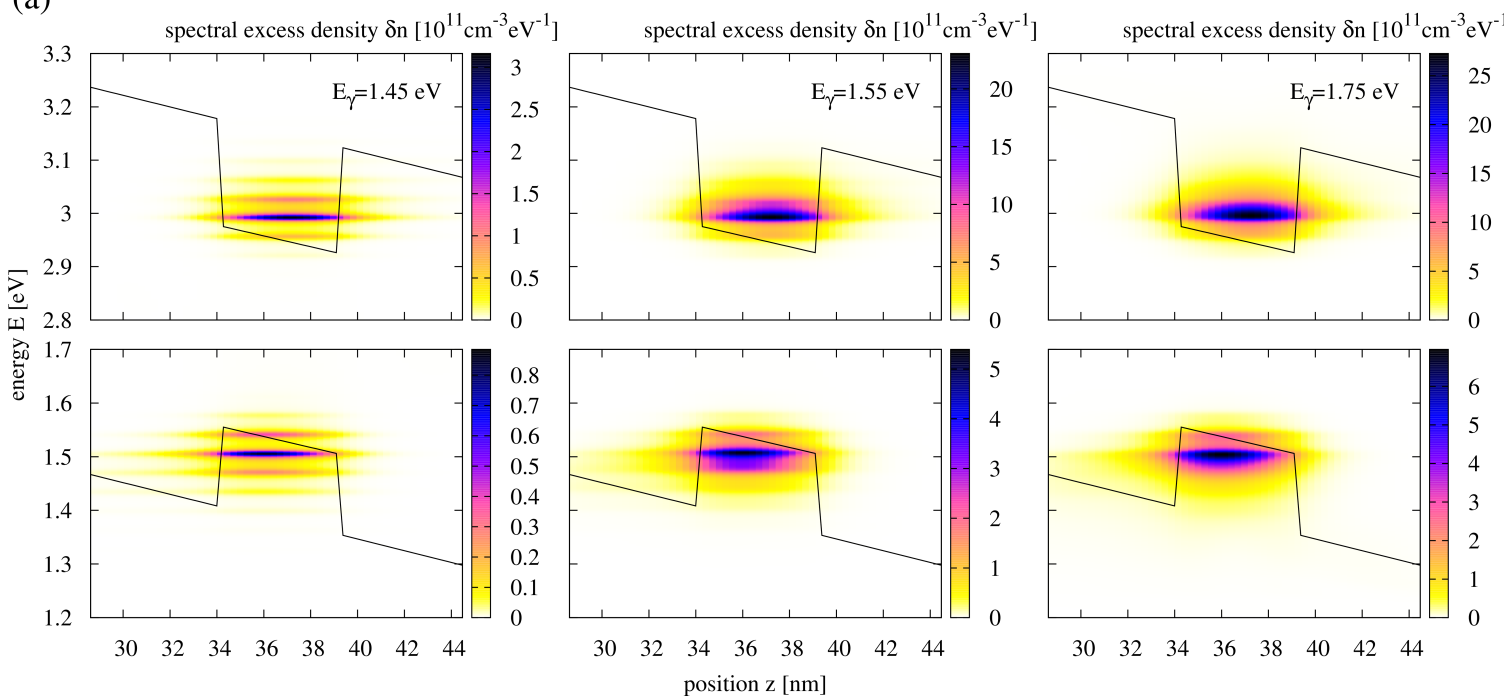

(b)
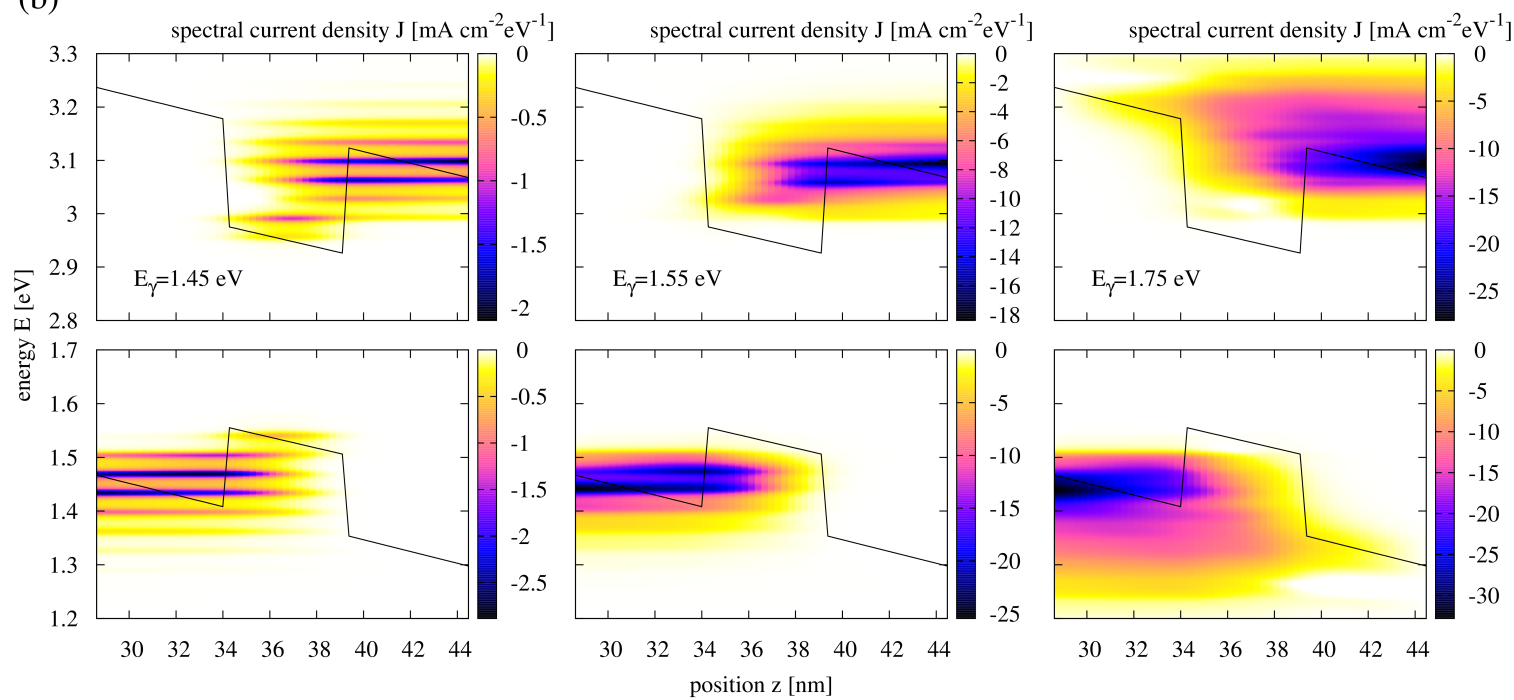

Figure 7. (color online) (a) Photogenerated excess carrier density in a $5 \mathrm{~nm}$ wide AlGaAs-GaAs SQW under monochromatic illumination with different photon energies. At $E_{\gamma}=1.45 \mathrm{eV}$, carriers are generated on the lowest QW states, and the structure in the spectral density is due to phonon satellites. At higher photon energies, carriers are generated at different energies in the QW and subsequently relax to a broadened spectral density at steady state. (b) Corresponding spectral current flow, exhibiting the transition in the escape mechanism from phonon-assisted tunneling at low excitation energy to direct tunneling escape at larger photon energies.

tained by embedding the SQW in the intrinsic region of a $p-i-n$ diode as the one displayed in Fig. 3. In Fig. 9, the current-voltage characteristics are shown for a 10-nm InGaAs QW embedded in a GaAs diode, with band offsets of $\Delta E_{\mathrm{C}}=150 \mathrm{meV}$ and $\Delta E_{\mathrm{V}}=100$ $\mathrm{meV}$, respectively. The illumination is monochromatic with $E_{\gamma}=1.3 \mathrm{eV}$ and at an intensity of $0.1 \mathrm{~kW} / \mathrm{m}^{2}$. The characteristics are evaluated for different theoretical descriptions: the global detailed balance picture (DB) with absorptance from Lambert-Beer law (LB) or the transfer-matrix method (TMM) for the standard textbook square well absorption coefficient $\left(\alpha_{\mathrm{SW}}\right)$; the semiclassical drift-diffusion-Poisson model (DD) coupled to TMM; and the full NEGF-Poisson model. The LB and TMM pictures provide slightly different absorptance levels, and the DD model exhibits an increased dark saturation current due to the emission into the full solid angle as compared to the loss cone in the case of the global models, and the absence of photon recycling. Remarkably, consideration of the absorption coefficient $\alpha_{\mathrm{NEGF}}$ as provided by the NEGF formalism results in semiclassical characteristics that match closely those of the full NEGF model, similar to the situation encountered for the ultra-thin absorber 


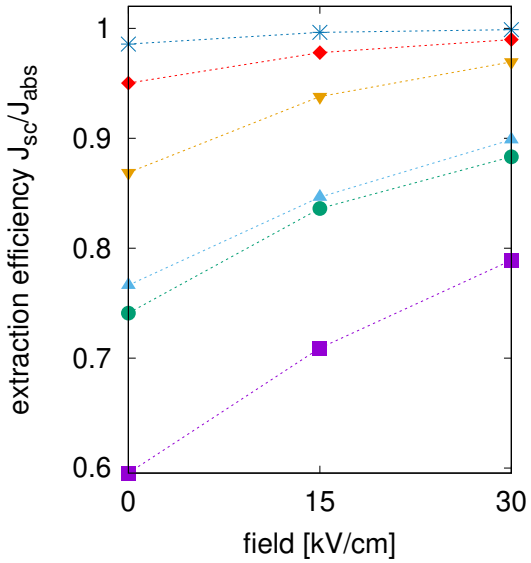

Figure 8. Photocarrier extraction efficiency at low carrier lifetime in a GaAs-InGaAs SQW at short circuit condition and for different values of the built-in field, of the band offsets for electrons and holes, and of the photon energy. The beneficial impact of larger fields is most pronounced at strong confinement, where thermal escape is ineffective even at larger photon energies, in contrast to the situation at low confinement, where thermal escape is able to compete with carrier relaxation to the ground state.

device. This justifies a posteriori the assumption of unit escape probability of carriers generated in quantum well states that is used in the semiclassical model and which is based on experimental observations for device operation at room temperature and moderate well depth [112]. As can be inferred from Fig. 8, this unit extraction efficiency is a consequence of the shallow confinement, strong field and large photon energy in combination with long radiative lifetime, such that the escape is considerably faster than the recombination. However, the presence of ultra-fast non-radiative recombination channels will result in incomplete carrier extraction from deep wells and at large forward bias corresponding to low fields. On the other hand, if the confinement is neglected, as in the semiclassical approach used here, carriers are generated in extended states only and drift or diffuse away quickly, irrespective of the actual potential profile.

In principle, carriers photogenerated in the isolated QWs of a MQW solar cell device can be recaptured by subsequent QWs which they pass on their way to the contacts. In order to asses the efficiency of this process, photocarrier extraction at low carrier lifetime and short-circuit conditions was investigated in GaAs-InGaAs QW solar cell devices consisting of either three isolated QW or the SQW components of this structure, i.e., left, central and right SQW devices. For $\Delta E_{\mathrm{C}}=150 \mathrm{meV}$ and $\Delta E_{\mathrm{V}}=100 \mathrm{meV}$ and $E_{\gamma}=1.25$ $\mathrm{eV}$, the total generation of the three SQW differs very little from the generation in the MQW device, while the

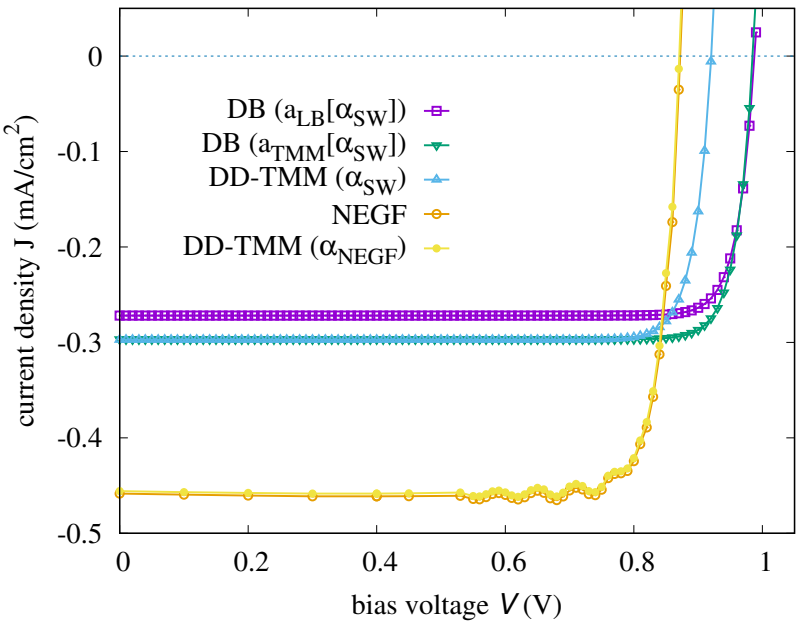

Figure 9. Current-voltage characteristics of a single GaAs-InGaAs quantum well photodiode for monochromatic illumination with $E_{\gamma}=1.3 \mathrm{eV}$ and at an intensity of 0.1 $\mathrm{kW} / \mathrm{m}^{2}$, as provided by: the detailed balance picture (DB) with absorptance from Lambert-Beer law (LB) or the transfermatrix method (TMM) for square well absorption coefficient $\left(\alpha_{S W}\right)$; the semiclassical drift-diffusion-Poisson model (DD) coupled to TMM; and the full NEGF-Poisson model. While the LB and TMM laws provide slightly different absorptance levels, consideration of the absorption coefficient as provided by the NEGF formalism results in semiclassical characteristics that match closely those of the full NEGF model. Reproduced with permission from Ref. [121].

emission is $20 \%$ larger in the MQW case, confirming the detrimental impact of carrier capture.

Since we will consider strongly coupled QW systems below, the transition between isolated and coupled well structures shall be examined here. Figure 10 shows the spectral current flow in GaAs-InGaAs MQW structures $\left(\Delta E_{\mathrm{C}}=150 \mathrm{meV}, \Delta E_{\mathrm{V}}=100 \mathrm{meV}\right.$, $\left.E_{\gamma}=1.2 \mathrm{eV}\right)$ with barrier thickness decreasing from $L_{\mathrm{B}}=15 \mathrm{~nm}$ to $L_{\mathrm{B}}=5 \mathrm{~nm}$. Coupling of QW evidenced by a finite tunnel current components between adjacent QW sets in at around $L_{\mathrm{B}}=12.5 \mathrm{~nm}$. At $L_{\mathrm{B}}=15 \mathrm{~nm}$, carrier extraction is based purely on phonon-assisted thermal escape and hot carrier flow in the quasi-continuum above QW. With shrinking barrier thickness, the tunneling current component increases, while the hot carrier component is reduced and restricted to the extraction process outside the QW region.

\subsubsection{Quantum well superlattice solar cells It was} shown in the last section that for strong confinement in deep QW, thermal escape is no longer efficient. In that situation, carrier extraction via tunneling between QW can be advantageous. In the case of a regular (i.e., periodic) sequence of $\mathrm{QW}$ and barriers, 


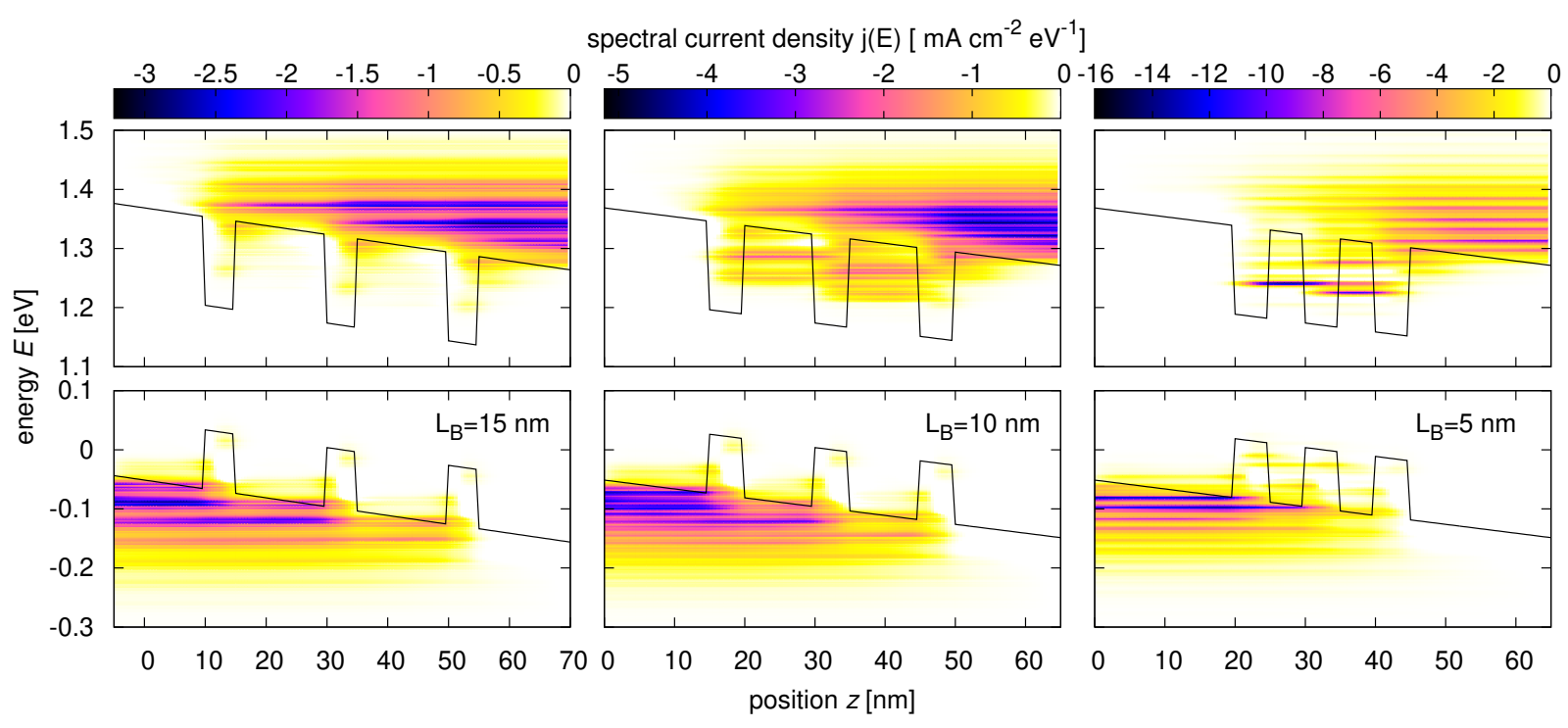

Figure 10. (color online) Evolution of the spectral current flow at photon energy $E_{\gamma}=1.2 \mathrm{eV}$ (generation of confined carriers) in GaAs-InGaAs MQW solar cells with increasing coupling of the QW due to shrinking barrier thickness. At $15 \mathrm{~nm}$ barrier thickness $\left(L_{\mathrm{B}}\right)$, the QW are completely decoupled, and photocurrent flows in the quasi-continuum, following phonon-assisted carrier escape. At $L_{\mathrm{B}}=10 \mathrm{~nm}$, weak coupling of the QW induces a tunneling current component. At $L_{\mathrm{B}}=5 \mathrm{~nm}$, transport in the QW region is dominated by tunneling and the hot carrier flow is strongly reduced except for the extraction at the contacts.

the structure is termed a QW superlattice (QWSL), and transport is assumed to proceed via extended miniband states. However, in realistic systems, the number of periods is finite, and the miniband formation is incomplete. This is displayed in Fig. 11 for the case of a $\mathrm{In}_{0.52} \mathrm{Al}_{0.35} \mathrm{Ga}_{0.15} \mathrm{As}-\mathrm{In}_{0.53} \mathrm{Ga}_{0.47} \mathrm{As}$ system (parameters as in Ref. [84]) with $2.5 \mathrm{~nm}$ well width and

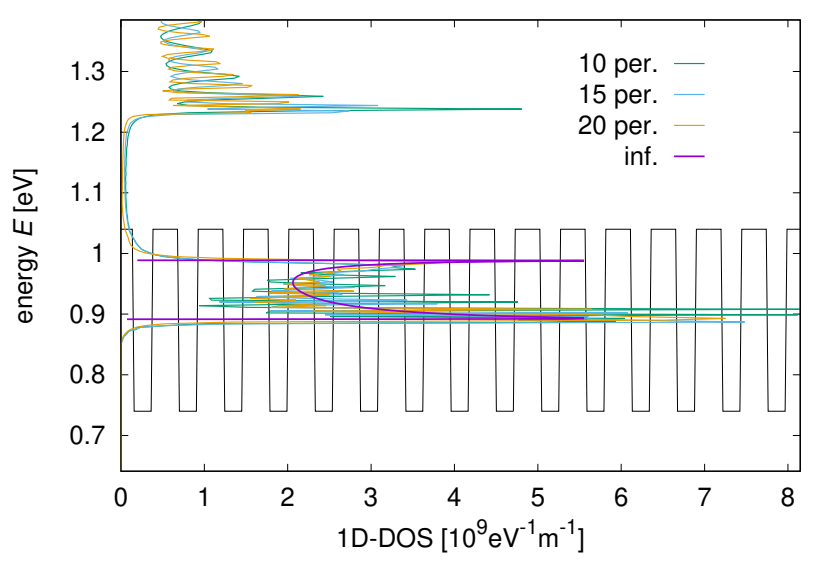

Figure 11. Evolution of the $1 \mathrm{D}$ density of states $\left(\right.$ at $\left.\mathbf{k}_{\|}=0\right)$ for the InAlGaAs-InGaAs SL with QW and barrier thicknesses of $2.5 \mathrm{~nm}$ and $3.5 \mathrm{~nm}$, respectively, with increasing number of periods. For comparison, the result for the perfectly periodic structure is shown as well (inf.). While the bandwidth converges quickly, the structure in the DOS that is related to the finite number of QW remains up to large number of periods.
$3.5 \mathrm{~nm}$ barrier thickness and finite as well as infinite number of periods. For the finite structures, the 1DDOS at $\mathbf{k}_{\|}=0$ is evaluated from the NEGF, assuming contacts made of the barrier material, and exhibits a spectral structure that reflects the number of periods considered, and which converges to the DOS for the infinite - i.e., perfectly periodic - structure, which is computed via solution of the effective mass Schrödinger equation with periodic boundary conditions.

In realistic devices, the potential is not flat at the maximum power point, and the effects of finite built-in fields need to be considered when evaluating the optoelectronic properties. Figures 12 (a) and (b) show the transition from flat band to finite builtin field for the case of the InAlGaAs-InGaAs QWSL with periods of $1.5 \mathrm{~nm}$ thick barriers separating 2.5 $\mathrm{nm}$ wide QW. The local density of states (LDOS) reveals the preservation of the effective band gap increase as compared to the QW material. In this situation of strong coupling of adjacent periods, carrier extraction proceeds quasi-ballistically even at finite field, as can be seen in Fig. 12 (c). However, with increasing barrier thickness, the extraction mechanism changes to sequential tunneling with carrier relaxation in between periods, which makes contact to the regime of transport found when approaching the QWSL from the MQW situation. For the thin barrier QWSL structures, the quasi-ballistic extraction is very fast, which results in unit extraction efficiency and leads to current-voltage characteristics that are the exact superposition of bias-dependent photocurrent 

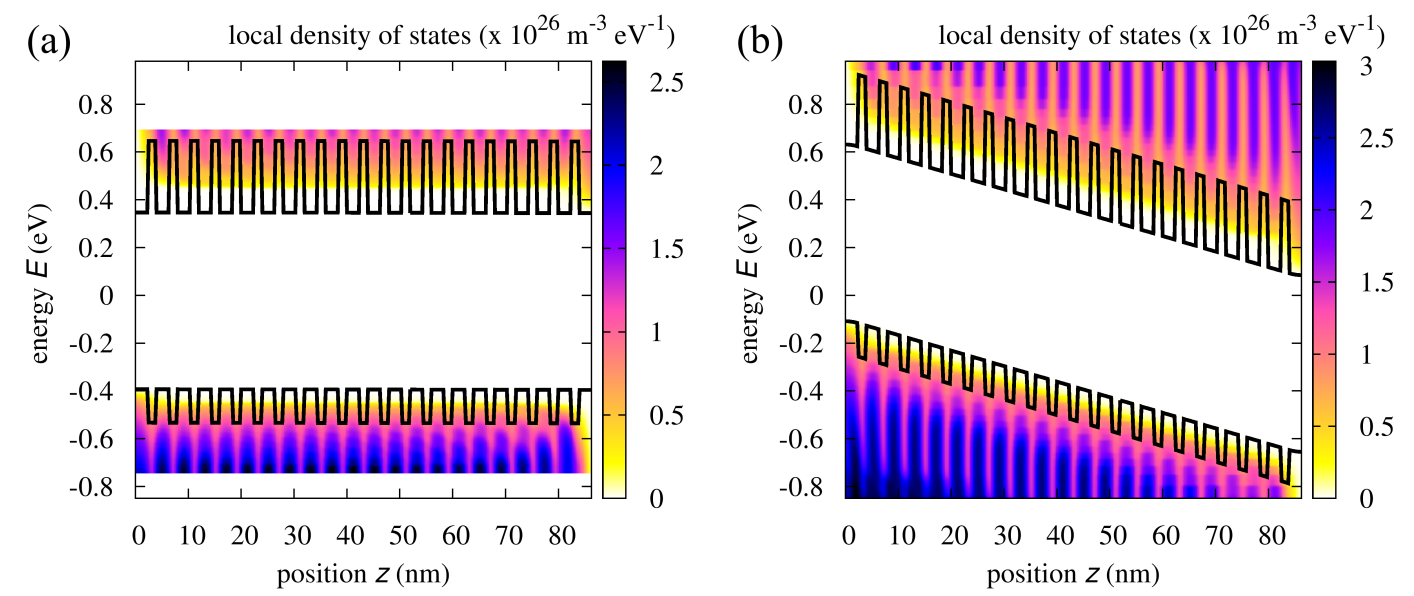

(c)

spectral current density j(E) [ $\left.\mathrm{mA} \mathrm{cm}^{-2} \mathrm{eV}^{-1}\right]$

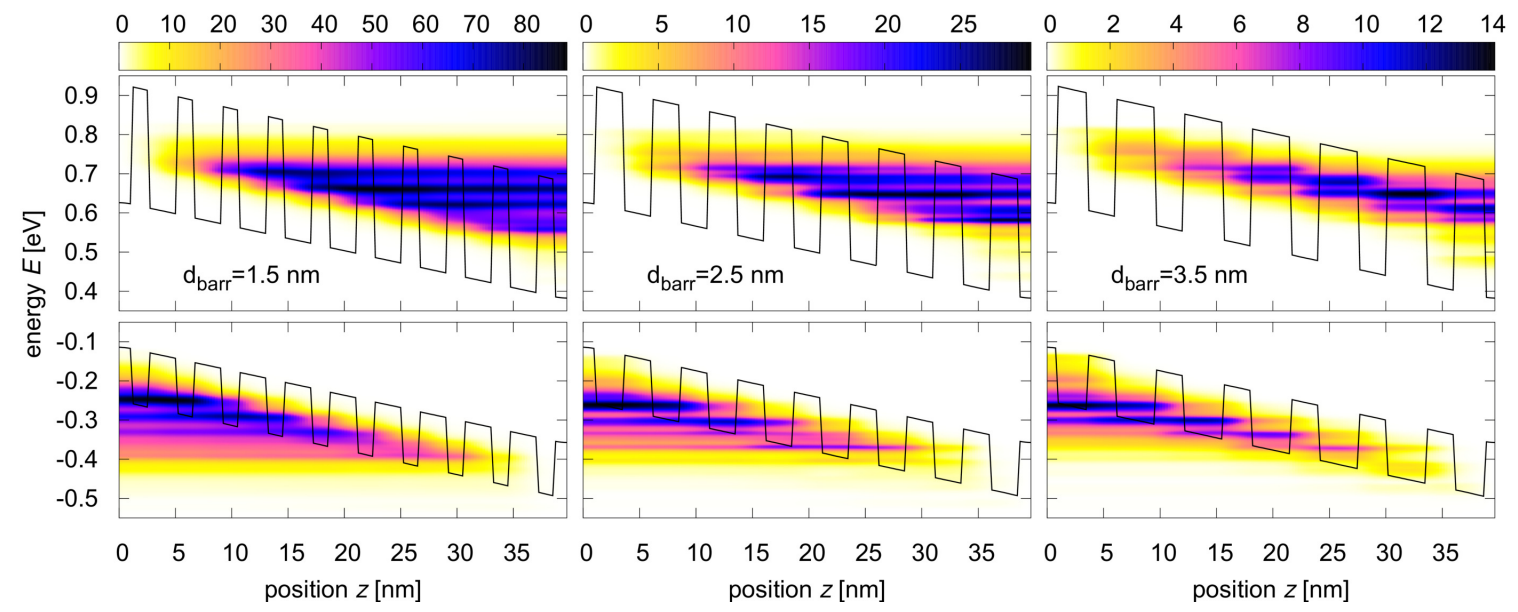

Figure 12. (color online) (a) Local density of states in a 20-period InAlGaAs-InGaAs QWSL with $1.5 \mathrm{~nm} / 3.5 \mathrm{~nm}$ barrier/well thickness, exhibiting the targeted miniband formation at increased effective band gap. (b) In this strong coupling regime, both band gap widening and wave function delocalization are preserved under large built-in fields. (c) Spectral photocurrent flow for the configuration in (b) as well as for larger values of the barrier thickness, revealing a transition of the transport regime from quasiballistic extraction to sequential tunneling with carrier relaxation between periods. Reproduced with permission from Ref. [84].

determined from the absorptance $\left(J_{\text {abs }}\right)$ and the radiative dark current $\left(J_{\text {dark }}\right)$, as shown in Fig. 13. However, as in the case of the ultra-thin absorber device, the voltage-dependance of the photocurrent is significant, and the conventional approach of using the short circuit current in the superposition is not applicable [84].

\subsubsection{Quantum well tunnel junctions Series-connected} multi-junction solar cell architectures require the matching of currents across the entire cell stack. In practice, the connection is achieved via interband tunnel-recombination junctions between subcells, which mediate between electron and hole flows in different subcells. In many cases, multi-junction devices are designed for operation under concentration, which results in large currents to be passed through the junction. The interband tunnel current itself depends criti- cally on the thickness of the junction - i.e., the field sustained by the large gradient in doping - and the band gap of the material: the larger the field and the smaller the gap, the larger the tunnel current that can be sustained. However, both the presence of low band gap material and of large doping concentration required for the high fields induce parasitic absorption losses. In order to reduce such losses in the case of the InP-based multi-junction solar cells, the introduction of a double quantum well (DQW) tunnel junction was proposed, which features a region of lower band gap material, but with lower absorption as compared to bulk [122]. In order to study the nature and role of the QW states in the TJ region, both of the TJ structures investigated experimentally in Ref. [122] and displayed in Fig. 14(a) were simulated using NEGF [33, 123]: an InAlGaAsInGaAs bulk heterojunction with and without InGaAs DQW inserted in the junction region. 


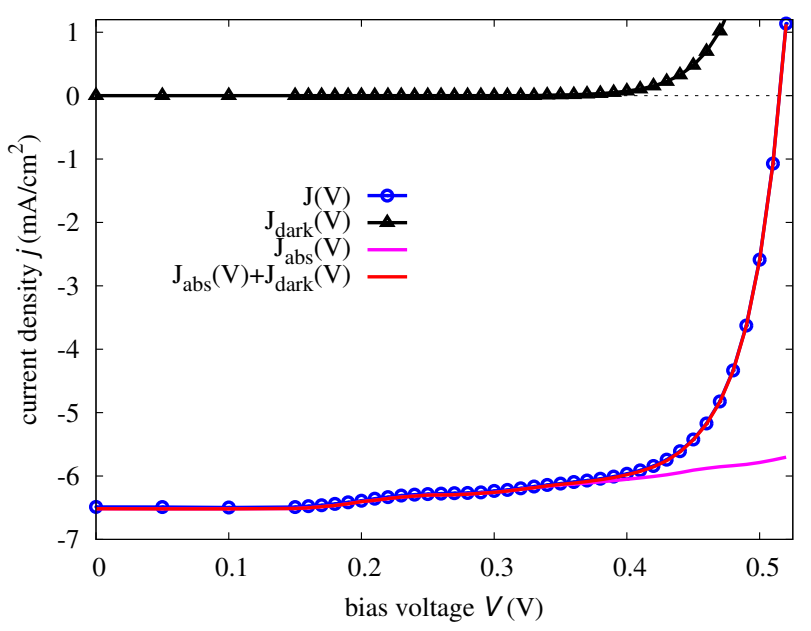

Figure 13. Current-voltage characteristics of the 20period InAlGaAs-InGaAs superlattice in the dark and under monochromatic illumination with photons of energy $E_{\gamma}=0.95$ $\mathrm{eV}$ and at an intensity of $I_{\gamma}=0.1 \mathrm{~kW} / \mathrm{cm}^{2}$. Due to the high degree of wave function delocalization, the carrier escape probability is unity, i.e., the light JV-curve is the exact superposition of the photocurrent from the absorptance and of the radiative dark current. However, it is essential to consider the bias dependence of the photocurrent, as significant deviations from the value at short circuit are observed. Reproduced with permission from Ref. [84].

The main requirement on a model for interband tunneling is a consistent picture of the complex band structure that connects valence and conduction bands. For this reason, a two-band $s p_{z}$ tight-binding model is used for the description of the electronic structure of the bulk constituent materials [25]. In conjunction with the NEGF-Poisson framework applied to the two heterojunctions, it provides a featureful image of the local density of states in the junction region [Fig. 14(b)]. For junctions where direct tunneling provides the main contribution to the interband current, the semiclassical Wentzel-KramersBrillouin (WKB) formalism can reliably predict the current-voltage characteristics, whereas consideration of inelastic electron-phonon interaction - and, hence, a complete NEGF simulation - is required for a valid description of tunneling between different points of the Brillouin zone in indirect gap materials [124], or in the situation of incomplete energetic overlap of bands in left and right reservoirs as in the present case [33]. Indeed, as the band diagrams and LDOS maps for zero bias displayed in Fig. 14(b) show, no direct tunneling is possible already at small forward bias voltage. On the other hand, the phonon-mediated tunneling picture resulting from the NEGF simulation agrees well with the experimental characteristics, as demonstrated in Fig. 14(c), especially regarding the peak voltage and the relative magnitude of currents in bulk and DQW tunnel-heterojunctions. Deviations at large forward bias are due to the onset of trap-assisted tunneling, which presently is not included in the model.

More importantly, the NEGF approach sheds light on the roles of the different localized states in the junction region and of inelastic electron-phonon scattering. For instance, resonance features in the current-voltage characteristics can be attributed to the energetic alignment of the different QW states [Fig. 15(a), left side], and the corresponding spectral current flow [Fig. 15(a), right side] exhibits the signature of the discrete energy of the optical phonons that are absorbed or emitted in the inelastic scattering processes. The absorption characteristics of the heterojunctions are assessed based on the same electronic structure as the tunneling transport. Figure 15(b) shows the local absorption coefficient in the junction region as computed from NEGF for the DQWheterojunction close to zero bias voltage. While the absorption in the reservoir region reflects the parabolic two-band bulk characteristics, the absorption in the junction region exhibits pronounced subband tails due to the strong built-in electric field. This strong deviation from both flat band bulk and square well DQW absorption is evidenced in Fig. 15(c) which displays the absorptance for all three cases, and should be taken into consideration when assessing the absorption losses in tunnel junctions [123].

\subsection{Quantum dot solar cells}

QD offer a large degree of tunability in their optoelectronic properties via adjustment of configurational parameters such as size, shape and composition, and are therefore attractive candidates for the implementation of third-generation photovoltaic concepts. The many applications of QD in photovoltaic devices fall in two main categories: those where the QD have purely optical functionality, e.g., as luminescent species for spectral conversion, and those where the QD states participate in the actual photogeneration process. Representatives of both applications have been studied using NEGF and will be discussed in the following.

5.3.1. Quantum dot fluorescence Uniform ensembles of size-controlled QD are widely considered as luminescent species in luminescent solar concentrator architectures, where they are used to convert broadband to narrow-band illumination tuned to the absorption edge of a specific solar cell material [126]. The key information thereby are the absorption and emission spectra of the QD, the latter in dependence of the optical excitation. In such an application, the functionality of $\mathrm{QD}$ is purely optical, there is no electronic coupling among the QD and no photocarrier 

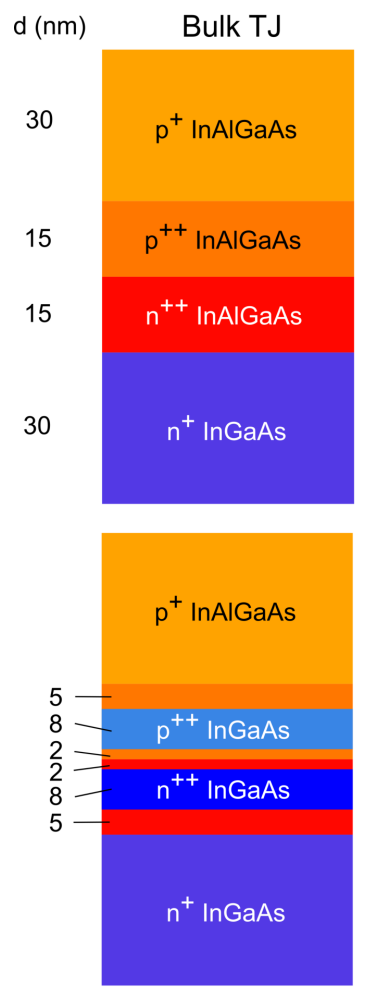

(a)
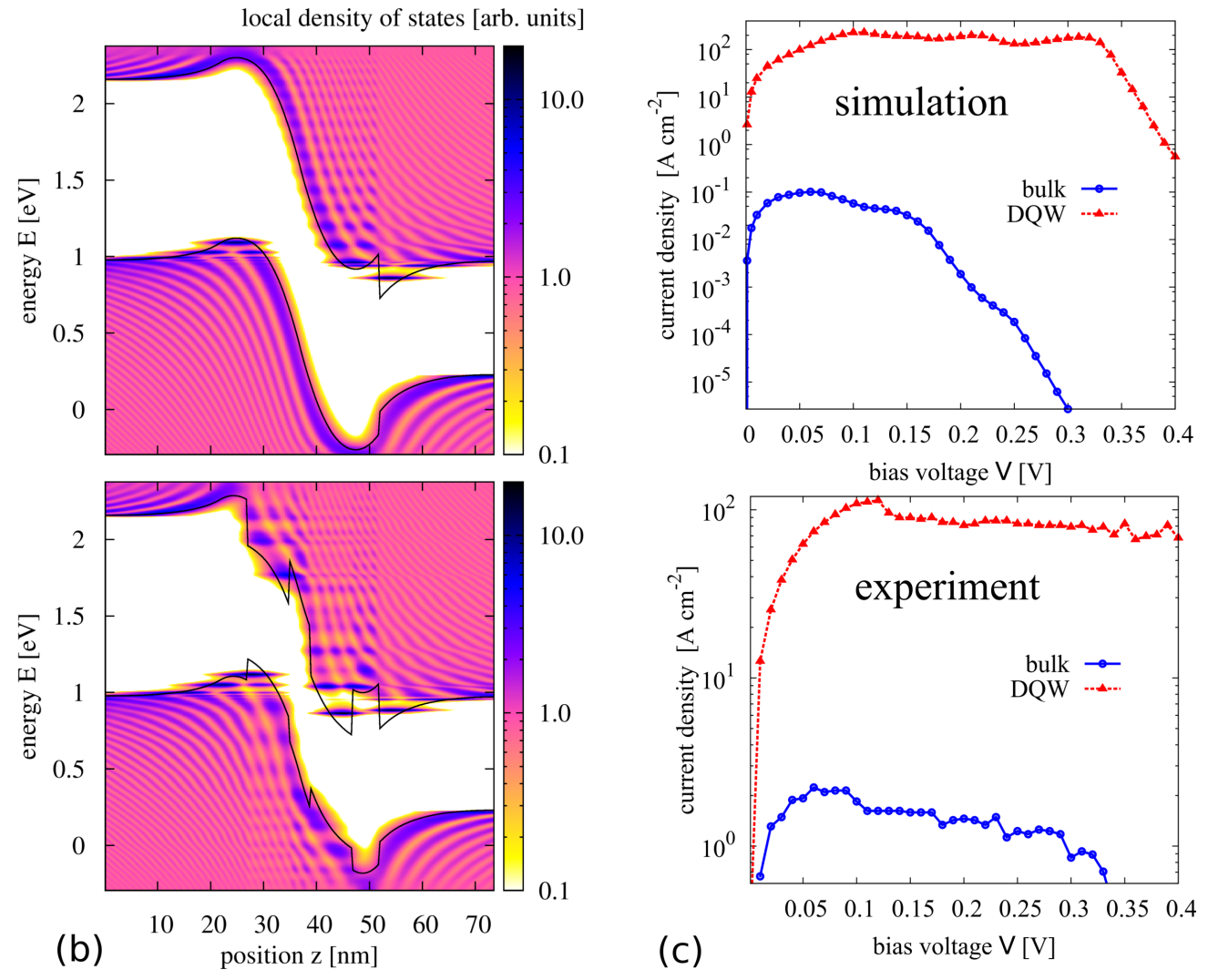

Figure 14. (color online) Structure and characteristics of bulk and DQW tunnel-heterojunction devices investigated experimentally

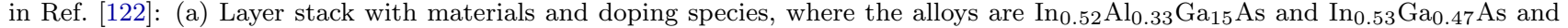
the doping densities amount to $2 \times 10^{17} \mathrm{~cm}^{-3}$ for $p^{+} / n^{+}$and $10^{19} \mathrm{~cm}^{-3}$ for $p^{++} / n^{++}$. (b) Local density of states in the junction region, exhibiting a large variety of bound and quasi-bound states in the quantum wells induced by the heterogeneous doping and the heterostructure potential. (c) Current-voltage characteristics for the bulk and DQW tunnel junction devices: the simulation reproduces the experimental peak voltage and the large difference in magnitude between the two architectures. The deviation at large bias is due to the onset of prevailing trap-assisted tunneling, which is not included in the model. Adapted with permission from Ref. [33].

extraction occurs. This situation of optical operation in electronically isolated QD is depicted schematically in Fig. 16(a) for the most basic simplification to a twolevel system. Conventionally, the emission spectrum is computed based on the occupation of the QD levels as obtained from a rate equation model based on Fermi-Golden-Rule transition rates for the eigenstates of the isolated QD, and the eigenenergies for the peak position, while the line shape of the absorption and emission is added using empirical expressions for homogeneous and inhomogeneous broadening. Using the NEGF formalism, physical line shapes are provided automatically and in relation to the actual relaxation processes, such as due to electron-phonon interaction or Auger-cooling [127, 128].

In order to enable full spectrum computations by NEGF, the single QD Hamiltonian is parametrized using the microscopic solution of the 3-D QD Schrödinger equation. The electronic single particle states of the QD ("QD orbitals") are thereby described via the field operator

$$
\hat{\Psi}(\mathbf{r}, t)=\sum_{n} \psi_{n}(\mathbf{r}) \hat{d}_{n}(t)
$$

with $\psi$ the single electron wave functions and $\hat{d}_{n}$ the annihilation operator for electrons in QD orbitals $n$. The non-equilibrium quantum statistical averages of these field operators define the electronic single particle Green's functions,

$$
G_{m n}\left(t, t^{\prime}\right)=-\frac{i}{\hbar}\left\langle\hat{T}_{C}\left\{\hat{d}_{m}(t) \hat{d}_{n}^{\dagger}(t)\right\}\right\rangle
$$

In order to obtain the photoluminescent emission spectrum, the NEGF equations (38) and (39) are solved in the presence of electron-photon interaction (for the polarization build-up) as well as electron-phonon and electron-electron interaction (for relaxation of photogenerated carriers), and with vanishing contact selfenergies (closed contacts $\equiv$ infinite potential barriers). 
(a)
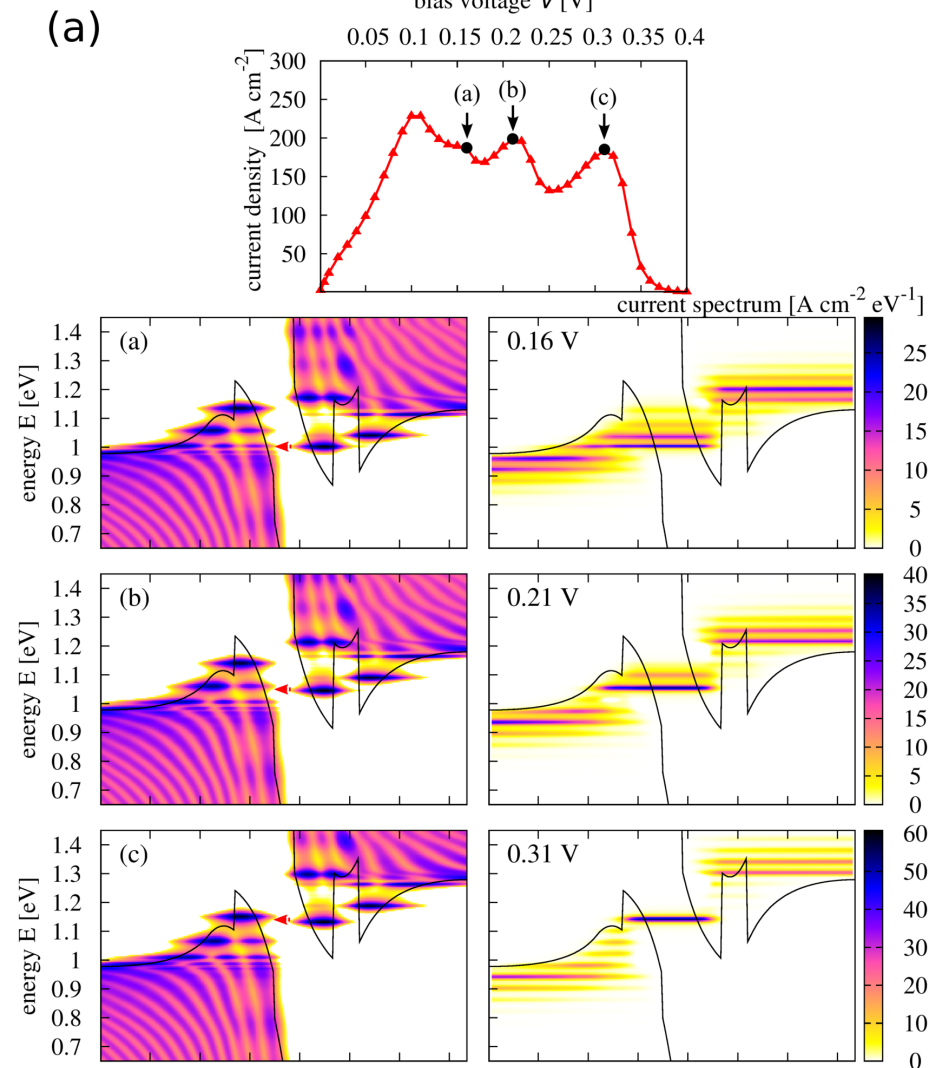

$\begin{array}{lllllll}10 & 20 & 30 & 40 & 50 & 60 & 70 \\ \text { position } z[n m]\end{array}$

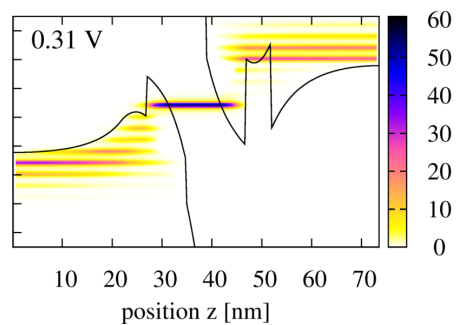

(b)

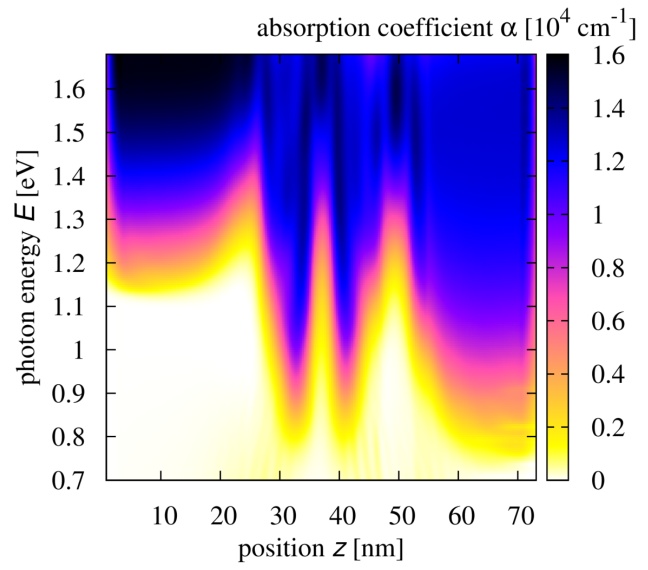

(c)

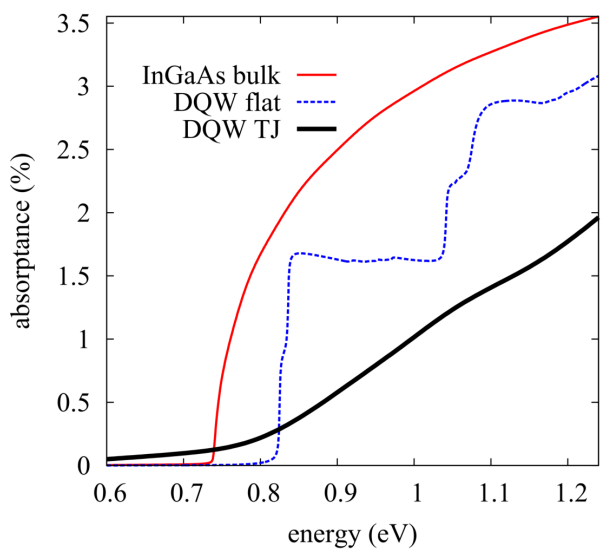

Figure 15. (color online) (a) Microscopic resolution of resonance features in the current-voltage characteristics of the DQW TJ by relation to the alignment of localized states in the junction region (left panel). The associated spectral current flow (right panel) reflects the crucial role of inelastic scattering by coupling of electrons to optical phonons with discrete energy. (b) Spatially resolved absorption coefficient of the DQW TJ, displaying the band-tailing effects of the strong doping-induced fields in the central region. (c) The absorptance of the DQW TJ (thick solid line) is reduced as compared to the bulk QW material (thin solid line), but deviates strongly from the absorptance of a DQW structure at flat band conditions (dashed line). Adapted with permission from Ref. [33], and from Ref. [123].

In the basis (75), the scattering components of the selfenergy for the stimulated electron-photon processes reads

$$
\begin{aligned}
\Sigma_{m n}^{e \gamma, \lessgtr}(E, \hbar \omega)= & \sum_{k, l} \mathcal{M}_{m k}^{e \gamma, \eta} \mathcal{M}_{l n}^{e \gamma, \eta}\left\{G_{k l}^{\lessgtr}(E-\hbar \omega)\right. \\
& \left.+G_{k l}^{\lessgtr}(E+\hbar \omega)\right\} A_{\eta}^{2}(\hbar \omega),
\end{aligned}
$$

where the coupling matrix elements are defined as

$$
\mathcal{M}_{m n}^{e \gamma, \alpha} \equiv\left(\frac{e}{m_{0}}\right) \mathrm{p}_{m n}^{\alpha} .
$$

Similarly, the self-energy components for isotropic spontaneous emission into free-field modes read

$\bar{\Sigma}_{m n}^{e \gamma, \lessgtr}(E) \approx \frac{\mu_{0}}{\pi c_{0}} \sum_{k, l} \overline{\mathcal{M}}_{m k}^{e \gamma} \overline{\mathcal{M}}_{l n}^{e \gamma} \int_{0}^{\infty} \frac{d E^{\prime}}{2 \pi \hbar} E^{\prime} G_{k l}^{\lessgtr}\left(E-E^{\prime}\right)$.
For the scattering with phonons, only interaction with equilibrium bulk modes is considered [129], which results in the self-energy expressions

$$
\begin{aligned}
\Sigma_{n m}^{e p, \lessgtr}(E)= & \sum_{\Lambda, \mathbf{Q}} \sum_{k, l} \mathcal{M}_{n k}^{e p}(\Lambda, \mathbf{Q}) \mathcal{M}_{m l}^{e p, *}(\Lambda, \mathbf{Q}) \\
& \times\left[N_{\Lambda \mathbf{Q}} G_{k l}^{\lessgtr}\left(E \mp \hbar \Omega_{\Lambda \mathbf{Q}}\right)\right. \\
& \left.+\left(N_{\Lambda \mathbf{Q}}+1\right) G_{k l}^{\lessgtr}\left(E \pm \hbar \Omega_{\Lambda \mathbf{Q}}\right)\right]
\end{aligned}
$$

where

$$
\mathcal{M}_{l m}^{e p}(\Lambda, \mathbf{Q})=\frac{1}{\sqrt{V}} U_{\Lambda, \mathbf{Q}} \int d^{3} r \psi_{l}^{*}(\mathbf{r}) \psi_{m}(\mathbf{r}) e^{i \mathbf{Q} \cdot \mathbf{r}} .
$$

The form assumed for the interaction potential is the same as for the planar case, i.e., given by Eqs. (62) and (64). 
(a)

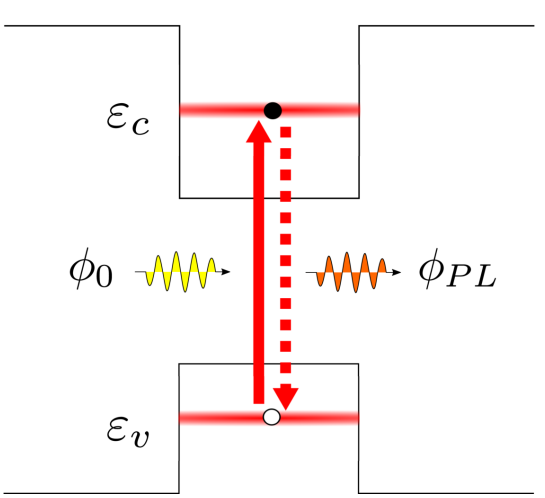

(b)

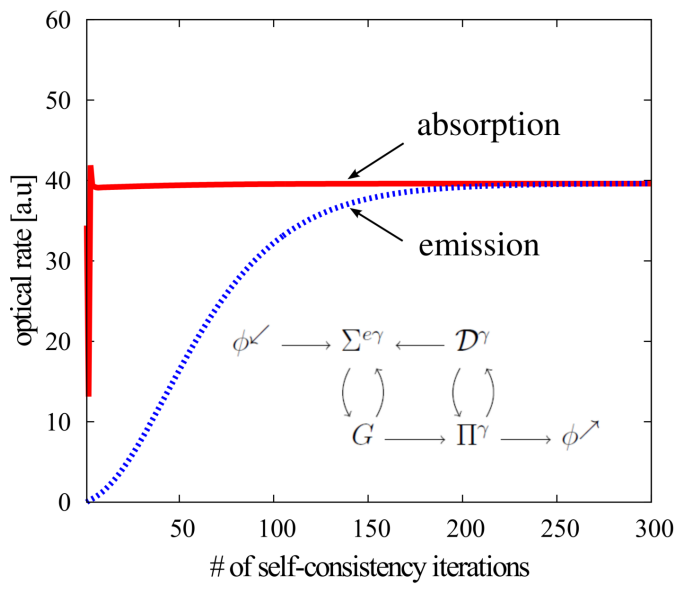

Figure 16. (a) Schematic representation of the model system for QD fluorescence, where the incident photon flux $\phi_{0}$ generates a polarization of the system that leads to spontaneous emission (flux $\left.\phi_{\mathrm{PL}}\right)$. (b) The evolution of the system towards the photoluminescent steady state is reflected in the convergence of absorption and emission rates in the self-consistency iteration of the carrier Green's functions and the electron-photon interaction self-energies. Reproduced from Ref. [125] with permission from the PCCP Owner Societies.

The single QD problem is the only case so far where we included a treatment of electron-electron interaction. In the QD orbital basis, the direct-collision self-energy acquires the form

$$
\begin{aligned}
\Sigma_{n m}^{d c, \lessgtr}(E)= & i \hbar \sum_{n_{1}, n_{2}, n_{3}} \sum_{m_{1}, m_{2}, m_{3}} V_{m_{1} m_{2} n_{3} m} V_{m_{3} n n_{1} n_{2}} \\
& \times \int \frac{d E^{\prime}}{2 \pi \hbar} \pi \pi_{n_{2} m_{2} n_{3} m_{3}}^{\lessgtr}\left(E^{\prime}\right) G_{n_{1} m_{1}}^{\lessgtr}\left(E-E^{\prime}\right)
\end{aligned}
$$

with the Coulomb matrix elements

$V_{n k l m}=\int d^{3} r \int d^{3} r^{\prime} \psi_{n}^{*}(\mathbf{r}) \psi_{k}^{*}\left(\mathbf{r}^{\prime}\right) V\left(\mathbf{r}-\mathbf{r}^{\prime}\right) \psi_{l}\left(\mathbf{r}^{\prime}\right) \psi_{m}(\mathbf{r})$,

where $V(\mathbf{r})=e^{2} /\left(4 \pi \varepsilon_{0} \varepsilon(\mathbf{r})|\mathbf{r}|\right)$ is the (bare) Coulomb potential, and the longitudinal intraband polarization functions are given by

$$
\pi_{k l m n}^{\lessgtr}(E)=-i \hbar \int \frac{d E^{\prime}}{2 \pi \hbar} G_{k l}^{\lessgtr}\left(E^{\prime}\right) G_{m n}^{\gtrless}\left(E^{\prime}-E\right),
$$

where all indices denote states of the same carrier species.

Since photogenerated carriers cannot escape to contacts, polarization starts to build up, and with it the emission. The relation between the polarization function and the absorption and emission rates is given by the respective terms in Eq. (49) for the net radiative rate: in the $\mathrm{QD}$ orbital bases, the (net) absorption rate reads

$$
\mathcal{R}_{\text {abs,net }}(\hbar \omega)=\sum_{\eta} \Phi_{0 \eta}^{\gamma}(\hbar \omega) \mathcal{A}_{\eta}(\hbar \omega)
$$

where $\Phi_{0}^{\gamma}$ is the incident photon flux and the absorption cross-section is given by

$$
\mathcal{A}_{\eta}(\hbar \omega)=\frac{c_{0}}{2 n_{r} \omega} \Re\left\{i \hat{\Pi}_{\eta \eta}(\hbar \omega)\right\} .
$$

The photon self-energy term $\hat{\Pi} \equiv \Pi^{>}-\Pi^{<}$is defined via the components

$$
\Pi_{\eta \eta^{\prime}}^{\lessgtr}(E)=\mu_{0} \sum_{m k l n} \mathcal{M}_{m k}^{e \gamma, \eta} \mathcal{M}_{l n}^{e \gamma, \eta^{\prime}} \mathcal{P}_{m k l n}^{\lessgtr}(E) .
$$

The electron-hole polarization function elements have a form similar to the intra-band terms (84),

$$
\mathcal{P}_{m k l n}^{\lessgtr}(E)=-i \hbar \int \frac{d E^{\prime}}{2 \pi \hbar} G_{m k}^{\lessgtr}\left(E^{\prime}\right) G_{l n}^{\gtrless}\left(E^{\prime}-E\right),
$$

but where $(m, k)$ label electron states and $(l, n)$ label hole states, and which is related to the spectral function $\hat{\mathcal{P}} /(2 \pi)$ of occupied electron-hole pairs $(\hat{\mathcal{P}} \equiv$ $i\left\{\mathcal{P}^{>}-\mathcal{P}^{<}\right\}$) [83]. Similarly, the spectral rate of spontaneous emission into an optically isotropic medium can be written as follows:

$$
\overline{\mathcal{R}}_{\mathrm{em}}(\hbar \omega)=\frac{n_{r} \omega}{2 \pi^{2} \hbar c_{0}} \Re\left\{i \bar{\Pi}^{<}(\hbar \omega)\right\} .
$$

Figure 16(b) displays the evolution of the absorption and emission rates of the two-level model under resonant optical excitation during the self-consistency iteration of Green's functions and self-energies. Upon reaching convergence, the two rates are balanced, which amounts to the steady state of photoluminescent device operation.

The formalism is applied to the fluorescence of $\mathrm{PbSe} / \mathrm{PbS}$ core-shell QD ensembles under nearinfrared (NIR) illumination as used for advanced QDsensitized up-conversion devices [125]. The electronic structure of the QD is computed based on semiempirical methods, such as $\mathbf{k} \cdot \mathbf{p}$ theory $[130,131]$ or the empirical pseudo-potential method (EPM) [132]. 


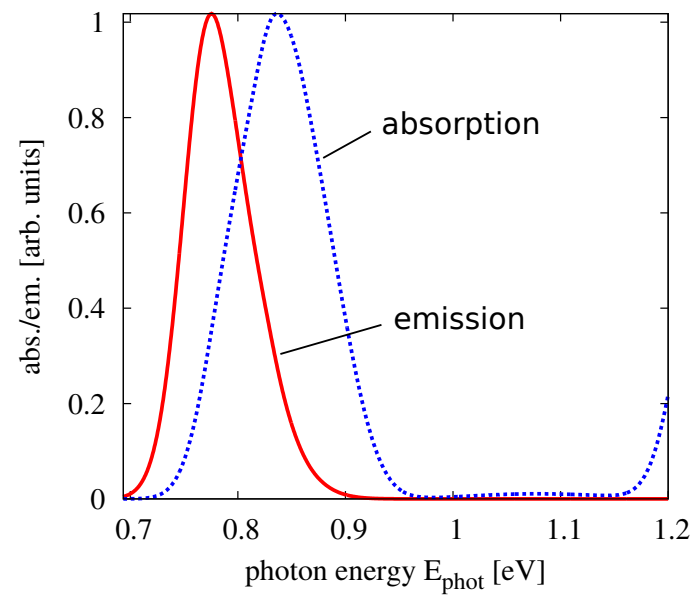

Figure 17. Absorption (dotted) and emission spectra of a single PbSe QD of $3 \mathrm{~nm}$ radius under near-infrared solar illumination. The excited-state relaxation via electron-phonon interaction and Auger electron cooling results in sizable line broadening and a pronounced Stokes shift.

Figure 17 shows the absorption and emission spectra of a single PbSe QD $(\mathrm{R}=3 \mathrm{~nm})$ with electronic structure from an EPM model and under illumination with full solar NIR spectrum between $0.76 \mathrm{eV}$ and $1.1 \mathrm{eV}$. Emission is into an optically isotropic medium and excited state relaxation is mediated via electronphonon (bulk AC and LO) coupling and Auger electron cooling. The relaxation processes result in sizable line broadening and Stokes shift, which are not present in the spectra resulting from the semiclassical rate equation models.

5.3.2. Quantum dot array architectures In photovoltaics, quantum dot arrays are mainly used as tunable absorbers in multi-junction devices $[133,134]$ and for intermediate band solar cells [135]. In difference to their quantum well counterparts discussed above, the confinement in all spatial dimensions renders the full microscopic resolution of the generation and transport problem prohibitively expensive from the computational point of view. For this reason, we extend the parametrization scheme of the single QD (SQD) case to 1-D QD superlattice (QDSL) structures with finite number of periods and furnished with carrier selective contacts. The only element that is new with respect to the SQD model - in addition to the contacts - are the interdot hopping terms, which are obtained from the transfer integrals of the localized basis elements in the form of QD orbitals or Wannier functions. This amounts to field operators of the form

$$
\hat{\Psi}(\mathbf{r}, t)=\sum_{n, L} \psi_{n, L}(\mathbf{r}) \hat{d}_{n, L}(t),
$$

with $\hat{d}_{n, L}$ the annihilation operator for electrons in QD orbitals $n$ at $\mathrm{QD}$ site $L$, and $\psi_{n, L}$ the corresponding QD orbital wave function. In this basis, the model corresponds to a simple nearest-neighbor tight-binding chain of finite length expressed by the Hamiltonian

$$
\begin{aligned}
\hat{\mathcal{H}}_{0}^{m}= & \sum_{L=1}^{N_{\mathrm{QD}}-1} t_{m, L L+1}\left[\hat{d}_{m, L+1}^{\dagger} \hat{d}_{m, L}+h . c .\right] \\
& +\sum_{L=1}^{N_{\mathrm{QD}}} \varepsilon_{m, L} \hat{n}_{m, L},
\end{aligned}
$$

where $N_{\mathrm{QD}}$ is the number of dots, $t_{m}$ is the inter-dot coupling, $\hat{n}_{m} \equiv \hat{d}_{m}^{\dagger} \hat{d}_{m}$ is the carrier density operator and $\varepsilon_{m}$ is the QD energy level. The dot-contact coupling is considered in the usual way by means of a contact self-energy that vanishes for closed contacts. Use of the basis in (90) introduces an additional spatial index in the expressions for Green's functions and interaction self-energies as defined above for the single QD case. Due to the localized nature of the basis functions, the coupling matrices (78) and (81) are approximated as diagonal in the spatial index.

Figure 18 (a) shows a schematic representation of the above model for the selectively contacted QD array. The corresponding local and integrated density of states as obtained from the Green's functions is displayed in Fig. 18(b). The selective contacts induce a broadening that reflects the hybridization of QD array states with the modes of the bulk electrodes. In the case of QD absorbers with generation and extraction of carriers in QDSL miniband states, the main questions to be addressed are related to the impact on the photovoltaic performance of configurational parameters such as the coupling $t$ between dots and the coupling $V$ from the QD to the contacts, as well as the effects of the finite size $N$. In Fig. 19, the different types of steady-state operation in function of coupling parameters and carrier lifetime are illustrated in terms of the convergence of radiative rates and terminal current: (a) Photoluminescence at vanishing contact coupling (closed contact) corresponding to the situation displayed in Fig. 16. (b) Unit photocarrier extraction efficiency at long lifetime and strong contact coupling. (c) Intermediate regime with sub-unit extraction efficiency, where the emission rate and the rate of carrier extraction sum up to the generation rate. In (d), the extraction efficiency is shown as a function of interdot and dot-contact coupling strength and for different carrier lifetime, revealing the dominant role of the dot-contact coupling [30].

For the application in intermediate band solar cells (IBSC), the selective contacting scheme of the QD array needs to be modified in order to isolate electronically the intermediate band, which 
(a)

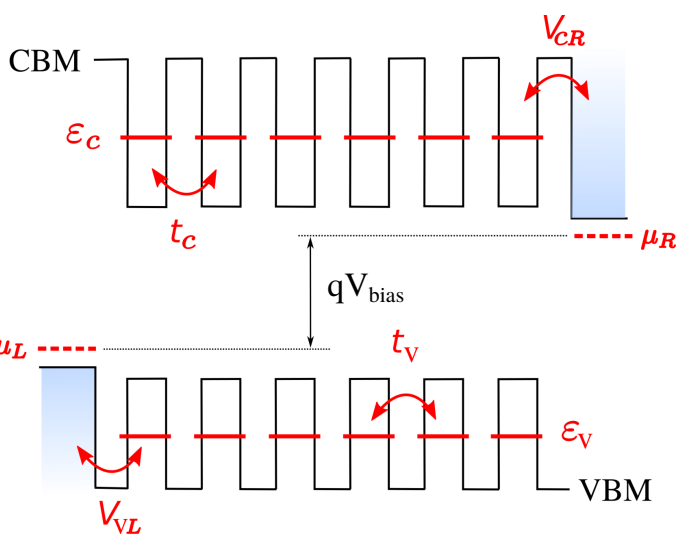

(b)

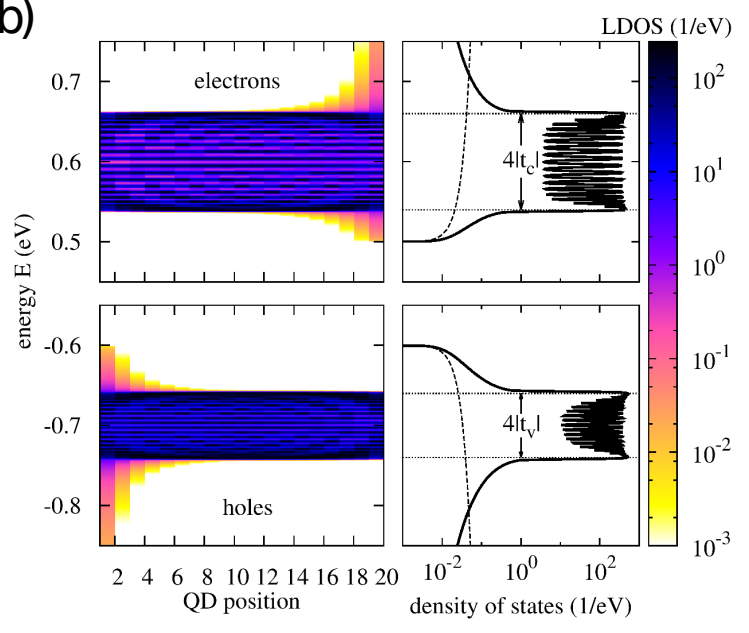

Figure 18. (a) Schematic representation of the effective model for a selectively contacted QD array, with QD energy levels $\varepsilon$, inter-dot hopping parameters $t$ and dot-contact coupling $V$. Again, a finite voltage between contacts is imposed by a corresponding splitting of the chemical potentials $\Delta \mu=q V_{\text {bias }}$. (b) Local and integrated density of states, illustrating the hybridization of QD and electrode states at the majority carrier contacts. Adapted with permission from Ref. [30].

amounts to closing also the majority carrier contacts for the lowest miniband. Figure 20(a) displays the modified QD-IBSC setup including the optical transitions to be considered, i.e., two-step transitions via the intermediate band utilizing lower-energy photons in addition to the direct transitions between the contacted minibands. The corresponding local and integrated DOS is shown in Fig. 20(b) and reflects the isolation of the lower electron miniband, where the electronic states are broadened solely due to the interaction with phonons. The lack of contact broadening represents a challenge for the convergence of the optoelectronic rates in the NEGF self-consistency iteration: as shown in Fig. 21(a), in the first phase of the iteration process, the DOS needs to converge, before the actual convergence of the rates for interband- and subband transitions sets (a)

(b)
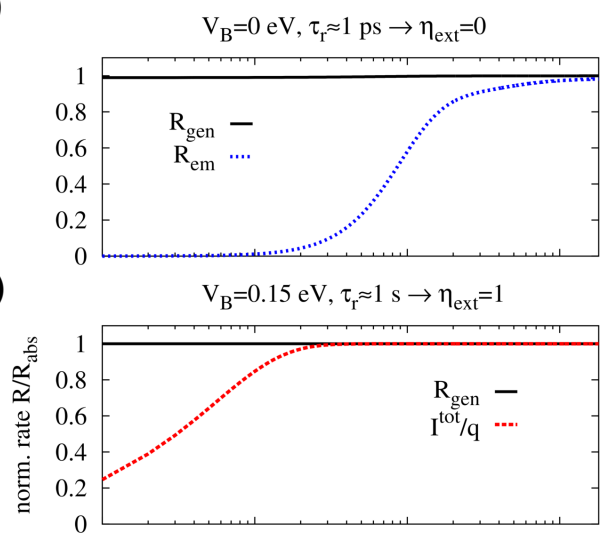

(c)

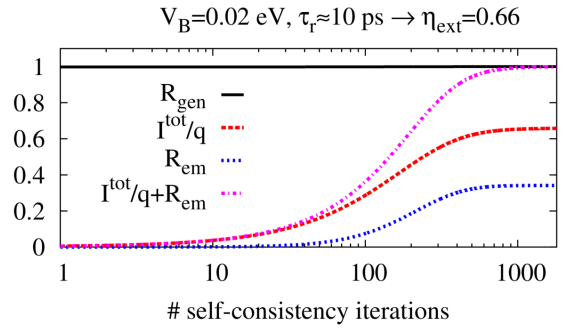

(d)

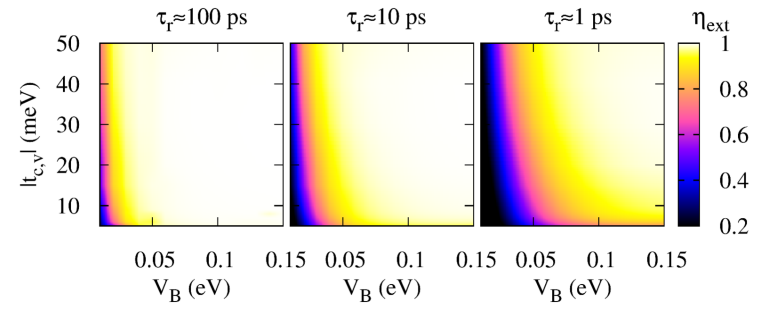

Figure 19. Photocarrier extraction at short circuit as a function of dot-contact coupling $V_{\mathrm{B}}$ and carrier lifetime $\tau_{r}$ for fixed (ac) or varying (d) interdot-coupling $t$ : (a) At closed contacts, all of the photogenerated carriers recombine radiatively. (b) At large contact coupling and long carrier lifetime, all of the photogenerated carriers are extracted as photocurrent. (c) At intermediate contact coupling and moderate carrier lifetime, photocarrier extraction is incomplete. (d) At all values of the carrier lifetime, the extraction efficiency is dominated by the dot-contact coupling. Adapted with permission from Ref. [30].

in. While the rate for the interband transition $\left(R_{\mathrm{v} \rightarrow \mathrm{C}_{1}}\right)$ remains almost uniform, the rate for the subband transition $\left(R_{\mathrm{c}_{1} \rightarrow \mathrm{c}_{2}}\right)$ requires accumulation of photogenerated charge carriers in the lower miniband in order to evolve to the common steady-state value. This is reflected in the convergence behaviour of the photocurrent $J_{\mathrm{sc}}$ extracted at the selective contacts. The non-radiative decoupling of the electron minibands leads to radiative dark current-voltage characteristics that - in the bias region close to the open circuit voltage - are no longer dominated by the radiative currents of the lower or higher minibands in single junction configuration, but by the two-step process exhibiting a much larger ideality factor, as shown in Fig. 21(b). 
(a)

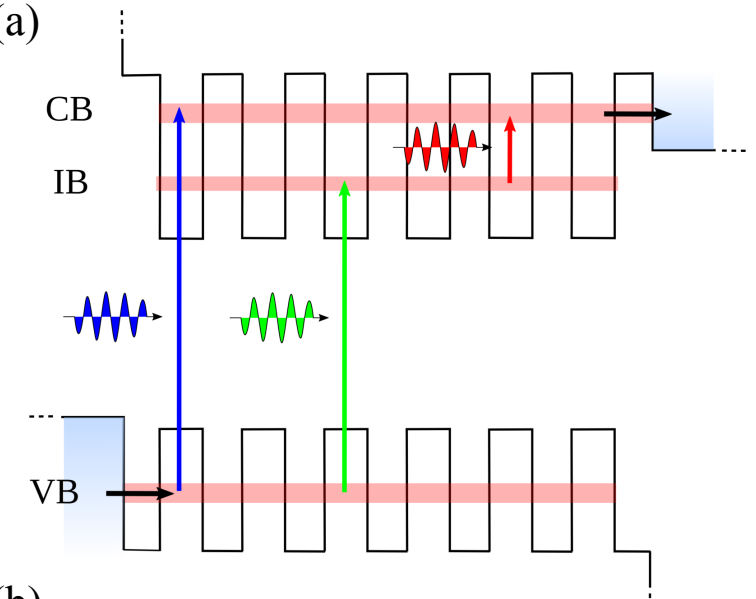

(b)

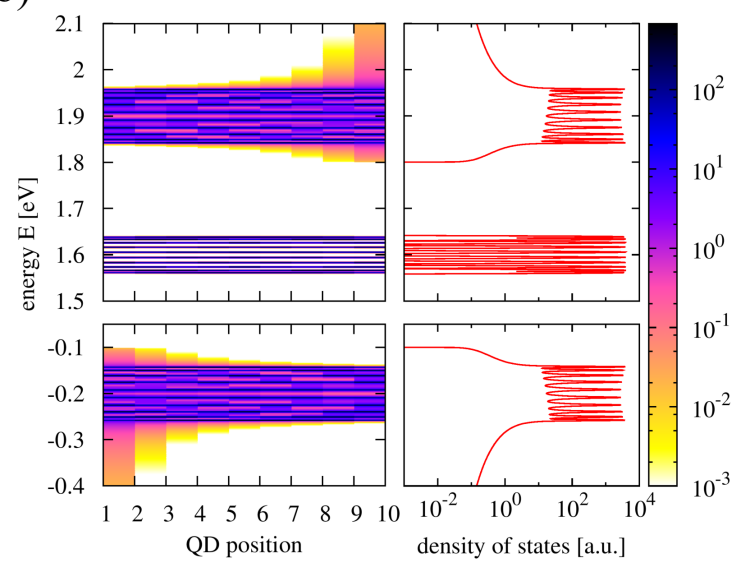

Figure 20. (a) Schematic representation of the effective model for QD-IBSC, based on a selectively contacted QD array with electronically isolated lowest miniband. Adapted with permission from Ref. [68]. (b) Corresponding local and integrated density of states, reflecting the absence of contacts in the intermediate band.

\subsection{Nanowire solar cells}

For the sake of completeness, the case of nanowire solar cells is addressed briefly here, as such devices also count among the nanostructure-based photovoltaic architectures considered in this Topical Review. While nanowire absorbers have been simulated by NEGF [28], in most of the cases, the diameters considered in nanowire photovoltaics for the exploitation of antenna effects are too large to allow for electronic quantum confinement, and the transverse modes are dense enough to be treated as a continuum, which renders the nanowire case very similar to the ultrathin absorber solar cell covered in Sec. 5.1.

\section{Conclusions}

Complex nanostructure architectures are ubiquitous in the field of high efficiency photovoltaics. An accurate and physically valid description of the microscopic pro-

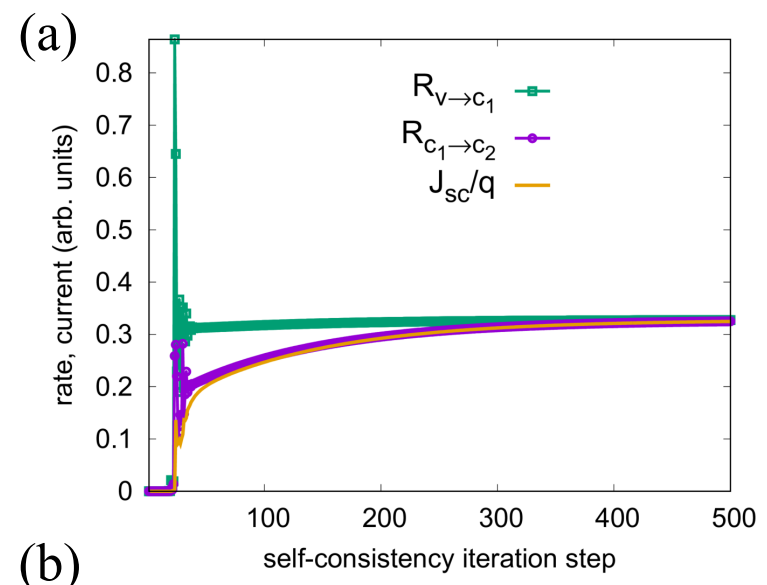

(b)

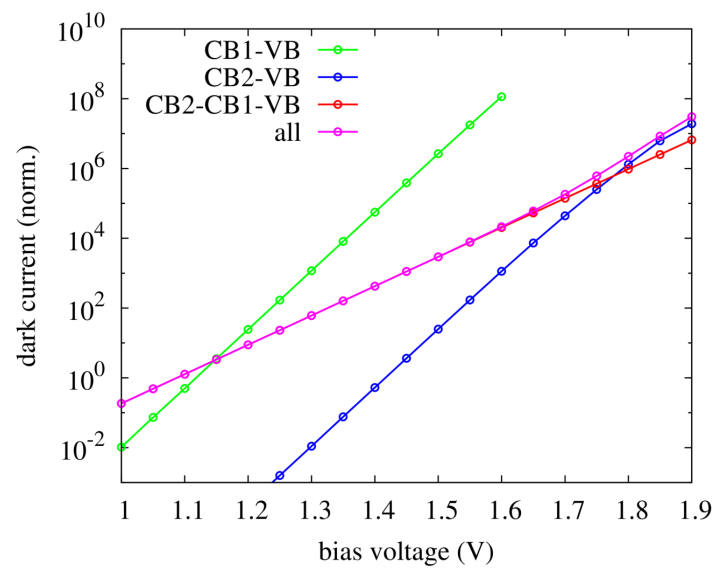

Figure 21. (a) Convergence of the net inter- and subband transition rates for the QD-IBSC configuration during the NEGF self-consistency iteration. In the first phase, the density of states consolidates via broadening due to electron-phonon interaction. The interband transition rate then converges quickly to the steady-state value, while the subband transition rate requires build-up of photogenerated charge carrier population in the lower miniband in order to evolve to the common steady-state rate. This behaviour is reflected in the convergence of the photocurrent extracted at selective contacts. Adapted with permission from Ref. [68]. (b) As a consequence of the nonradiative decoupling of the electron minibands, the radiative dark current-voltage characteristics are no longer dominated by either single-junction characteristics with lower or higher band gaps, but by the two-step process exhibiting much larger ideality factor.

cesses governing the photovoltaic operation of such devices requires a theory and simulation framework that reaches beyond the limitations of the standard semiclassical picture, while reproducing the results of the latter in situation where it is applicable. The quantumkinetic approach presented here provides such a framework and gives insight into the microscopic mechanisms of photovoltaics at the nanoscale. Most importantly, the approach allows to establish the essential structure-property relations that govern the photovoltaic performance of a given device architecture, by directly linking microscopic configuration param- 
eters with mesoscopic device characteristics. At the same time, it provides a valuable instrument for the validation of computationally less expensive but more approximate treatments.

While the formalism is very general and applicable to a wide range of effects relevant to photovoltaic device operation, such as photocurrent generation, electroluminescence and photoluminescence, models still need to be customized to the specific situation in order to allow meaningful computations. However, with the advances in computational power, the enhanced accuracy of the underlying electronic structure and the growing spatial extent of permissible model domains will allow increasingly predictive simulations of nanostructure solar cell characteristics.

\section{Acknowledgements}

This work has benefited from fruitful discussions within COST action MP1406 - MultiscaleSolar. The author gratefully acknowledges funding from the European Commission Horizon 2020 project No. 676629 ("EoCoE") and computing time granted on the supercomputer JURECA at Jülich Supercomputing Centre (JSC).

\section{References}

[1] K. Yoshikawa, H. Kawasaki, W. Yoshida, T. Irie, K. Konishi, K. Nakano, T. Uto, D. Adachi, M. Kanematsu, H. Uzu, and K. Yamamoto, "Silicon heterojunction solar cell with interdigitated back contacts for a photoconversion efficiency over 26\%," Nature Energy, vol. 2, p. 17032, 2017.

[2] T. Tiedje, E. Yablonovitch, G. Cody, and B. Brooks, "Limiting efficiency of silicon solar cells," IEEE Trans. Electron Devices, vol. 31, pp. 711-716, 1984.

[3] M. A. Green, "Third generation photovoltaics: Ultra-high conversion efficiency at low cost," Prog. Photovolt: Res. Appl., vol. 9, p. 123, 2001.

[4] A. Martí, Next generation photovoltaics : high efficiency through full spectrum utilization / ed. by Antonio Marti Series in optics and optoelectronics, Bristol: IOP, 2004.

[5] M. A. Green, Third Generation Photovoltaics Advanced Solar Energy Conversion. Springer-Verlag, Berlin Heidelberg, 2006.

[6] M. A. Green, "Potential for low dimensional structures in photovoltaics," Mater. Sci. Eng., B, vol. 74, no. 1-3, pp. $118-124,2000$.

[7] G. Conibeer, D. König, M. Green, and J. Guillemoles, "Slowing of carrier cooling in hot carrier solar cells," Thin Solid Films, vol. 516, no. 20, pp. 6948 - 6953, 2008.

[8] A. Martí, L. Cuadra, and A. Luque, "Partial filling of a quantum dot intermediate band for solar cells," IEEE Trans. Electron Devices, vol. 48, pp. 2394-2399, 2001.

[9] K. Barnham and G. Duggan, "A new approach to highefficiency multi-band-gap solar cells," J. Appl. Phys., vol. 67, pp. 3490-3493, 1990.

[10] V. Aroutiounian, S. Petrosyan, A. Khachatryan, and K. Touryan, "Quantum dot solar cells," J. Appl. Phys., vol. 89, no. 4, p. 2268, 2001.
[11] U. Aeberhard, "Simulation of nanostructure-based highefficiency solar cells: Challenges, existing approaches, and future directions," IEEE J. Sel. Top. Quantum Electron., vol. 19, no. 5, p. 4000411, 2013.

[12] G. Araújo, A. Martí, F. Ragay, and J. Wolter, "Efficiency of multiple quantum well solar cells," in Proc. 12th European Photovoltaic Solar Energy Conference, p. $1481,1994$.

[13] P. Würfel, "The chemical potential of radiation," J. Phys. C: Solid State Phys., vol. 15, p. 3967, 1982.

[14] U. Rau, "Reciprocity relation between photovoltaic quantum efficiency and electroluminescent emission of solar cells," Phys. Rev. B, vol. 76, no. 8, p. 085303, 2007.

[15] U. Aeberhard and U. Rau, "Microscopic perspective on photovoltaic reciprocity in ultrathin solar cells," Phys. Rev. Lett., vol. 118, p. 247702, 2017.

[16] S. M. Ramey and R. Khoie, "Modeling of multiplequantum-well solar cells including capture, escape, and recombination of photoexcited carriers in quantum wells," IEEE Trans. Electron Devices, vol. 50, p. 1179, 2003.

[17] M. Gioannini, A. P. Cedola, N. D. Santo, F. Bertazzi, and F. Cappelluti, "Simulation of Quantum Dot Solar Cells Including Carrier Intersubband Dynamics and Transport," IEEE J. Photovolt., vol. 3, pp. 1271-1278, 2013.

[18] L. E. Henrickson, "Nonequilibrium photocurrent modeling in resonant tunneling photodetectors," J. Appl. Phys, vol. 91, p. 6273, 2002.

[19] M. A. Naser, M. J. Deen, and D. A. Thompson, "Spectral function and responsivity of resonant tunneling and superlattice quantum dot infrared photodetectors using green's function," J. Appl. Phys., vol. 102, no. 8, p. 083108, 2007.

[20] M. F. Pereira and K. Henneberger, "Microscopic theory for the influence of Coulomb correlations in the lightemission properties of semiconductor quantum wells," Phys. Rev. B, vol. 58, pp. 2064-2076, 1998.

[21] S.-C. Lee and A. Wacker, "Nonequilibrium Green's function theory for transport and gain properties of quantum cascade structures," Phys. Rev. B, vol. 66, p. 245314, 2002.

[22] T. Kubis, C. Yeh, P. Vogl, A. Benz, G. Fasching, and C. Deutsch, "Theory of nonequilibrium quantum transport and energy dissipation in terahertz quantum cascade lasers," Phys. Rev. B, vol. 79, p. 195323, 2009.

[23] S. Steiger, R. G. Veprek, and B. Witzigmann, "Electroluminescence from a quantum-well LED using NEGF," in Proceedings 13th International Workshop on Computational Electronics, 2009.

[24] D. A. Stewart and F. Leonard, "Energy conversion efficiency in nanotube optoelectronics," Nano Lett., vol. 5, p. 219, 2005.

[25] U. Aeberhard and R. H. Morf, "Microscopic nonequilibrium theory of quantum well solar cells," Phys. Rev. B, vol. 77, p. 125343, 2008.

[26] U. Aeberhard, "Theory and simulation of photogeneration and transport in $\mathrm{Si}-\mathrm{SiOx}$ superlattice absorbers," Nanoscale Res. Lett., vol. 6, p. 242, 2011.

[27] U. Aeberhard, A. Gonzalo, and J. M. Ulloa, "Photocarrier extraction in GaAsSb/GaAsN type-II QW superlattice solar cells," Appl. Phys. Lett., vol. 112, no. 21, p. $213904,2018$.

[28] A. Buin, A. Verma, and S. Saini, "Optoelectronic response calculations in the framework of k.p coupled to non-equilibrium Green's functions for one-dimensional systems in the ballistic limit," J. Appl. Phys., vol. 114, no. 3, p. 033111, 2013. 
[29] U. Aeberhard, "Effective microscopic theory of quantum dot superlattice solar cells," Opt. Quantum. Electron., vol. 44, pp. 133-140, 2012.

[30] A. Berbezier and U. Aeberhard, "Impact of nanostructure configuration on the photovoltaic performance of quantum-dot arrays," Phys. Rev. Applied, vol. 4, p. 044008, 2015.

[31] N. Cavassilas, C. Gelly, F. Michelini, and M. Bescond, "Reflective Barrier Optimization in Ultrathin SingleJunction GaAs Solar Cell," IEEE J. Photovolt., vol. 5, no. 6, pp. 1621-1625, 2015.

[32] U. Aeberhard, "Simulation of ultrathin solar cells beyond the limits of the semiclassical bulk picture," IEEE $J$. Photovolt., vol. 6, no. 3, pp. 654-660, 2016.

[33] U. Aeberhard, "Theoretical investigation of direct and phonon-assisted tunneling currents in InAlGaAs/InGaAs bulk and quantum-well interband tunnel junctions for multijunction solar cells," Phys. Rev. B, vol. 87, p. 081302, 2013.

[34] U. Aeberhard, "Theory and simulation of quantum photovoltaic devices based on the non-equilibrium Greens function formalism," J. Comput. Electron., vol. 10, pp. 394-413, 2011

[35] R. Rhyner and M. Luisier, "Atomistic modeling of coupled electron-phonon transport in nanowire transistors," Phys. Rev. B, vol. 89, p. 235311, 2014.

[36] L. P. Kadanoff and G. Baym, Quantum Statistical Mechanics. Benjamin, Reading, Mass., 1962.

[37] L. Keldysh, "Diagram technique for nonequilibrium processes," Sov. Phys. JETP, vol. 20, pp. 1018-1026, 1965.

[38] W. Schäfer and M. Wegener, Semiconductor Optics and Transport Phenomena. Springer, Berlin, 2002.

[39] K. Henneberger and H. Haug, "Nonlinear optics and transport in laser-excited semiconductors," Phys. Rev. B, vol. 38, pp. 9759-9770, 1988.

[40] M. F. Pereira and K. Henneberger, "Green's function theory for semiconductor-quantum-well laser spectra," Phys. Rev. B, vol. 53, pp. 16485-16496, 1996.

[41] F. Jahnke and S. W. Koch, "Many-body theory for semiconductor microcavity lasers," Phys. Rev. A, vol. 52, pp. 1712-1727, 1995.

[42] U. Aeberhard, "Quantum-kinetic theory of steady-state photocurrent generation in thin films: Coherent versus incoherent coupling," Phys. Rev. B, vol. 89, p. 115303, 2014.

[43] U. Aeberhard, "Nonequilibrium Green's function theory of coherent excitonic effects in the photocurrent response of semiconductor nanostructures," Phys. Rev. $B$, vol. 86, p. 115317, 2012.

[44] R. Lake, G. Klimeck, R. Bowen, and D. Jovanovic, "Single and multiband modelling of quantum electron transport through layered semiconductor devices," J. Appl. Phys., vol. 81, p. $7845,1997$.

[45] S. Datta, Electronic Transport in Mesoscopic Systems. Cambridge University Press, 1995.

[46] S. Datta, B. M. Arora, and S. Kumar, "Bound exciton effect and carrier escape mechanisms in temperaturedependent surface photovoltage spectroscopy of a single quantum well," Phys. Rev. B, vol. 62, pp. 13604-13611, 2000.

[47] S. Datta, Quantum Transport. Cambridge University Press, 2005.

[48] F. Guinea, C. Tejedor, F. Flores, and E. Louis, "Effective two-dimensional hamiltonian at surfaces," Phys. Rev. $B$, vol. 28, pp. 4397-4402, 1983.

[49] M. P. L. Sancho, J. M. L. Sancho, and J. Rubio, "Quick iterative scheme for the calculation of transfer matrices: application to Mo (100)," J. Phys. F: Met. Phys., vol. 14 , no. 5, pp. 1205-1215, 1984.
[50] A. Umerski, "Closed-form solutions to surface Green's functions," Phys. Rev. B, vol. 55, pp. 5266-5275, 1997.

[51] D. H. Lee and J. D. Joannopoulos, "Simple scheme for surface-band calculations. II. the Green's function," Phys. Rev. B, vol. 23, pp. 4997-5004, 1981.

[52] Y.-C. Chang and J. Schulman, "Complex bandstructure of crystalline solids: an eigenvalue method," Phys. Rev. $B$, vol. 25 , p. $3975,1982$.

[53] R. Bowen, W. Frensley, G. Klimeck, and R. Lake, "Transmission zeros and resonances in multiband models," Phys. Rev. B, vol. 52, p. 2754, 1995.

[54] M. Ogawa, T. Sugano, R. Tominaga, and T. Miyoshi, "Multi-band simulation of resonant tunneling diodes with scattering effects," Physica B, vol. 272, p. 167, 1999 .

[55] A. I. Rahachou and I. V. Zozoulenko, "Light propagation in finite and infinite photonic crystals: The recursive Green's function technique," Phys. Rev. B, vol. 72, p. 155117,2005

[56] V. R. Velasco, F. Garcia-Moliner, L. Miglio, and L. Colombo, "Phonon calculations in superperiodic structures: The surface Green-function matching approach," Phys. Rev. B, vol. 38, pp. 3172-3179, 1988.

[57] F. Garcia-Moliner and V. R. Velasco, "Theory of incomplete crystals, surfaces, defects, interfaces and layered structures," Prog. Surf. Sci., vol. 21, no. 2, pp. $93-162,1986$.

[58] D. Langreth, "Linear and nonlinear response theory with applications," in Linear and Non-linear Electron Transport in solids, vol. 17, pp. 3-32, 1976.

[59] D. Mozyrsky and I. Martin, "Efficiency of thin film photocells," Opt. Commun., vol. 277, p. 109, 2007.

[60] F. Richter, M. Florian, and K. Henneberger, "Generalized radiation law for excited media in a nonequilibrium steady state," Phys. Rev. B, vol. 78, no. 20, p. 205114, 2008.

[61] K. Henneberger and F. Richter, "Exact property of the nonequilibrium photon Green function for bounded media," Phys. Rev. A, vol. 80, no. 1, p. 013807, 2009.

[62] K. S. Thygesen and A. Rubio, "Conserving GW scheme for nonequilibrium quantum transport in molecular contacts," Phys. Rev. B, vol. 77, no. 11, p. 115333, 2008.

[63] T. Schmielau and J. M. F. Pereira, "Nonequilibrium many body theory for quantum transport in terahertz quantum cascade lasers," Appl. Phys. Lett., vol. 95, no. 23, p. 231111, 2009.

[64] H. Mera, M. Lannoo, C. Li, N. Cavassilas, and M. Bescond, "Inelastic scattering in nanoscale devices: One-shot current-conserving lowest-order approximation," Phys. Rev. B, vol. 86, p. 161404, 2012.

[65] H. Mera, M. Lannoo, N. Cavassilas, and M. Bescond, "Nanoscale device modeling using a conserving analytic continuation technique," Phys. Rev. B, vol. 88, p. $075147,2013$.

[66] Y. Lee, M. Lannoo, N. Cavassilas, M. Luisier, and M. Bescond, "Efficient quantum modeling of inelastic interactions in nanodevices," Phys. Rev. B, vol. 93, p. 205411, 2016

[67] Y. Lee, M. Bescond, N. Cavassilas, D. Logoteta, L. Raymond, M. Lannoo, and M. Luisier, "Quantum treatment of phonon scattering for modeling of threedimensional atomistic transport," Phys. Rev. B, vol. 95 , p. 201412, 2017 .

[68] U. Aeberhard, "The nonequilibrium Green's function picture of inelastic processes in nanostructure photovoltaics," J. Comput. Electron., vol. 15, no. 4, pp. 12191232,2016

[69] M. Luisier, "Atomistic simulation of transport phenomena in nanoelectronic devices," Chem. Soc. Rev., vol. 43, pp. 4357-4367, 2014. 
[70] T. Kubis and P. Vogl, "Self-consistent quantum transport theory: Applications and assessment of approximate models," J. Comp. Electron., vol. 6, p. 183, 2007.

[71] Z. Wang, T. White, and K. Catchpole, "Plasmonic nearfield enhancement for planar ultra-thin photovoltaics," IEEE Photon. J., vol. 5, no. 5, p. 8400608, 2013.

[72] J. M. Llorens, J. Buencuerpo, and P. A. Postigo, "Absorption features of the zero frequency mode in an ultra-thin slab," Appl. Phys. Lett., vol. 105, no. 23, p. 231115,2014

[73] I. Massiot, C. Colin, N. Péré-Laperne, P. Roca i Cabarrocas, C. Sauvan, P. Lalanne, J.-L. Pelouard, and S. Collin, "Nanopatterned front contact for broadband absorption in ultra-thin amorphous silicon solar cells," Appl. Phys. Lett., vol. 101, no. 16, p. 163901, 2012.

[74] I. Massiot, C. Colin, C. Sauvan, P. Lalanne, P. R. i Cabarrocas, J.-L. Pelouard, and S. Collin, "Multiresonant absorption in ultra-thin silicon solar cells with metallic nanowires," Opt. Express, vol. 21, no. S3, pp. A372-A381, 2013.

[75] I. Massiot, N. Vandamme, N. Bardou, C. Dupuis, A. Lemaitre, J.-F. Guillemoles, and S. Collin, "Metal Nanogrid for Broadband Multiresonant LightHarvesting in Ultrathin GaAs Layers," ACS Photonics, vol. 1 , no. 9, pp. 878-884, 2014.

[76] X. Wang, M. Khan, J. Gray, M. Alam, and M. Lundstrom, "Design of GaAs Solar Cells Operating Close to the Shockley-Queisser Limit," IEEE J. Photovolt., vol. 3 no. 2 , pp. $737-744,2013$.

[77] W. Yang, J. Becker, S. Liu, Y.-S. Kuo, J.-J. Li, B. Landini, K. Campman, and Y.-H. Zhang, "Ultra-thin GaAs single-junction solar cells integrated with a reflective back scattering layer," J. Appl. Phys., vol. 115, no. 20, p. 203105, 2014

[78] N. Vandamme, C. Hung-Ling, A. Gaucher, B. Behaghel, A. Lemaitre, A. Cattoni, C. Dupuis, N. Bardou, J.-F. Guillemoles, and S. Collin, "Ultrathin GaAs Solar Cells With a Silver Back Mirror," IEEE J. Photovolt., vol. 5, no. 2, pp. 565-570, 2015.

[79] L. C. Hirst, M. K. Yakes, J. H. Warner, M. F. Bennett, K. J. Schmieder, R. J. Walters, and P. P. Jenkins, "Intrinsic radiation tolerance of ultra-thin GaAs solar cells," Appl. Phys. Lett., vol. 109, no. 3, 2016.

[80] U. Aeberhard, "Impact of built-in fields and contact configuration on the characteristics of ultra-thin GaAs solar cells," Appl. Phys. Lett., vol. 109, no. 3, p. 033906 , 2016.

[81] G. D. Mahan, Many-Particle Physics, 2nd ed. Plenum, New York, 1990.

[82] C. Hamaguchi, Basic Semiconductor Physics. Springer, Berlin, 2001

[83] U. Aeberhard, "Quantum-kinetic theory of photocurrent generation via direct and phonon-mediated optical transitions," Phys. Rev. B, vol. 84, p. 035454, 2011.

[84] U. Aeberhard, "Simulation of nanostructure-based and ultra-thin film solar cell devices beyond the classical picture," J. Photon. Energy, vol. 4, no. 1, p. 042099, 2014.

[85] U. Aeberhard, "Simulation of ultra-thin solar cells beyond the semi-classical bulk picture," in 2016 IEEE 43rd Photovoltaic Specialists Conference (PVSC), pp. 35023505,2016

[86] U. Aeberhard, "Microscopic theory and numerical simulation of quantum well solar cells," in Proceedings of SPIE, vol. 7597, p. 759702, 2010.

[87] K. W. J. Barnham, B. Braun, J. Nelson, M. Paxman, C. Button, J. S. Roberts, and C. T. Foxon, "Shortcircuit current and energy efficiency enhancement in a low-dimensional structure photovoltaic device," Appl. Phys. Lett., vol. 59, no. 1, pp. 135-137, 1991.
[88] K. Barnham, J. Connolly, P. Griffin, G. Haarpaintner, J. Nelson, E. Tsui, A. Zachariou, J. Osborne, C. Button, G. Hill, M. Hopkinson, M. Pate, J. Roberts, and T. Foxon, "Voltage enhancement in quantum well solar cells," J. Appl. Phys., vol. 80, no. 2, pp. 1201-1206, 1996.

[89] T. Kitatani, Y. Yazawa, S. Watahiki, K. Tamura, J. Minemura, and T. Warabisako, "Photocurrent and photoluminescence in InGaAs/GaAs multiple quantum well solar cells," Jpn. J. Appl. Phys., vol. 35, no. 8R, p. 4371, 1996.

[90] F. Ragay, A. Marti, G. Araujo, and J. Wolter, "Experimental analysis of the efficiency of heterostructure GaAs-AlGaAs solar cells," Sol. Energy Mater. Sol. Cells, vol. 40, p. 5, 1996.

[91] K. Barnham, I. Ballard, J. Barnes, J. Connolly, B. K. Paul Griffin, J. Nelson, E. Tsui, and A. Zachariou, "Quantum well solar cells," Appl. Surf. Sci., vol. 113, p. $722,1997$.

[92] O. Y. Raisky, W. B. Wang, R. R. Alfano, C. L. Reynolds Jr., and V. Swaminathan, "Investigation of photoluminescence and photocurrent in InGaAsP/InP strained multiple quantum well heterostructures," $J$. Appl. Phys, vol. 81, p. 339, 1997.

[93] A. Freundlich, A. Fotkatzikis, L. Bhusal, L. Williams, A. Alemu, W. Zhu, J. A. H. Coaquira, A. Feltrin, and G. Radhakrishnan, "III-V dilute nitride-based multiquantum well solar cell," J. Cryst. Growth, vol. 301, pp. 993-996, 2007.

[94] A. Alemu and A. Freundlich, "Opportunities in dilute nitride III-V semiconductors quantum confined p-i-n solar cells for single carrier resonant tunneling," Microelectron. J., vol. 40, pp. 421-423, 2009.

[95] R. Dahal, B. Pantha, J. Li, J. Y. Lin, and H. X. Jiang, "InGaN/GaN multiple quantum well solar cells with long operating wavelengths," Appl. Phys. Lett., vol. 94, p. 063505,2009

[96] I. M. Pryce, D. D. Koleske, A. J. Fischer, and H. A. Atwater, "Plasmonic nanoparticle enhanced photocurrent in GaN/InGaN/GaN quantum well solar cells," Appl. Phys. Lett., vol. 96, p. 153501, 2010.

[97] K. Y. Lai, G. J. Lin, Y.-L. Lai, Y. F. Chen, and J. H. $\mathrm{He}$, "Effect of indium fluctuation on the photovoltaic characteristics of InGaN/GaN multiple quantum well solar cells," Appl. Phys. Lett., vol. 96, p. 081103, 2010.

[98] A. Asgari and K. Khalili, "Temperature dependence of InGaN/GaN multiple quantum well based high efficiency solar cell," Sol. Energy Mater. Sol. Cells, vol. 95, pp. 3124-3129, 2011.

[99] Q. Deng, X. Wang, H. Xiao, C. Wang, H. Yin, H. Chen, Q. Hou, D. Lin, J. Li, Z. Wang, and X. Hou, "An investigation on InxGa1-xN/GaN multiple quantum well solar cells," J. Phys. D Appl. Phys., vol. 44, p. 265103, 2011.

[100] R. M. Farrell, C. J. Neufeld, S. C. Cruz, J. R. Lang, M. Iza, S. Keller, S. Nakamura, S. P. DenBaars, U. K. Mishra, and J. S. Speck, "High quantum efficiency InGaN/GaN multiple quantum well solar cells with spectral response extending out to $520 \mathrm{~nm}, "$ Appl. Phys. Lett., vol. 98, p. 201107, 2011

[101] M. C. Wagener, P. J. Carrington, J. R. Botha, and a. Krier, "Simulation of the enhanced infrared photoresponse of type-II GaSb/GaAs quantum ring solar cells," Appl. Phys. Lett., vol. 103, no. 6, p. 063902, 2013.

[102] L. C. Hirst, M. P. Lumb, J. Abell, C. T. Ellis, J. G. Tischler, I. Vurgaftman, J. R. Meyer, R. J. Walters, and M. González, "Spatially indirect radiative recombination in InAlAsSb grown lattice-matched to InP by molecular beam epitaxy," J. Appl. Phys., vol. 117 , p. $215704,2015$. 
[103] H. Esmaielpour, V. R. Whiteside, J. Tang, S. Vijeyaragunathan, T. D. Mishima, S. Cairns, M. B. Santos, B. Wang, and I. R. Sellers, "Suppression of phonon-mediated hot carrier relaxation in type-II InAs/AlAs $\mathrm{Sb}_{1-x}$ quantum wells: a practical route to hot carrier solar cells," Prog. Photovolt: Res. Appl., vol. 24, pp. 591-599, 2016.

[104] Shihua Huang, "Analysis of Photocurrent Spectra of SiGe/Si Quantum-Well Solar Cell," IEEE Trans. Nanotechnol., vol. 9, pp. 142-148, 2010.

[105] N. J. Ekins-Daukes, J. M. Barnes, K. W. J. Barnham, J. P. Connolly, M. Mazzer, J. C. Clark, R. Grey, G. Hill, M. A. Pate, and J. S. Roberts, "Strained and strain-balanced quantum well devices for high-efficiency tandem solar cells," Sol. Energy Mater. Sol. Cells, vol. 68 , p. 71,2001

[106] T. N. D. Tibbits, I. M. Ballard, K. W. J. Barnham, D. B. Bushnell, N. J. Ekins-Daukes, R. Airey, G. Hill, and J. S. Roberts, "The potential for strain-balanced quantum well solar cells in terrestrial concentrator applications," in Proc. 3rd World Conference on Photovoltaic Energy Conversion, vol. 3, pp. 2718-2721, 2003.

[107] D. B. Bushnell, N. J. Ekins-Daukes, K. W. J. Barnham, J. P. Connolly, J. S. Roberts, G. Hill, R. Airey, and M. Mazzer, "Short-circuit current enhancement in bragg stack multi-quantum-well solar cells for multijunction space cell applications," Sol. Energy Mater. Sol. Cells, vol. 75, pp. PII S0927-0248(02)00172-1, 2003.

[108] A. Freundlich and A. Alemu, "Multi quantum well multijunction solar cell for space applications," Phys. Status Solidi C, vol. 2, p. 2978, 2005.

[109] J. G. J. Adams, B. C. Browne, I. M. Ballard, J. P. Connolly, N. L. A. Chan, A. Ioannides, W. Elder, P. N. Stavrinou, K. W. J. Barnham, and N. J. Ekins-Daukes, "Recent results for single-junction and tandem quantum well solar cells," Prog. Photovolt: Res. Appl., vol. 19, no. 7 , pp. 865-877, 2011.

[110] G. K. Bradshaw, J. P. Samberg, C. Z. Carlin, P. C. Colter, K. M. Edmondson, W. Hong, C. Fetzer, N. Karam, and S. M. Bedair, "GaInP/GaAs tandem solar cells with InGaAs/GaAsP multiple quantum wells," IEEE J. Photovolt., vol. 4, pp. 614-619, 2014.

[111] A. Gonzalo, A. D. Utrilla, D. F. Reyes, V. Braza, J. M. Llorens, D. Fuertes Marrn, B. Aln, T. Ben, D. Gonzlez, A. Guzman, A. Hierro, and J. M. Ulloa, "Strainbalanced type-II superlattices for efficient multijunction solar cells," Sci. Rep., vol. 7, p. 4012, 2017.

[112] J. Nelson, M. Paxman, K. W. J. Barnham, J. Roberts, and C. Button, "Steady state carrier escape rates from single quantum wells," IEEE J. Quantum Electron., vol. 29, pp. 1460-1468, 1993.

[113] G. Thucydides, J. M. Barnes, E. Tsui, K. W. J. Barnham, C. C. Phillips, T. S. Cheng, and C. T. Foxon, "Picosecond photoluminescence studies of carrier escape processes in a single quantum well," Semicond. Sci. Technol., vol. 11, no. 3, p. 331, 1996.

[114] G. Thucydides, J. M. Barnes, E. Tsui, K. W. J. Barnham, C. C. Phillips, T. S. Cheng, and C. T. Foxon, "Tailored carrier escape rates in $\mathrm{GaAs} / \mathrm{Al}_{0.3} \mathrm{Ga}_{0.7} \mathrm{As}$ asymmetric double quantum wells," Semicond. Sci. Technol., vol. 12, p. 35, 1997.

[115] A. Zachariou, J. Barnes, K. W. J. Barnham, J. Nelson, E. S. M. Tsui, J. Epler, and M. Pate, "A carrier escape study from InP/InGaAs single quantum well solar cells," J. Appl. Phys., vol. 83, p. 881, 1998.

[116] H. Schneider and K. v. Klitzing, "Thermionic emission and gaussian transport of holes in a $\mathrm{GaAs} / \mathrm{Al}_{x} \mathrm{Ga}_{1-x} \mathrm{As}$ multiple-quantum-well structure," Phys. Rev. B, vol. 38 , p. $6160,1988$.

[117] T. B. Norris, X. J. Song, W. J. Schaff, L. F. Eastman, G. Wicks, and G. A. Mourou, "Tunneling escape time of electrons from a quantum well under the influence of an electric field," Appl. Phys. Lett., vol. 54, no. 1, pp. 60-62, 1989.

[118] A. M. Fox, R. G. Ispasoiu, C. T. Foxon, J. E. Cunningham, and W. Y. Jan, "Carrier escape mechanisms from GaAs/Al $G a_{x}$ As multiple quantum wells in an electric field," Appl. Phys. Lett., vol. 63, p. 2917, 1993.

[119] J. Barnes, E. S. M. Tsui, K. W. J. Barnham, S. C. McFarlane, C. Button, and J. S. Roberts, "Steady state photocurrent and photoluminescence from single quantum wells as a function of temperature and bias," J. Appl. Phys., vol. 81, no. 2, p. 892, 1997.

[120] U. Aeberhard, "Spectral properties of photogenerated carriers in quantum well solar cells," Sol. Energy Mater. Sol. Cells, vol. 94, no. 11, pp. 1897 - 1902, 2010.

[121] U. Aeberhard, "Quantum-kinetic perspective on photovoltaic device operation in nanostructure-based solar cells," J. Mater. Res., vol. 33, no. 4, p. 373386, 2018.

[122] M. P. Lumb, M. K. Yakes, M. González, I. Vurgaftman, C. G. Bailey, R. Hoheisel, and R. J. Walters, "Double quantum-well tunnel junctions with high peak tunnel currents and low absorption for InP multi-junction solar cells," Appl. Phys. Lett., vol. 100, no. 21, p. 213907, 2012 .

[123] U. Aeberhard, "Rigorous simulation of InAlGaAs-InGaAs bulk and quantum well interband tunnel junctions for multi-junction solar cells," in 2013 IEEE 39th Photovoltaic Specialists Conference (PVSC), pp. 01540157, 2013.

[124] M. Luisier and G. Klimeck, "Simulation of nanowire tunneling transistors: From the Wentzel-KramersBrillouin approximation to full-band phonon-assisted tunneling," J. Appl. Phys., vol. 107, no. 8, p. 084507 , 2010 .

[125] U. Aeberhard, R. Vaxenburg, E. Lifshitz, and S. Tomić, "Fluorescence of colloidal PbSe/PbS QDs in NIR luminescent solar concentrators," Phys. Chem. Chem. Phys., vol. 14, pp. 16223-16228, 2012.

[126] K. Barnham, J. L. Marques, J. Hassard, and P. O'Brien, "Quantum-dot concentrator and thermodynamic model for the global redshift," Appl. Phys. Lett., vol. 76, no. 9, pp. 1197-1199, 2000

[127] S. V. Kilina, C. F. Craig, D. S. Kilin, and O. V. Prezhdo, "Ab initio time-domain study of phononassisted relaxation of charge carriers in a pbse quantum dot," J. Phys. Chem. C, vol. 111, no. 12, pp. 4871-4878, 2007.

[128] J. M. An, M. Califano, A. Franceschetti, and A. Zunger, "Excited-state relaxation in PbSe quantum dots," $J$. Chem. Phys., vol. 128, no. 16, p. 164720, 2008.

[129] N. Vukmirovic, Z. Ikonic, D. Indjin, and P. Harrison, "Quantum transport in semiconductor quantum dot superlattices: Electron-phonon resonances and polaron effects," Phys. Rev. B, vol. 76, p. 245313, 2007.

[130] S. Tomic, A. G. Sunderland, and I. J. Bush, "Parallel multi-band $\mathrm{k}$ [middle dot]p code for electronic structure of zinc blend semiconductor quantum dots," J. Mater. Chem., vol. 16, pp. 1963-1972, 2006.

[131] R. Vaxenburg and E. Lifshitz, "Alloy and heterostructure architectures as promising tools for controlling electronic properties of semiconductor quantum dots," Phys. Rev. B, vol. 85, p. 075304, 2012.

[132] J. M. An, A. Franceschetti, S. V. Dudiy, and A. Zunger, "The peculiar electronic structure of PbSe quantum dots," Nano Letters, vol. 6, no. 12, pp. 2728-2735, 2006. 
[133] R. Oshima, A. Takata, and Y. Okada, "Straincompensated InAs/GaNAs quantum dots for use in high-efficiency solar cells," Appl. Phys. Lett., vol. 93, no. 8, p. 083111, 2008

[134] G. Conibeer, M. Green, E.-C. Cho, D. König, Y.-h. Cho, T. Fangsuwannarak, G. Scardera, E. Pink, Y. Huang, T. Puzzer, S. Huang, D. Song, C. Flynn, S. Park, $\mathrm{X}$. Hao, and D. Mansfield, "Silicon quantum dot nanostructures for tandem photovoltaic cells," Thin Solid Films, vol. 516, no. 20, pp. 6748-6756, 2008.

[135] A. Martí, N. Lopez, E. Antolin, E. Canovas, C. Stanley, C. Farmer, L. Cuadra, and A. Luque, "Novel semiconductor solar cell structures: The quantum dot intermediate band solar cell," Thin Solid Films, vol. 511, p. EMRS, July 2006. 